\title{
Trematodes obtained from the thiarid freshwater snail Melanoides tuberculata (Müller, 1774) as vector of human infections in Thailand
}

\author{
Duangduen Krailas ${ }^{1}$, Suluck Namchote ${ }^{1}$, Tunyarut Koonchornboon ${ }^{2}$, Wivitchuta Dechruksa ${ }^{1}$, \\ Dusit Boonmekam ${ }^{1}$
}

1 Department of Biology, Faculty of Science, Silpakorn University, Nakhonpathom 73000, Thailand

2 Department of Anatomy, Pramongkhutklao College of Medicine, Bangkok 10400, Thailand

Corresponding author: Duangduen Krailas (kduang@su.ac.th, kduang@gmail.com)

\begin{abstract}
Received 8 Jan 2014

Accepted 18 Feb 2014

Published 28 March 2014

Academic editor:

Matthias Glaubrecht

\section{Key Words}

Trematoda

Cercariae

human health

shedding

Thiaridae

Cerithioidea

Larval stages of trematodes obtained from the freshwater snail Melanoides tuberculata (Cerithioidea, Thiaridae) as intermediate host were studied by using cercarial emergence and crushing snails. Between December 2004 and September 2009 snails from one hundred twenty locations in Thailand were collected every two months for one year at each sampling site. Counts per unit of time method was used in this study, and the samples of snails were collected every 10 minutes per sampling by five collectors. The cercarial stages were examined using shedding and crushing methods. The infection rate was found to be $18.79 \%$, i.e. 6,019 animals infected in a total of 32,026 . Nine different types in eighteen species of cercariae were categorized, viz. are (1) Parapleurophocercous cercariae: Haplorchis pumilio, Haplorchis taichui, and Stictodora tridactyla; (2) Pleurophocercous cercariae: Centrocestus formosanus; (3) Xiphidiocercariae: Acanthatrium hitaense, Loxogenoides bicolor, and Haematoloechus similis; (4) Megalurous cercariae: Cloacitrema philippinum and Philophthalmus sp.; (5) Furcocercous cercariae: Cardicola alseae, Alaria mustelae, Transversotrema laruei, Apatemon gracilis, and Mesostephanus appendiculatus; (6) Echinostome cercariae: Echinochasmus pelecani; (7) Amphistome cercariae: Gastrothylax crumenifer; (8) Renicolid cercariae: Cercaria caribbea LXVIII; and, (9) Cotylomicrocercous cercariae: Podocotyle (Podocotyle) lepomis.
\end{abstract}

\section{Introduction}

The neglected tropical diseases (NTDs) represent the most common parasitic infections affecting the world's poorest people (Hotez et al. 2007). In addition to their detrimental effects on health, NTDs have a chronic debilitating effect by undermining the physical and cognitive development of individuals residing in areas infested with NTDs, especially for children and women of child-bearing age. An especially deleterious effect has been shown on their educational performance and future economic productivity (Hotez et al. 2007, 2009).

It is important to note in this context that trematodes infecting humans, especially liver fluke and intestinal flukes, are highly prevalent in Southeast Asian countries (Wongratanacheewin et al. 2001; Chai et al. 2005). These infections have a major public health impact. It has been reported that the highest degree of infections with trematodes were discovered in the gastrointestinal tract of humans living in the north region of Thailand (Pungpak et al. 1998, Radomyos et al. 1998) and the most metacercarial species were found in cyprinoid fish in the north and northeastern regions (Srisawangwong et al. 1997b, Sukontason et al. 1999). The liver fluke Opisthorchis viverrini can cause chalangiocarcinoma, a kind of cancer in gall bladder (Sripa et al. 2010), while the intestinal fluke Haplorchis taichui is a possible agent of irritable bowel syndrome-like symptoms (Watthanakulpanich et al. 2010). However, Thai people have considerably underestimated these trematodes by continually eating Thai traditional food prepared from raw freshwater fish (Chuboon et al. 2005). So the prevalence of trematodes in Thailand has been a continual problem until now. 


\section{Life cycle of trematodes}

Trematodes need three hosts to complete their life cycles. After their eggs hatch in the water source such as canals and streams, the miracidium, their first larval stage, will swim and find the first intermediate host, namely freshwater snails and terrestrial snails close to water. In particular, snail species of the genus Bithynia are known as intermediate hosts of the liver fluke Opisthorchis viverrini (Tesana 2002). Some edible mollusks, such as the viviparid Filopaludina spp. and the bivalve Corbicula spp., are known as the first and second intermediate hosts of echinostome intestinal fluke (Temcharoen 1992a, b, Krailas et al. 2008). Thus, we can call these trematode infections mollusk-transmitted diseases.

In the snails, they will develop from miracidium to sporocyst to redia and finally to cercaria. Cercaria will leave the snails, head to the second intermediate host, e.g. freshwater fish, and develop to be metacercaria, the infective stage. At least 18 species of cyprinoid fish act as the second intermediate hosts. If the vertebrates, like animals and humans, eat the infected raw fish, they will receive the metacercaria, which will not be digested by digestive enzymes. Instead, the trematodes will become adult and lay eggs, which are then emitted to the water again with the hosts'stool.

\section{Survey of freshwater snails as the first intermediate hosts in Thailand}

Several studies have been conducted on the fauna of mollusks with focus on their trematode infections (Wegeberg et al. 1999, Abdul-Salam et al. 2004). Not only in Asia but also Africa and Australia, these trematodes have been widely studied (Diaz et al. 2008, Derraik 2008). In Thailand, medically-important freshwater snails have been investigated since 1980 (Upatham et al. 1980, 1981, Nithiuthai et al. 2002, Krailas et al. 2003, Sri-aroon et al. 2005, Ukong et al. 2007, Dechruksa et al. 2007, Krailas et al. 2008). It was found that because of their life cycle and host specificity, the distribution of trematodes depends on the presence of the first and second intermediate hosts, as well as the eating habit of local people (Radomyos et al. 1998).

\section{Freshwater Snails of the Thiaridae in Thailand}

Many of the trematode infected freshwater snails are from the Cerithoidea, a large, essentially marine, group of caenogastropods with approximately 200 genera and with mainly pan-tropical distributions, which have been used also as model for evolutionary systematic studies (Glaubrecht 1996, 1999, 2000, 2011, Glaubrecht et al. 2009). To focus on the family Thiaridae, this group rep- resents, as became evident in recent studies (Glaubrecht 1996, 1999, 2011, Lydeard et al. 2002, Strong et al. 2008, 2011), one of the two (or three) independent invasions into and colonizations of freshwater habitats. Mainly distributed in Southeast Asia, they are to be considered, together with the Pachychilidae as most significant intermediate hosts for infections in humans. For instance, the thiarids Tarebia granifera and Thiara toucheana, as well as the pachychilid Brotia asperata, Brotia costula episcopalis and Brotia c. peninsularis were found to be the first intermediate hosts for lung flukes (Tang 1940, Tubangui et al. 1950, Davis 1971, Brandt 1974). In addition, the thiarids Tarebia granifera and Melanoides tuberculata are the first intermediate hosts for intestinal fluke and blood flukes (Malek and Cheng 1974, Pointier and Jourdane 2000). In Thailand, T. granifera and $M$. tuberculata have been reported as the first intermediate host for lung and intestinal flukes (Upatham et al. 1995, Ukong et al. 2007, Dechruksa et al. 2007).

\section{Melanoides tuberculata (Müller, 1774)}

This taxon is common to freshwaters within its native distributional range that covers much of tropical Africa, Asia and the Oceania. It is now also present in much of the tropical and subtropical New World as a consequence of introductions that started during the last century (Madsen and Frandsen 1989). They were described as alien species around the world. Moreover, the species exhibits considerable polymorphism in shell ornamentation across its geographical range; however, at the same time among sites discrete lineages or 'morphs' of M. tuberculata can be separated by shell characters, such as coloration and ornamentation, apparently due to the predominantly parthenogenetic reproduction resulting from negligible intrapopulation variability in these traits (Samadi et al. 1999). For example, on Martinique Islands, each morph of $M$. tuberculata is different in terms of juveniles, growth rate and even parasite infection rate.

M. tuberculata is considered to be of medical significance, as most of the above cited parasites can affect humans. Although there can be considerable seasonal variation in the intensity of parasitism in these snails, the incidence of $M$. tuberculata with trematode parasites has been recorded to be as high as $92 \%$ (Derraik 2008). A checklist from 136 scientific published studies revealed that $M$. tuberculata could be host for flukes, identified as belonging to 17 families, 25 genera, and 37 species (Pinto and De Melo 2011). These trematodes are both animals and human parasites. Nevertheless, in Thailand there are only very few reports about Melanoides infection in certain specific areas of the country to date. However, investigations around Thailand have never been conducted. So, in the present study we have surveyed now the trematode infections of M. tuberculata all over Thailand. 


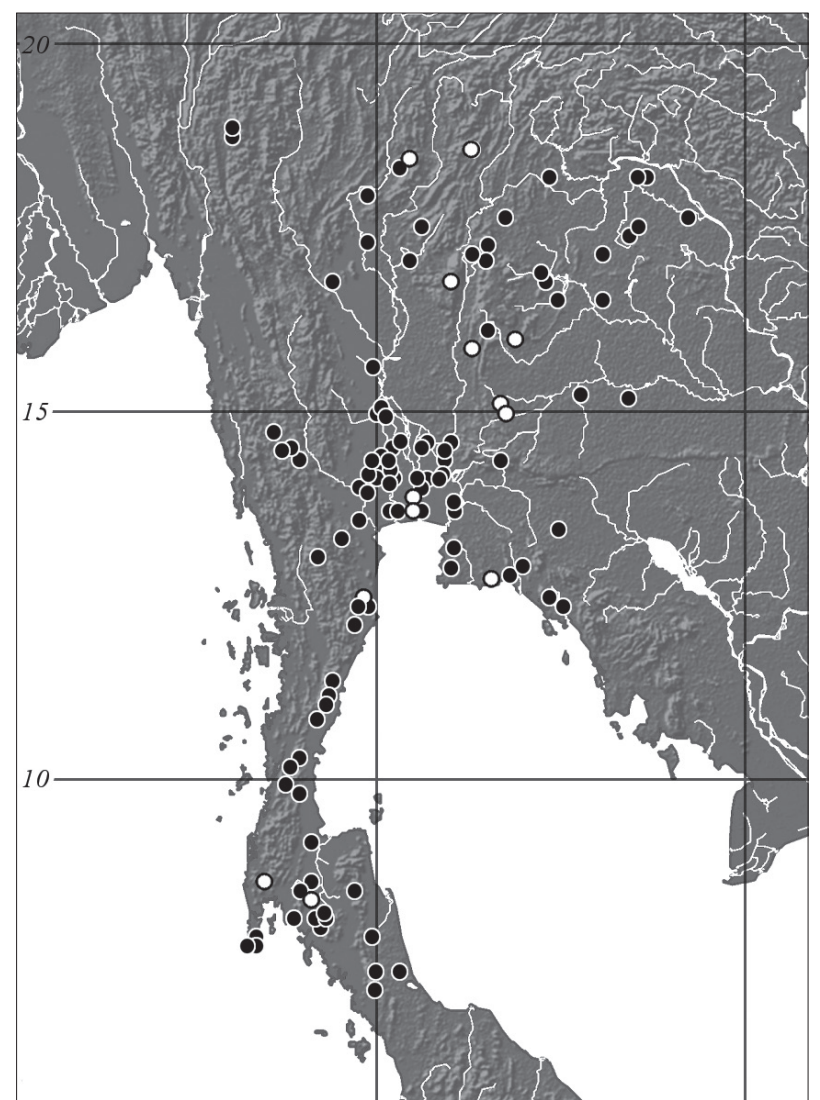

Figure 1. Occurrence of Melanoides tuberculata in Thailand. Closed symbol - M. tuberculata with infection; open symbol M. tuberculata without infection.

\section{Materials and methods}

\section{Sampling sites}

One hundred twenty locations in Thailand, which are used by humans as sources of water, were examined (snail collection sites). For the exact data, please refer to the first section of the Result section in this report. The positions of collection sites were recorded by GPS (Garmin PLUS III, Taiwan). The localities of the relevant samples were mapped on a dot-by-dot basis to a digitally reduced version of the drainage pattern map of Thailand, as developed in Dechruksa et al. (2013). This map was created using a Reliefkarte on the basis of the Global 30-Arc-Second Elevation Data (GTOPO30) from the U.S. Geological Survey and a river map from the Map/server Aquarius Geomar, and then compiled using Adobe Photoshop CS3 and Adobe Illustrator.

\section{Collection of snails}

Between December 2004 and September 2009, snails were collected every two months for one year from each localities, using the counts per unit of time sampling method (Olivier and Schneiderman 1956). Five researchers collected samples by handpicking and scooping ev- ery 10 minutes at each sampling site. The snail samples were then categorized into species according to their shell morphology. They were later examined for trematode infections.

\section{Examination for parasitic infections}

Parasitic infections were investigated by using snail shedding and crushing methods. Emerged cercariae were collected in dechlorinated water and observed for their swimming behavior (Krailas et al. 2003). Sporocysts and rediae were examined under a dissecting microscope.

\section{Study of cercarial morphology}

The cercariae were studied unstained or vitally stained with $0.5 \%$ neutral red and Semichon's acetic carmine \& fast green. Descriptions of the morphology and anatomy of cercariae were based on the study of living cercariae that had escaped from the snails. Sample measurements (average size) in micrometers were taken from 20 specimens fixed in 10\% formalin. Measurements in micrometers with averages in parentheses were taken from 20 specimens. Details of the cercariae were drawn using a camera lucida, and all their species were identified (Schell 1962, 1970, Nasir 1974, Yamaguti 1975; Ito 1980). For scanning electron microscopy, cercariae were fixed in $2.5 \%$ glutaraldehyde phosphate buffer $(0.1 \mathrm{~mol} / 1$, ph 7.4) at $4{ }^{\circ} \mathrm{C}$ for at least 2 hours and post-fixed in $1 \%$ osmium tetroxide in the same buffer for 2 hours at $4{ }^{\circ} \mathrm{C}$. They were dehydrated through a graded series of ethanol, and then dried in a critical point dryer using liquid carbon dioxide as a transition medium. The specimens were coated with gold-palladium in an ion-sputtering apparatus (Polaron CPD 7501, UK), and then examined in a scanning electron microscope (Camscan mx 2000, UK or JEOL, JSM-5410 LV, Japan).

\section{Results}

\section{Melanoides tuberculata in Thailand}

The thiarid Melanoides tuberculata was found in and sampled from study sites in five regions in Thailand, as shown in Table 1, Fig. 1. These include the following:

The North: N1 = Sakunotayan Waterfall, Wangtong District, Phitsanulok Province (SUT0109001) (N 1650'20.6”, E 100³2'15.6”, Altitude 40 m); N2 = Kaeng Sopha Waterfall, Wangtong District, Phitsanulok Province (SUT0109002) (N 1652'22.3”, E 10050'29.6”, Altitude 398 m); N3 = Thung Salaeng Luang Stream, Wangtong District, Phitsanulok Province (SUT0109003) (N 1650'50.0”, E 10051'57.2”, Altitude $452 \mathrm{~m})$; N4 = Pha Laht Waterfall, Nakhonthai District, Phitsanulok Province (SUT0109004) (N 1701'69.1”, E 10056'77.8', Altitude $267 \mathrm{~m}$ ); N5 = Thum Pla Stream, Muang District, Mae Hong Son Province (SUT0109005) 
Table 1. Locations in Thailand with Melanoides tuberculata found, the number of collected snails and infection rates.

\begin{tabular}{|c|c|c|c|c|c|}
\hline $\begin{array}{l}\text { No. } \\
\text { (no. specimen) }\end{array}$ & Name & GPS & \begin{tabular}{|c|}
$\begin{array}{c}\text { No. of } \\
\text { collected } \\
\text { snails }\end{array}$ \\
\end{tabular} & $\begin{array}{c}\text { No. of } \\
\text { Infected } \\
\text { snails }\end{array}$ & $\begin{array}{c}\text { Infection } \\
\text { rates } \\
(\%)\end{array}$ \\
\hline \multicolumn{6}{|l|}{ North } \\
\hline $\begin{array}{l}\text { N1 } \\
\text { (SUT0109001) }\end{array}$ & $\begin{array}{l}\text { Sakunotayan Waterfall, Wangtong District, } \\
\text { Phitsanulok }\end{array}$ & $\begin{array}{l}\text { N } 16^{\circ} 50^{\prime} 20.6^{\prime \prime} \text { E } 100^{\circ} 32^{\prime} \\
15.6^{\prime \prime} \text { Altitude } 40 \mathrm{~m}\end{array}$ & 13 & 3 & 23.08 \\
\hline $\begin{array}{l}\text { N2 } \\
\text { (SUT0109002) }\end{array}$ & $\begin{array}{l}\text { Kaeng Sopha Waterfall, Wangtong District, } \\
\text { Phitsanulok }\end{array}$ & $\begin{array}{l}\text { N } 16^{\circ} 52^{\prime} 22.3^{\prime \prime} \text { E } 100^{\circ} 50^{\prime} \\
29.6^{\prime \prime} \text { Altitude } 398 \text { m }\end{array}$ & 3 & 1 & 33.33 \\
\hline $\begin{array}{l}\text { N3 } \\
\text { (SUT0109003) }\end{array}$ & $\begin{array}{l}\text { Thung Salaeng Luang Stream, Wangtong Dis- } \\
\text { trict, Phitsanulok }\end{array}$ & $\begin{array}{l}\mathrm{N} 16^{\circ} 50^{\prime} 50.0^{\prime \prime} \mathrm{E} 100^{\circ} 51^{\prime} \\
57.2^{\prime \prime} \text { Altitude } 452 \mathrm{~m}\end{array}$ & 3 & 0 & 0 \\
\hline $\begin{array}{l}\text { N4 } \\
\text { (SUT0109004) }\end{array}$ & $\begin{array}{l}\text { Pha Laht Waterfall, Nakhonthai District, Phitsa- } \\
\text { nulok }\end{array}$ & $\begin{array}{l}\mathrm{N} 17^{\circ} 01^{\prime} 69.1^{\prime \prime} \mathrm{E} 100^{\circ} 56^{\prime} \\
77.8^{\prime \prime} \text { Altitude } 267 \mathrm{~m}\end{array}$ & 5 & 4 & 80 \\
\hline $\begin{array}{l}\text { N5 } \\
\text { (SUT0109005) }\end{array}$ & $\begin{array}{l}\text { Thum Pla Stream, Muang District, Mae Hong } \\
\text { Son }\end{array}$ & $\begin{array}{l}\text { N 19² 25' 31.7" E } 97^{\circ} 59^{\prime} \\
\text { 24.9" Altitude } 343 \text { m }\end{array}$ & 60 & 38 & 63.33 \\
\hline $\begin{array}{l}\text { N6 } \\
\text { (SUT0109006) }\end{array}$ & $\begin{array}{l}\text { Huay Sua Thao Stream, Muang District, Mae } \\
\text { Hong Son }\end{array}$ & $\begin{array}{l}\mathrm{N} 19^{\circ} 15^{\prime} 32.0^{\prime \prime} \mathrm{E} 97^{\circ} 54^{\prime} \\
43.7^{\prime \prime} \text { Altitude } 237 \mathrm{~m}\end{array}$ & 51 & 9 & 17.65 \\
\hline $\begin{array}{l}\text { N7 } \\
\text { (SUT0109007) }\end{array}$ & $\begin{array}{l}\text { Klong Nam Lai Waterfall, Klong Lan District, } \\
\text { Kam Phaeng Phet }\end{array}$ & $\begin{array}{l}\text { N } 16^{\circ} 11^{\prime} 32.7^{\prime \prime} \text { E } 99^{\circ} 15^{\prime} \\
51.0^{\prime \prime} \text { Altitude } 241 \mathrm{~m}\end{array}$ & 63 & 28 & 44.44 \\
\hline $\begin{array}{l}\text { N8 } \\
\text { (SUT0109008) }\end{array}$ & $\begin{array}{l}\text { Tad Duen Waterfall, Sri Satchanalai District, } \\
\text { Sukhothai }\end{array}$ & $\begin{array}{l}\left(\mathrm{N} 17^{\circ} 33^{\prime} 23.2^{\prime \prime} \text { E } 99^{\circ} 29^{\prime}\right. \\
\left.76.8^{\prime \prime} \text { Altitude } 414 \mathrm{~m}\right)\end{array}$ & 161 & 38 & 23.60 \\
\hline $\begin{array}{l}\text { N9 } \\
\text { (SUT0109009) }\end{array}$ & $\begin{array}{l}\text { Sri Satchanalai Stream, Sri Satchanalai National } \\
\text { Park, Sukhothai }\end{array}$ & $\begin{array}{l}\text { N } 17^{\circ} 33^{\prime} 5.9^{\prime \prime} \text { E } 99^{\circ} 29^{\prime} 24.8^{\prime \prime} \\
\text { Altitude } 182 \mathrm{~m}\end{array}$ & 113 & 24 & 21.24 \\
\hline $\begin{array}{l}\text { N10 } \\
\text { (SUT0109010) }\end{array}$ & Mae Pool Waterfall, Lub Lae District, Ut-taradit & $\begin{array}{l}\mathrm{N} 17^{\circ} 43^{\prime} 45.0^{\prime \prime} \mathrm{E} 99^{\circ} 58^{\prime} \\
50.6^{\prime \prime} \text { Altitude } 164 \mathrm{~m}\end{array}$ & 70 & 9 & 12.86 \\
\hline $\begin{array}{l}\text { N11 } \\
\text { (SUT0109011) }\end{array}$ & Chueng Thong Waterfall, Muang District, Prae & $\begin{array}{l}\mathrm{N} 18^{\circ} 01^{\prime} 54.2^{\prime \prime} \mathrm{E} 100^{\circ} 15^{\prime} \\
52.8^{\prime \prime} \text { Altitude } 298 \mathrm{~m}\end{array}$ & 7 & 3 & 42.85 \\
\hline $\begin{array}{l}\text { N12 } \\
\text { (SUT0109012) }\end{array}$ & Huay Sa Nien Stream, Muang district, Nan & $\begin{array}{l}\mathrm{N} 18^{\circ} 51^{\prime} 1.3^{\prime \prime} \mathrm{E} 100^{\circ} 39^{\prime} \\
16.2^{\prime \prime} \text { Altitude } 280 \mathrm{~m}\end{array}$ & 60 & 0 & 0 \\
\hline $\begin{array}{l}\text { N13 } \\
\text { (SUT0109013) }\end{array}$ & $\begin{array}{l}\text { Huay Ton Phueng Waterfall, Doi Pu Nang Nation- } \\
\text { al Park, Chiang Muon District, Phyao }\end{array}$ & $\begin{array}{l}\mathrm{N} 18^{\circ} 55^{\prime} 5.3^{\prime \prime} \mathrm{E} 100^{\circ} 12^{\prime} \\
15.7^{\prime \prime} \text { Altitude } 379 \mathrm{~m}\end{array}$ & 131 & 1 & 0.76 \\
\hline $\begin{array}{l}\text { N14 } \\
\text { (SUT0109014) }\end{array}$ & $\begin{array}{l}\text { Tansawan Waterfall, Doi Pu Nang National Park, } \\
\text { Chiang Muon District, Phyao }\end{array}$ & $\begin{array}{l}\mathrm{N} 18^{\circ} 51^{\prime} 22.7^{\prime \prime} \mathrm{E} 100^{\circ} 11^{\prime} \\
9.6^{\prime \prime} \text { Altitude } 420 \mathrm{~m}\end{array}$ & 368 & 16 & 4.35 \\
\hline $\begin{array}{l}\text { N15 } \\
\text { (SUT0109015) }\end{array}$ & Mae Mine Stream, Mae Ta District, Lampang & $\begin{array}{l}\mathrm{N} 18^{\circ} 07^{\prime} 1.8^{\prime \prime} \mathrm{E} 99^{\circ} 37^{\prime} 35.1^{\prime \prime} \\
\text { Altitude } 269 \mathrm{~m}\end{array}$ & 201 & 5 & 2.49 \\
\hline \multicolumn{6}{|l|}{ Northeast } \\
\hline $\begin{array}{l}\text { NE16 } \\
\text { (SUT0109016) }\end{array}$ & $\begin{array}{l}\text { Huay Lum Po Dang Stream, Thepsathit District, } \\
\text { Chaiyapoom }\end{array}$ & $\begin{array}{l}\mathrm{N} 15^{\circ} 33^{\prime} 42.8^{\prime \prime} \mathrm{E} 101^{\circ} 24^{\prime} \\
56.9^{\prime \prime} \text { Altitude } 471 \mathrm{~m}\end{array}$ & 311 & 0 & 0 \\
\hline $\begin{array}{l}\text { NE17 } \\
\text { (SUT0109017) }\end{array}$ & $\begin{array}{l}\text { Sai Thong Waterfall, Sai Thong National Park, } \\
\text { Nong Bua Ra Hoew District, Chaiyapoom }\end{array}$ & $\begin{array}{l}\mathrm{N} 15^{\circ} 52^{\prime} 34.7^{\prime \prime} \mathrm{E} 101^{\circ} 30^{\prime} \\
34.7^{\prime \prime} \text { Altitude } 397 \mathrm{~m}\end{array}$ & 275 & 14 & 5.09 \\
\hline $\begin{array}{l}\text { NE18 } \\
\text { (SUT0109018) }\end{array}$ & Tad Tone Waterfall, Muang District, Chaiyaphom & $\begin{array}{l}\mathrm{N} 15^{\circ} 58^{\prime} 42.5^{\prime \prime} \mathrm{E} 102^{\circ} 02^{\prime} \\
\text { 24.9" Altitude } 384 \mathrm{~m}\end{array}$ & 70 & 0 & 0 \\
\hline $\begin{array}{l}\text { NE19 } \\
\text { (SUT0109019) }\end{array}$ & $\begin{array}{l}\text { Kongkaew Waterfall, Khao Yai National Park, Pak } \\
\text { Chong District, Nakhon Ratchasima }\end{array}$ & $\begin{array}{l}\mathrm{N} 14^{\circ} 26^{\prime} 14.8^{\prime \prime} \mathrm{E} 101^{\circ} 22^{\prime} \\
37.6^{\prime \prime} \text { Altitude } 713 \mathrm{~m}\end{array}$ & 15 & 0 & 0 \\
\hline $\begin{array}{l}\text { NE20 } \\
\text { (SUT0109020) }\end{array}$ & $\begin{array}{l}\text { Lam Takhong Stream, Khao Yai National Park, } \\
\text { Pak Chong district, Nakhon Ratchasima }\end{array}$ & $\begin{array}{l}\mathrm{N} 14^{\circ} 25^{\prime} 19.6^{\prime \prime} \mathrm{E} 101^{\circ} 23^{\prime} \\
26.3^{\prime \prime} \text { Altitude } 700 \mathrm{~m}\end{array}$ & 344 & 0 & 0 \\
\hline $\begin{array}{l}\text { NE21 } \\
\text { (SUT0109021) }\end{array}$ & Ban Cha Rut Reservoir, Bua Ched District, Surin & $\begin{array}{l}\mathrm{N} 14^{\circ} 25^{\prime} 50.4^{\prime \prime} \mathrm{E} 103^{\circ} 57^{\prime} \\
47.7^{\prime \prime} \text { Altitude } 201 \mathrm{~m}\end{array}$ & 441 & 150 & 34.01 \\
\hline $\begin{array}{l}\text { NE22 } \\
\text { (SUT0109022) }\end{array}$ & $\begin{array}{l}\text { Nong Bua Rai Reservoir, Khao Panomroong, Pra } \\
\text { Kone Chai District, Burirum }\end{array}$ & $\begin{array}{l}\mathrm{N} 14^{\circ} 32^{\prime} 51.2^{\prime \prime} \mathrm{E} 102^{\circ} 58^{\prime} \\
9.4^{\prime \prime} \text { Altitude } 202 \mathrm{~m}\end{array}$ & 48 & 22 & 45.83 \\
\hline $\begin{array}{l}\text { NE23 } \\
\text { (SUT0109023) }\end{array}$ & Pla Ba Waterfall, Pu Rua District, Loei & $\begin{array}{l}\mathrm{N} 17^{\circ} 23^{\prime} 24.3^{\prime \prime} \mathrm{E} 101^{\circ} 22^{\prime} \\
27.4^{\prime \prime} \text { Altitude } 640 \mathrm{~m}\end{array}$ & 19 & 0 & 0 \\
\hline $\begin{array}{l}\text { NE24 } \\
\text { (SUT0109024) }\end{array}$ & $\begin{array}{l}\text { Than Thong Waterfall, Sung Kom District, Nong } \\
\text { Khai }\end{array}$ & $\begin{array}{l}\mathrm{N} 18^{\circ} 01^{\prime} 34.7^{\prime \prime} \mathrm{E} 102^{\circ} 22^{\prime} \\
8.7^{\prime \prime} \text { Altitude } 195 \mathrm{~m}\end{array}$ & 678 & 102 & 15.04 \\
\hline $\begin{array}{l}\text { NE25 } \\
\text { (SUT0109025) }\end{array}$ & $\begin{array}{l}\text { Huay Hor Water Gate, Muang District, Nakhon } \\
\text { Panom }\end{array}$ & $\begin{array}{l}\text { N } 17^{\circ} 21^{\prime} 8.4^{\prime \prime} \text { E } 104^{\circ} 47^{\prime} 2.1^{\prime \prime} \\
\text { Altitude } 145 \mathrm{~m}\end{array}$ & 215 & 70 & 32.56 \\
\hline $\begin{array}{l}\text { NE26 } \\
\text { (SUT0109026) }\end{array}$ & $\begin{array}{l}\text { Tad Kham Waterfall, Pu Lung Ga National Park, } \\
\text { Ban Pang District, Nakhon Panom }\end{array}$ & $\begin{array}{l}\text { N } 17^{\circ} 57^{\prime} 1.4^{\prime \prime} \text { E } 104^{\circ} 09^{\prime} \\
39.9^{\prime \prime} \text { Altitude } 148 \mathrm{~m}\end{array}$ & 1,257 & 887 & 70.56 \\
\hline $\begin{array}{l}\text { NE27 } \\
\text { (SUT0109027) }\end{array}$ & $\begin{array}{l}\text { Tad Po waterfall, Pu Lung Ga National Park, Ban } \\
\text { Pang district, Nakhon Panom }\end{array}$ & $\begin{array}{l}\mathrm{N} 17^{\circ} 59^{\prime} 0.9^{\prime \prime} \mathrm{E} 104^{\circ} 08^{\prime} \\
34.3^{\prime \prime} \text { Altitude } 148 \mathrm{~m}\end{array}$ & 654 & 167 & 25.54 \\
\hline
\end{tabular}




\begin{tabular}{|c|c|c|c|c|c|}
\hline $\begin{array}{c}\text { No. } \\
\text { (no. specimen) }\end{array}$ & Name & GPS & \begin{tabular}{|c|}
$\begin{array}{c}\text { No. of } \\
\text { collected } \\
\text { snails }\end{array}$ \\
\end{tabular} & \begin{tabular}{|c|} 
No. of \\
Infected \\
snails \\
\end{tabular} & $\begin{array}{c}\text { Infection } \\
\text { rates } \\
(\%)\end{array}$ \\
\hline $\begin{array}{l}\text { NE28 } \\
\text { (SUT0109028) }\end{array}$ & Nong Haan, Muang Distrcit, Sakol Nakhon & $\begin{array}{l}\mathrm{N} 17^{\circ} 09^{\prime} 50.1^{\prime \prime} \mathrm{E} 104^{\circ} 09^{\prime} \\
\text { 43.7" Altitude } 161 \mathrm{~m}\end{array}$ & 68 & 3 & 4.41 \\
\hline $\begin{array}{l}\text { NE29 } \\
\text { (SUT0109029) }\end{array}$ & $\begin{array}{l}\text { Nam Poong Dam, Gud Bak Distrcit, Sakol } \\
\text { Nakhon }\end{array}$ & $\begin{array}{l}\mathrm{N} 16^{\circ} 58^{\prime} 11.8^{\prime \prime} \mathrm{E} 103^{\circ} 59^{\prime} \\
\text { 13.4" Altitude } 290 \mathrm{~m}\end{array}$ & 260 & 57 & 21.92 \\
\hline $\begin{array}{l}\text { NE30 } \\
\text { (SUT0109030) }\end{array}$ & Lam Pow Dam, Muang District, Sri Sa Ket & $\begin{array}{l}\mathrm{N} 16^{\circ} 36^{\prime} 22.3^{\prime \prime} \mathrm{E} 103^{\circ} 26^{\prime} \\
\text { 27.5" Altitude } 165 \mathrm{~m}\end{array}$ & 329 & 9 & 2.74 \\
\hline $\begin{array}{l}\text { NE31 } \\
\text { (SUT0109031) }\end{array}$ & $\begin{array}{l}\text { Ban Nong Wang Wong Reservior, Sri Som Det } \\
\text { District, Roi-Et }\end{array}$ & $\begin{array}{l}\mathrm{N} 15^{\circ} 56^{\prime} 53.5^{\prime \prime} \mathrm{E} 103^{\circ} 31^{\prime} \\
27.8^{\prime \prime} \text { Altitude } 178 \mathrm{~m}\end{array}$ & 1,281 & 126 & 9.84 \\
\hline $\begin{array}{l}\text { NE32 } \\
\text { (SUT0109032) }\end{array}$ & Bung Toong Sang, Muang District, Khon Kaen & $\begin{array}{l}\mathrm{N} 16^{\circ} 26^{\prime} 27.8^{\prime \prime} \mathrm{E} 102^{\circ} 51^{\prime} \\
28.1^{\prime \prime} \text { Altitude } 154 \mathrm{~m}\end{array}$ & 173 & 3 & 1.73 \\
\hline $\begin{array}{l}\text { NE33 } \\
\text { (SUT0109033) }\end{array}$ & Bung Kaen Nakhon, Muang District, Khon Kaen & $\begin{array}{l}\mathrm{N} 16^{\circ} 24^{\prime} 46.6^{\prime \prime} \text { E } 102^{\circ} 50^{\prime} \\
21.9^{\prime \prime} \text { Altitude } 143 \mathrm{~m}\end{array}$ & 218 & 18 & 8.26 \\
\hline $\begin{array}{l}\text { NE34 } \\
\text { (SUT0109034) }\end{array}$ & $\begin{array}{l}\text { Nong Sa Ad Bamroong Reservoir, Kosum Pisai } \\
\text { District, Mahasarkham }\end{array}$ & $\begin{array}{l}\mathrm{N} 16^{\circ} 18^{\prime} 0.5^{\prime \prime} \mathrm{E} 102^{\circ} 54^{\prime} \\
38.5^{\prime \prime} \text { Altitude } 169 \mathrm{~m}\end{array}$ & 552 & 31 & 5.62 \\
\hline $\begin{array}{l}\text { NE35 } \\
\text { (SUT0109035) }\end{array}$ & $\begin{array}{l}\text { Tad Tone Waterfall, Nong Soong District, Muk- } \\
\text { daharn }\end{array}$ & $\begin{array}{l}\text { N } 16^{\circ} 29^{\prime} 34.9^{\prime \prime} \text { E } 104^{\circ} 19^{\prime} \\
1.1^{\prime \prime} \text { Altitude } 219 \mathrm{~m}\end{array}$ & 427 & 81 & 18.97 \\
\hline \multicolumn{6}{|c|}{ I } \\
\hline $\begin{array}{l}\text { E36 } \\
\text { (SUT0109036) }\end{array}$ & $\begin{array}{l}\text { Khao Khaew National Park, Sriracha District, } \\
\text { Chonburi }\end{array}$ & $\begin{array}{l}\mathrm{N} 13^{\circ} 12^{\prime} 45.0^{\prime \prime} \mathrm{E} 101^{\circ} 03^{\prime} \\
50.2^{\prime \prime} \text { Altitude } 128 \mathrm{~m}\end{array}$ & 613 & 93 & 15.17 \\
\hline $\begin{array}{l}\text { E37 } \\
\text { (SUT0109037) }\end{array}$ & $\begin{array}{l}\text { Ban Nong Pla Lai, Bang lamung District, Chon- } \\
\text { buri }\end{array}$ & $\begin{array}{l}\mathrm{N} 12^{\circ} 57^{\prime} 54.3^{\prime \prime} \text { E } 100^{\circ} 56^{\prime} \\
47.8^{\prime \prime} \text { Altitude } 17 \mathrm{~m}\end{array}$ & 610 & 80 & 13.11 \\
\hline $\begin{array}{l}\text { E38 } \\
\text { (SUT0109038) }\end{array}$ & Rayong River, Muang District, Rayong & $\begin{array}{l}\mathrm{N} 12^{\circ} 39^{\prime} 52.6^{\prime \prime} \mathrm{E} 101^{\circ} 14^{\prime} \\
\text { 48.5" Altitude } 6 \mathrm{~m}\end{array}$ & 51 & 0 & 0 \\
\hline $\begin{array}{l}\text { E39 } \\
\text { (SUT0109039) }\end{array}$ & Hin Khao Canal, Muang District, Rayong & $\begin{array}{l}\mathrm{N} 12^{\circ} 36^{\prime} 31.7^{\prime \prime} \mathrm{E} 101^{\circ} 23^{\prime} \\
\text { 22.4" Altitude } 1 \mathrm{~m}\end{array}$ & 252 & 2 & 0.79 \\
\hline $\begin{array}{l}\text { E40 } \\
\text { (SUT0109040) }\end{array}$ & Pung rad Canal, Klang District, Rayong & $\begin{array}{l}\mathrm{N} 12^{\circ} 42^{\prime} 49.5^{\prime \prime} \mathrm{E} 101^{\circ} 46^{\prime} \\
\text { 23.4" Altitude } 15 \mathrm{~m}\end{array}$ & 827 & 42 & 5.08 \\
\hline $\begin{array}{l}\text { E41 } \\
\text { (SUT0109041) }\end{array}$ & Chantaburi River, Muang District, Chantaburi & $\begin{array}{l}\mathrm{N} 12^{\circ} 36^{\prime} 13.8^{\prime \prime} \text { E } 102^{\circ} 07^{\prime} \\
\text { 11.6" Altitude } 8 \mathrm{~m}\end{array}$ & 278 & 18 & 6.47 \\
\hline $\begin{array}{l}\text { E42 } \\
\text { (SUT0109042) }\end{array}$ & Nam Tok Plew Stream, Plew District, Chantaburi & $\begin{array}{l}\mathrm{N} 12^{\circ} 31^{\prime} 14.3^{\prime \prime} \mathrm{E} 102^{\circ} 10^{\prime} \\
35.4^{\prime \prime} \text { Altitude } 39 \mathrm{~m}\end{array}$ & 223 & 14 & 6.28 \\
\hline $\begin{array}{l}\text { E43 } \\
\text { (SUT0109043) }\end{array}$ & Pa Tong Canal, Soi Dao District, Chantaburi & $\begin{array}{l}\text { N } 13^{\circ} 07^{\prime} 5.9^{\prime \prime} \text { E } 102^{\circ} 13^{\prime} \\
13.6^{\prime \prime} \text { Altitude } 231 \mathrm{~m}\end{array}$ & 206 & 20 & 9.71 \\
\hline $\begin{array}{l}\text { E44 } \\
\text { (SUT0109044) }\end{array}$ & Klong Kaew Waterfall, Bo Rai District, Trad & $\begin{array}{l}\mathrm{N} 12^{\circ} 37^{\prime} 3.0^{\prime \prime} \mathrm{E} 102^{\circ} 34^{\prime} \\
52.0^{\prime \prime} \text { Altitude } 81 \mathrm{~m}\end{array}$ & 347 & 83 & 23.92 \\
\hline $\begin{array}{l}\text { E45 } \\
\text { (SUT0109045) }\end{array}$ & Sra Kaew, Muang District, Sra Kaew & $\begin{array}{l}\mathrm{N} 13^{\circ} 49^{\prime} 7.0^{\prime \prime} \mathrm{E} 102^{\circ} 03^{\prime} \\
\text { 37.9" Altitude } 43 \mathrm{~m}\end{array}$ & 480 & 188 & 39.17 \\
\hline $\begin{array}{l}\text { E46 } \\
\text { (SUT0109046) }\end{array}$ & Eto Waterfall, Muang District, Prachinburi & $\begin{array}{l}\text { N 14 } 08^{\prime} 58.9^{\prime \prime} \text { E } 101^{\circ} 24^{\prime} \\
\text { 45.4" Altitude } 39 \mathrm{~m}\end{array}$ & 810 & 317 & 39.14 \\
\hline \multicolumn{6}{|l|}{ Central } \\
\hline $\begin{array}{l}\text { C47 } \\
\text { (SUT0109047) }\end{array}$ & Dusit Zoo Pond, Dusit, Bangkok & $\begin{array}{l}\mathrm{N} 13^{\circ} 46^{\prime} 17.4^{\prime \prime} \mathrm{E} 100^{\circ} 31^{\prime} \\
14.8^{\prime \prime} \text { Altitude } 2 \mathrm{~m}\end{array}$ & 26 & 9 & 34.62 \\
\hline $\begin{array}{l}\text { C48 } \\
\text { (SUT0109048) }\end{array}$ & $\begin{array}{l}\text { Drainage at Kasetsart University, Bang Khen } \\
\text { campus, Bangkok }\end{array}$ & $\begin{array}{l}\mathrm{N} 13^{\circ} 50^{\prime} 40.7^{\prime \prime} \mathrm{E} 100^{\circ} 34^{\prime} \\
33.5^{\prime \prime} \text { Altitude } 5 \mathrm{~m}\end{array}$ & 199 & 5 & 2.51 \\
\hline $\begin{array}{l}\text { C49 } \\
\text { (SUT0109049) }\end{array}$ & $\begin{array}{l}\text { Pond at Kasetsart University, Bang Khen Cam- } \\
\text { pus, Bangkok }\end{array}$ & $\begin{array}{l}\mathrm{N} 13^{\circ} 50^{\prime} 22.6^{\prime \prime} \mathrm{E} 100^{\circ} 34^{\prime} \\
43.4^{\prime \prime} \text { Altitude } 1 \mathrm{~m}\end{array}$ & 92 & 1 & 1.09 \\
\hline $\begin{array}{l}\text { C50 } \\
\text { (SUT0109050) }\end{array}$ & $\begin{array}{l}\text { Hin Dad Waterfall, Thong Pa Poom District, } \\
\text { Kanchanaburi }\end{array}$ & $\begin{array}{l}\text { N 14 37' 29.8" E } 98^{\circ} 43^{\prime} \\
40.2^{\prime \prime} \text { Altitude } 186 \text { m }\end{array}$ & 13 & 0 & 0 \\
\hline $\begin{array}{l}\text { C51 } \\
\text { (SUT0109051) }\end{array}$ & $\begin{array}{l}\text { Pha Tad Waterfall, Sri Sa wat District, Kan- } \\
\text { chanaburi }\end{array}$ & $\begin{array}{l}\text { N 143' 54.9" E } 98^{\circ} 46^{\prime} \\
41.6^{\prime \prime} \text { Altitude } 196 \text { m }\end{array}$ & 14 & 2 & 14.29 \\
\hline $\begin{array}{l}\text { C52 } \\
\text { (SUT0109052) }\end{array}$ & $\begin{array}{l}\text { Sai Yok Noi Waterfall, Sai Yok District, Kan- } \\
\text { chanaburi }\end{array}$ & $\begin{array}{l}\text { N 14 14' 27.6" E 99 03' } \\
55.9^{\prime \prime} \text { Altitude } 166 \text { m }\end{array}$ & 158 & 35 & 22.15 \\
\hline $\begin{array}{l}\text { C53 } \\
\text { (SUT0109053) }\end{array}$ & $\begin{array}{l}\text { Sai Yok Yai Waterfall, Sai Yok District, Kan- } \\
\text { chanaburi }\end{array}$ & $\begin{array}{l}\mathrm{N} 14^{\circ} 26^{\prime} 03.0^{\prime \prime} \mathrm{E} 98^{\circ} 51^{\prime} \\
\text { 14.7" Altitude } 140 \mathrm{~m}\end{array}$ & 91 & 3 & 3.30 \\
\hline $\begin{array}{l}\text { C54 } \\
\text { (SUT0109054) }\end{array}$ & $\begin{array}{l}\text { Wans Soong Canal, Bang Kla District, Cha- } \\
\text { chuengsao }\end{array}$ & $\begin{array}{l}\mathrm{N} 13^{\circ} 39^{\prime} 46.2^{\prime \prime} \mathrm{E} 101^{\circ} 10^{\prime} \\
48.2^{\prime \prime} \text { Altitude } 18 \mathrm{~m}\end{array}$ & 161 & 19 & 11.80 \\
\hline
\end{tabular}




\begin{tabular}{|c|c|c|c|c|c|}
\hline $\begin{array}{c}\text { No. } \\
\text { (no. specimen) }\end{array}$ & Name & GPS & \begin{tabular}{|c|}
$\begin{array}{c}\text { No. of } \\
\text { collected } \\
\text { snails }\end{array}$ \\
\end{tabular} & \begin{tabular}{|c|}
$\begin{array}{c}\text { No. of } \\
\text { Infected } \\
\text { snails }\end{array}$ \\
\end{tabular} & $\begin{array}{c}\text { Infection } \\
\text { rates } \\
(\%)\end{array}$ \\
\hline $\begin{array}{l}\text { C55 } \\
\text { (SUT0109055) }\end{array}$ & $\begin{array}{l}\text { Sua Noi Canal, Bnag pa Kong District, Cha- } \\
\text { chuengsao }\end{array}$ & $\begin{array}{l}\mathrm{N} 13^{\circ} 34^{\prime} 31.0^{\prime \prime} \mathrm{E} 100^{\circ} 57^{\prime} \\
13.8^{\prime \prime} \text { Altitude } 2 \mathrm{~m}\end{array}$ & 239 & 8 & 3.35 \\
\hline $\begin{array}{l}\text { C56 } \\
\text { (SUT0109056) }\end{array}$ & Bung Sam Pao, Muang District, Chainat & $\begin{array}{l}\mathrm{N} 15^{\circ} 16^{\prime} 5.9^{\prime \prime} \mathrm{E} 100^{\circ} 05^{\prime} \\
11.1^{\prime \prime} \text { Altitude } 41 \mathrm{~m}\end{array}$ & 482 & 39 & 8.09 \\
\hline $\begin{array}{l}\text { C57 } \\
\text { (SUT0109057) }\end{array}$ & Bird park Pond, Muang District, Chainat & $\begin{array}{l}\mathrm{N} 15^{\circ} 12^{\prime} 26.5^{\prime \prime} \mathrm{E} 100^{\circ} 09^{\prime} \\
21.9^{\prime \prime} \text { Altitude } 31 \mathrm{~m}\end{array}$ & 433 & 242 & 55.89 \\
\hline $\begin{array}{l}\text { C58 } \\
\text { (SUT0109058) }\end{array}$ & Fish Pond at Bird Park, Muang District, Chainat & $\begin{array}{l}\mathrm{N} 15^{\circ} 12^{\prime} 18.8^{\prime \prime} \mathrm{E} 100^{\circ} 09^{\prime} \\
\text { 20.0" Altitude } 39 \mathrm{~m}\end{array}$ & 885 & 41 & 4.63 \\
\hline $\begin{array}{l}\text { C59 } \\
\text { (SUT0109059) }\end{array}$ & $\begin{array}{l}\text { Khun Daan Prakarnchon Dam, Muang District, } \\
\text { Nakhonnayok }\end{array}$ & $\begin{array}{l}\text { N } 14^{\circ} 18^{\prime} 36.5^{\prime \prime} \text { E } 101^{\circ} 19^{\prime} \\
14.3^{\prime \prime} \text { Altitude } 25 \mathrm{~m}\end{array}$ & 501 & 130 & 25.95 \\
\hline $\begin{array}{l}\text { C60 } \\
\text { (SUT0109060) }\end{array}$ & $\begin{array}{l}\text { Ban mai Phai Chedi, Kampangsaen District, } \\
\text { Nakhonpathom }\end{array}$ & $\begin{array}{l}\mathrm{N} 14^{\circ} 02^{\prime} 10.5^{\prime \prime} \mathrm{E} 100^{\circ} 03^{\prime} \\
27.3^{\prime \prime} \text { Altitude } 10 \mathrm{~m}\end{array}$ & 361 & 21 & 5.82 \\
\hline $\begin{array}{l}\text { C61 } \\
\text { (SUT0109061) }\end{array}$ & Rice paddy, Banglen District, Nakhonpathom & $\begin{array}{l}\mathrm{N} 14^{\circ} 01^{\prime} 57.1^{\prime \prime} \mathrm{E} 100^{\circ} 10^{\prime} \\
24.0^{\prime \prime} \text { Altitude } 5 \mathrm{~m}\end{array}$ & 260 & 38 & 14.62 \\
\hline $\begin{array}{l}\text { C62 } \\
\text { (SUT0109062) }\end{array}$ & $\begin{array}{l}\text { Rice Field at Nong Kra Done, Muang District, } \\
\text { Nakhonpathom }\end{array}$ & $\begin{array}{l}\mathrm{N} 13^{\circ} 52^{\prime} 41.6^{\prime \prime} \mathrm{E} 99^{\circ} 55^{\prime} \\
50.0^{\prime \prime} \text { Altitude } 14 \mathrm{~m}\end{array}$ & 276 & 22 & 7.97 \\
\hline $\begin{array}{l}\text { C63 } \\
\text { (SUT0109063) }\end{array}$ & $\begin{array}{l}\text { Pond at Silpakorn University, Muang District, } \\
\text { Nakhonpathom }\end{array}$ & $\begin{array}{l}\mathrm{N} 13^{\circ} 48^{\prime} 84.2^{\prime \prime} \mathrm{E} 100^{\circ} 02^{\prime} \\
64.5^{\prime \prime} \text { Altitude } 11 \mathrm{~m}\end{array}$ & 272 & 27 & 9.93 \\
\hline $\begin{array}{l}\text { C64 } \\
\text { (SUT0109064) }\end{array}$ & $\begin{array}{l}\text { Bung Bo Ra Ped Lake, Muang District, Nakhon } \\
\text { Sawan }\end{array}$ & $\begin{array}{l}\mathrm{N} 15^{\circ} 42^{\prime} 3.06^{\prime \prime} \mathrm{E} 100^{\circ} 10^{\prime} \\
28.1^{\prime \prime} \text { Altitude } 17 \mathrm{~m}\end{array}$ & 274 & 23 & 8.39 \\
\hline $\begin{array}{l}\text { C65 } \\
\text { (SUT0109065) }\end{array}$ & Klong bang Ta nai, Pak Kred District, Nontaburi & $\begin{array}{l}\mathrm{N} 13^{\circ} 57^{\prime} 10.1^{\prime \prime} \mathrm{E} 100^{\circ} 29^{\prime} \\
05.4^{\prime \prime} \text { Altitude } 15 \mathrm{~m}\end{array}$ & 380 & 41 & 10.79 \\
\hline $\begin{array}{l}\text { C66 } \\
\text { (SUT0109066) }\end{array}$ & Ratniyom Canal, Sai Noi District, Nontaburi & $\begin{array}{l}\mathrm{N} 14^{\circ} 04^{\prime} 17.8^{\prime \prime} \mathrm{E} 100^{\circ} 19^{\prime} \\
23.7^{\prime \prime} \text { Altitude } 9 \mathrm{~m}\end{array}$ & 568 & 139 & 24.47 \\
\hline $\begin{array}{l}\text { C67 } \\
\text { (SUT0109067) }\end{array}$ & $\begin{array}{l}\text { Pra Udom Canal, Lad Loom Kaew District, } \\
\text { Pathumtani }\end{array}$ & $\begin{array}{l}\mathrm{N} 14^{\circ} 01^{\prime} 31.0^{\prime \prime} \mathrm{E} 100^{\circ} 22^{\prime} \\
01.1^{\prime \prime} \text { Altitude } 14 \mathrm{~m}\end{array}$ & 49 & 12 & 24.49 \\
\hline $\begin{array}{l}\text { C68 } \\
\text { (SUT0109068) }\end{array}$ & $\begin{array}{l}\text { Na Mai Canal, Lad Loom Kaew District, Pathum- } \\
\text { tani }\end{array}$ & $\begin{array}{l}\text { N } 14^{\circ} 03^{\prime} 32.7^{\prime \prime} \text { E } 100^{\circ} 26^{\prime} \\
54.6^{\prime \prime} \text { Altitude } 22 \mathrm{~m}\end{array}$ & 95 & 9 & 9.47 \\
\hline $\begin{array}{l}\text { C69 } \\
\text { (SUT0109069) }\end{array}$ & Wat Ko Phai, Bang Ban District, Ayutthaya & $\begin{array}{l}\mathrm{N} 14^{\circ} 24^{\prime} 40.9^{\prime \prime} \mathrm{E} 100^{\circ} 26^{\prime} \\
44.6^{\prime \prime} \text { Altitude } 9 \mathrm{~m}\end{array}$ & 4 & 2 & 50 \\
\hline $\begin{array}{l}\text { C70 } \\
\text { (SUT0109070) }\end{array}$ & $\begin{array}{l}\text { Pond at Ban Ta Woong, Ta Woong District, } \\
\text { Lopburi }\end{array}$ & $\begin{array}{l}\text { N } 14^{\circ} 50^{\prime} 24.4^{\prime \prime} \mathrm{E} 100^{\circ} 28^{\prime} \\
21.4^{\prime \prime} \text { Altitude } 13 \mathrm{~m}\end{array}$ & 161 & 11 & 6.83 \\
\hline $\begin{array}{l}\text { C71 } \\
(\text { SUT0109071) }\end{array}$ & $\begin{array}{l}\text { Suan Ma Dua waterfall, Pattana Nikom District, } \\
\text { Lopburi }\end{array}$ & $\begin{array}{l}\text { N 14 55' 06.0" E } 101^{\circ} 13^{\prime} \\
09.2^{\prime \prime} \text { Altitude } 125 \text { m }\end{array}$ & 233 & 16 & 6.87 \\
\hline $\begin{array}{l}\text { C72 } \\
\text { (SUT0109072) }\end{array}$ & $\begin{array}{l}\text { Pasak Chonlasit Reservior, Pattana Nikom Dis- } \\
\text { trict, Lopburi }\end{array}$ & $\begin{array}{l}\mathrm{N} 14^{\circ} 56^{\prime} 22.2^{\prime \prime} \mathrm{E} 101^{\circ} 04^{\prime} \\
47.4^{\prime \prime} \text { Altitude } 44 \mathrm{~m}\end{array}$ & 15 & 1 & 6.67 \\
\hline $\begin{array}{l}\text { C73 } \\
\text { (SUT0109073) }\end{array}$ & Tam Ru Canal, Muang District, Samut Prakan & $\begin{array}{l}\mathrm{N} 13^{\circ} 30^{\prime} 54.7^{\prime \prime} \mathrm{E} 100^{\circ} 41^{\prime} \\
12.0^{\prime \prime} \text { Altitude } 1 \mathrm{~m}\end{array}$ & 99 & 0 & 0 \\
\hline $\begin{array}{l}\text { C74 } \\
\text { (SUT0109074) }\end{array}$ & $\begin{array}{l}\text { Prachachomchuen Canal, Ampawa District, } \\
\text { Samut Songkram }\end{array}$ & $\begin{array}{l}\mathrm{N} 13^{\circ} 25^{\prime} 06.7^{\prime \prime} \mathrm{E} 99^{\circ} 57^{\prime} \\
17.8^{\prime \prime} \text { Altitude } 4 \mathrm{~m} \\
\end{array}$ & 29 & 5 & 17.24 \\
\hline $\begin{array}{l}\text { C75 } \\
\text { (SUT0109075) }\end{array}$ & $\begin{array}{l}\text { Don Ko Canal, Ban Phaew District, Samut } \\
\text { Sakhon }\end{array}$ & $\begin{array}{l}\mathrm{N} 13^{\circ} 38^{\prime} 08.0^{\prime \prime} \mathrm{E} 100^{\circ} 05^{\prime} \\
\text { 03.0" Altitude } 7 \mathrm{~m}\end{array}$ & 42 & 6 & 14.29 \\
\hline $\begin{array}{l}\text { C76 } \\
\text { (SUT0109076) }\end{array}$ & Ta Pa Canal, Ban Phaew District, Samut Sakhon & $\begin{array}{l}\mathrm{N} 13^{\circ} 38^{\prime} 07.3^{\prime \prime} \mathrm{E} 100^{\circ} 06^{\prime} \\
20.1^{\prime \prime} \text { Altitude } 30 \mathrm{~m}\end{array}$ & 85 & 1 & 1.18 \\
\hline $\begin{array}{l}\text { C77 } \\
\text { (SUT0109077) }\end{array}$ & Muak Lek Waterfall, Muak Lek District, Saraburi & $\begin{array}{l}\mathrm{N} 14^{\circ} 43^{\prime} 13.2^{\prime \prime} \mathrm{E} 101^{\circ} 11^{\prime} \\
19.4^{\prime \prime} \text { Altitude } 156 \mathrm{~m}\end{array}$ & 199 & 2 & 1.01 \\
\hline $\begin{array}{l}\text { C78 } \\
\text { (SUT0109078) }\end{array}$ & $\begin{array}{l}\text { Dong Phya Yen Waterfall, Muak Lek District, } \\
\text { Saraburi }\end{array}$ & $\begin{array}{l}\text { N } 14^{\circ} 44^{\prime} 0.6^{\prime \prime} \mathrm{E} 101^{\circ} 11^{\prime} \\
44.6^{\prime \prime} \text { Altitude } 162 \mathrm{~m}\end{array}$ & 180 & 5 & 2.78 \\
\hline $\begin{array}{l}\text { C79 } \\
\text { (SUT0109079) }\end{array}$ & $\begin{array}{l}\text { Site1 at Ched Kot Waterfall, Kaeng Koi District, } \\
\text { Saraburi }\end{array}$ & $\begin{array}{l}\mathrm{N} 14^{\circ} 28^{\prime} 48.5^{\prime \prime} \mathrm{E} 101^{\circ} 10^{\prime} \\
22.3^{\prime \prime} \text { Altitude } 185 \mathrm{~m}\end{array}$ & 118 & 39 & 33.05 \\
\hline $\begin{array}{l}\text { C80 } \\
\text { (SUT0109080) }\end{array}$ & $\begin{array}{l}\text { Site2 at Ched Kot waterfall, Kaeng Koi District, } \\
\text { Saraburi }\end{array}$ & $\begin{array}{l}\mathrm{N} 14^{\circ} 28^{\prime} 34.6^{\prime \prime} \mathrm{E} 101^{\circ} 10^{\prime} \\
16.4^{\prime \prime} \text { Altitude } 157 \mathrm{~m}\end{array}$ & 37 & 12 & 32.43 \\
\hline $\begin{array}{l}\text { C81 } \\
\text { (SUT0109081) }\end{array}$ & $\begin{array}{l}\text { Reservoir at Sam Lan Waterfall, Muang District, } \\
\text { Saraburi }\end{array}$ & $\begin{array}{l}\text { N } 14^{\circ} 25^{\prime} 52.2^{\prime \prime} \text { E } 100^{\circ} 57^{\prime} \\
49.6^{\prime \prime} \text { Altitude } 88 \mathrm{~m}\end{array}$ & 315 & 8 & 2.54 \\
\hline $\begin{array}{l}\text { C82 } \\
\text { (SUT0109082) }\end{array}$ & Muang Moo pond, Muang District, Singhaburi & $\begin{array}{l}\mathrm{N} 14^{\circ} 52^{\prime} 09.1^{\prime \prime} \mathrm{E} 100^{\circ} 24^{\prime} \\
59.1^{\prime \prime} \text { Altitude } 16 \mathrm{~m}\end{array}$ & 282 & 29 & 10.28 \\
\hline
\end{tabular}




\begin{tabular}{|c|c|c|c|c|c|}
\hline $\begin{array}{l}\text { No. } \\
\text { (no. specimen) }\end{array}$ & Name & GPS & \begin{tabular}{|c|}
$\begin{array}{c}\text { No. of } \\
\text { collected } \\
\text { snails }\end{array}$ \\
\end{tabular} & \begin{tabular}{|c|} 
No. of \\
Infected \\
snails
\end{tabular} & $\begin{array}{c}\text { Infection } \\
\text { rates } \\
(\%) \\
\end{array}$ \\
\hline $\begin{array}{l}\text { C83 } \\
\text { (SUT0109083) }\end{array}$ & $\begin{array}{l}\text { Ban Bang Mae Mai Pond, Bang Pla Ma District, } \\
\text { Suphanburi }\end{array}$ & $\begin{array}{l}\mathrm{N} 14^{\circ} 20^{\prime} 32.2^{\prime \prime} \mathrm{E} 100^{\circ} 09^{\prime} \\
04.9^{\prime \prime} \text { Altitude } 8 \text { m }\end{array}$ & 349 & 85 & 24.36 \\
\hline $\begin{array}{l}\text { C84 } \\
\text { (SUT0109084) }\end{array}$ & $\begin{array}{l}\text { Wat bang Yai Pond, Bang Pla Ma District, } \\
\text { Suphanburi }\end{array}$ & $\begin{array}{l}\mathrm{N} 14^{\circ} 18^{\prime} 41.2^{\prime \prime} \mathrm{E} 100^{\circ} 09^{\prime} \\
03.7^{\prime \prime} \text { Altitude } 5 \mathrm{~m}\end{array}$ & 956 & 46 & 4.81 \\
\hline $\begin{array}{l}\text { C85 } \\
\text { (SUT0109085) }\end{array}$ & $\begin{array}{l}\text { Bung Cha Wak Pond, Derm Bang Nang Buad } \\
\text { District, Supahanburi }\end{array}$ & $\begin{array}{l}\mathrm{N} 14^{\circ} 54^{\prime} 04.4^{\prime \prime} \mathrm{E} 100^{\circ} 03^{\prime} \\
\text { 48.0" Altitude } 26 \mathrm{~m}\end{array}$ & 176 & 30 & 17.05 \\
\hline $\begin{array}{l}\text { C86 } \\
\text { (SUT0109086) }\end{array}$ & Huay Po Canal, Muang District, Angthong & $\begin{array}{l}\mathrm{N} 14^{\circ} 36^{\prime} 08.3^{\prime \prime} \mathrm{E} 100^{\circ} 24^{\prime} \\
12.9^{\prime \prime} \text { Altitude } 14 \mathrm{~m} \\
\end{array}$ & 65 & 10 & 15.38 \\
\hline \multicolumn{6}{|l|}{ South } \\
\hline $\begin{array}{l}\text { S87 } \\
\text { (SUT0109087) }\end{array}$ & $\begin{array}{l}\text { Water Gate km. 19+500, Cha-Am District, } \\
\text { Petchaburi }\end{array}$ & $\begin{array}{l}\mathrm{N} 12^{\circ} 51^{\prime} 15.1^{\prime \prime} \mathrm{E} 99^{\circ} 59^{\prime} \\
48.5^{\prime \prime} \text { Altitude } 17 \mathrm{~m}\end{array}$ & 95 & 4 & 4.21 \\
\hline $\begin{array}{l}\text { S88 } \\
\text { (SUT0109088) }\end{array}$ & $\begin{array}{l}\text { Emergency Water Gate, Cha-Am District, Petch- } \\
\text { aburi }\end{array}$ & $\begin{array}{l}\mathrm{N} 12^{\circ} 57.4^{\prime} 26.0^{\prime \prime} \mathrm{E} 100^{\circ} 02^{\prime} \\
07.5^{\prime \prime} \text { Altitude } 14 \mathrm{~m}\end{array}$ & 3 & 0 & 0 \\
\hline $\begin{array}{l}\text { S89 } \\
\text { (SUT0109089) }\end{array}$ & Petchaburi Dam, Tayang District, Petchaburi & $\begin{array}{l}\text { N } 12^{\circ} 54^{\prime} 58.6^{\prime \prime} \text { E } 99^{\circ} 51^{\prime} \\
34.4^{\prime \prime} \text { Altitude } 20 \mathrm{~m}\end{array}$ & 137 & 5 & 3.65 \\
\hline $\begin{array}{l}\text { S90 } \\
\text { (SUT0109090) }\end{array}$ & $\begin{array}{l}\text { Pond at Silpakorn University Petchaburi Cam- } \\
\text { pus, Cha-Am District, Petchaburi }\end{array}$ & $\begin{array}{l}\mathrm{N} 11^{\circ} 26^{\prime} 04.6^{\prime \prime} \text { E } 99^{\circ} 26^{\prime} \\
56.9^{\prime \prime} \text { Altitude } 90 \mathrm{~m}\end{array}$ & 1,074 & 1,051 & 97.86 \\
\hline $\begin{array}{l}\text { S91 } \\
\text { (SUT0109091) }\end{array}$ & $\begin{array}{l}\text { Huai Yang stream, Huai Yang District, Prachuab- } \\
\text { kirikhun }\end{array}$ & $\begin{array}{l}\mathrm{N} 11^{\circ} 36^{\prime} 47.0^{\prime \prime} \mathrm{E} 99^{\circ} 40^{\prime} \\
08.4^{\prime \prime} \text { Altitude } 18 \mathrm{~m}\end{array}$ & 211 & 30 & 14.22 \\
\hline $\begin{array}{l}\text { S92 } \\
\text { (SUT0109092) }\end{array}$ & $\begin{array}{l}\text { Thap sakae stream, Tuap sakae District, Prach- } \\
\text { uabkirikhun }\end{array}$ & $\begin{array}{l}\mathrm{N} 11^{\circ} 29^{\prime} 40.1^{\prime \prime} \mathrm{E} 99^{\circ} 36^{\prime} \\
20.3^{\prime \prime} \text { Altitude } 13 \mathrm{~m}\end{array}$ & 124 & 8 & 6.45 \\
\hline $\begin{array}{l}\text { S93 } \\
\text { (SUT0109093) }\end{array}$ & $\begin{array}{l}\text { Kha On waterfall, Bangsapan District, Prachuab- } \\
\text { kirikhun }\end{array}$ & $\begin{array}{l}\mathrm{N} 11^{\circ} 26^{\prime} 04.6^{\prime \prime} \text { E } 99^{\circ} 26^{\prime} \\
56.9^{\prime \prime} \text { Altitude } 90 \mathrm{~m}\end{array}$ & 32 & 4 & 12.50 \\
\hline $\begin{array}{l}\text { S94 } \\
\text { (SUT0109094) }\end{array}$ & $\begin{array}{l}\text { Sai Khu waterfall, Bangsapan District, Prachuab- } \\
\text { kirikhun }\end{array}$ & $\begin{array}{l}\mathrm{N} 11^{\circ} 14^{\prime} 21.8^{\prime \prime} \mathrm{E} 99^{\circ} 21^{\prime} \\
36.1^{\prime \prime} \text { Altitude } 83 \mathrm{~m}\end{array}$ & 429 & 25 & 5.83 \\
\hline $\begin{array}{l}\text { S95 } \\
\text { (SUT0109095) }\end{array}$ & Kapoh Waterfall, Ta Sae District, Chumporn & $\begin{array}{l}\text { N } 10^{\circ} 44^{\prime} 28.7^{\prime \prime} \text { E } 99^{\circ} 12^{\prime} \\
53.9^{\prime \prime} \text { Altitude } 69 \mathrm{~m}\end{array}$ & 133 & 5 & 3.76 \\
\hline $\begin{array}{l}\text { S96 } \\
\text { (SUT0109096) }\end{array}$ & Ra Canal, Lungsuan District, Chumporn & 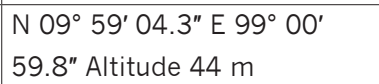 & 739 & 97 & 13.13 \\
\hline $\begin{array}{l}\text { S97 } \\
\text { (SUT0109097) }\end{array}$ & $\begin{array}{l}\text { Si Kheed Waterfall, Sichon District, Nakhon Sri } \\
\text { Thammarat }\end{array}$ & $\begin{array}{l}\mathrm{N} 09^{\circ} 00^{\prime} 40.8^{\prime \prime} \mathrm{E} 99^{\circ} 46^{\prime} \\
30.1^{\prime \prime} \text { Altitude } 45 \mathrm{~m}\end{array}$ & 9 & 1 & 11.11 \\
\hline $\begin{array}{l}\text { S98 } \\
\text { (SUT0109098) }\end{array}$ & $\begin{array}{l}\text { Krung Ching Waterfall, Noppitum District, Nak- } \\
\text { hon Sri Thammarat }\end{array}$ & $\begin{array}{l}\mathrm{N} 08^{\circ} 43^{\prime} 14.0^{\prime \prime} \mathrm{E} 99^{\circ} 40^{\prime} \\
15.2^{\prime \prime} \text { Altitude } 45 \mathrm{~m}\end{array}$ & 18 & 5 & 27.78 \\
\hline $\begin{array}{l}\text { S99 } \\
\text { (SUT0109099) }\end{array}$ & $\begin{array}{l}\text { Yod Leung Stream, Noppitum District, Nakhon } \\
\text { Sri Thammarat }\end{array}$ & $\begin{array}{l}\mathrm{N} 08^{\circ} 38^{\prime} 10.5^{\prime \prime} \mathrm{E} 99^{\circ} 45^{\prime} \\
11.6^{\prime \prime} \text { Altitude } 68 \mathrm{~m}\end{array}$ & 872 & 343 & 0.39 \\
\hline $\begin{array}{l}\text { S100 } \\
\text { (SUT0109100) }\end{array}$ & Palian River, Yan Ta Khao District, Trang & $\begin{array}{l}\text { N } 07^{\circ} 22^{\prime} 11.5^{\prime \prime} \text { E } 99^{\circ} 40^{\prime} \\
51.6^{\prime \prime} \text { Altitude } 12 \mathrm{~m}\end{array}$ & 205 & 22 & 10.73 \\
\hline $\begin{array}{l}\text { S101 } \\
\text { (SUT0109101) }\end{array}$ & Palian Dam, Palien District, Trang & $\begin{array}{l}\mathrm{N} 07^{\circ} 19^{\prime} 13.2^{\prime \prime} \mathrm{E} 99^{\circ} 48^{\prime} \\
28.8^{\prime \prime} \text { Altitude } 43 \mathrm{~m}\end{array}$ & 506 & 46 & 9.09 \\
\hline $\begin{array}{l}\text { S102 } \\
\text { (SUT0109102) }\end{array}$ & Tone Tok Waterfall, Palien District, Trang & $\begin{array}{l}\mathrm{N} 07^{\circ} 16^{\prime} 44.2^{\prime \prime} \text { E } 99^{\circ} 53^{\prime} \\
10.6^{\prime \prime} \text { Altitude } 41 \mathrm{~m}\end{array}$ & 301 & 78 & 25.91 \\
\hline $\begin{array}{l}\text { S103 } \\
\text { (SUT0109103) }\end{array}$ & Tone Plew Waterfall, Nayong District, Trang & $\begin{array}{l}\mathrm{N} 07^{\circ} 32^{\prime} 48.2^{\prime \prime} \mathrm{E} 99^{\circ} 47^{\prime} \\
17.0^{\prime \prime} \text { Altitude } 63 \mathrm{~m}\end{array}$ & 1,049 & 97 & 0.09 \\
\hline $\begin{array}{l}\text { S104 } \\
\text { (SUT0109104) }\end{array}$ & Ang Thong Waterfall, Sikao District, Trang & 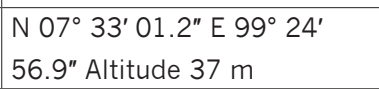 & 311 & 107 & 34.41 \\
\hline $\begin{array}{l}\text { S105 } \\
\text { (SUT0109105) }\end{array}$ & Falan Waterfall, Srinakarin District, Pattaloong & $\begin{array}{l}\mathrm{N} 07^{\circ} 36^{\prime} 25.9^{\prime \prime} \mathrm{E} 99^{\circ} 54^{\prime} \\
39.0^{\prime \prime} \text { Altitude } 80 \mathrm{~m}\end{array}$ & 1 & 0 & 0 \\
\hline $\begin{array}{l}\text { S106 } \\
\text { (SUT0109106) }\end{array}$ & Ban Au Rua Stream, Muang District, Ratchaburi & $\begin{array}{l}\mathrm{N} 13^{\circ} 31^{\prime} 21.9^{\prime \prime} \mathrm{E} 99^{\circ} 50^{\prime} \\
57.3^{\prime \prime} \text { Altitude } 152 \mathrm{~m}\end{array}$ & 9 & 2 & 22.22 \\
\hline $\begin{array}{l}\text { S107 } \\
\text { (SUT0109107) }\end{array}$ & $\begin{array}{l}\text { Huay Haeng Stream, Suanphueng District, } \\
\text { Ratchaburi }\end{array}$ & $\begin{array}{l}\mathrm{N} 13^{\circ} 31^{\prime} 03.9^{\prime \prime} \text { E } 99^{\circ} 20^{\prime} \\
29.2^{\prime \prime} \text { Altitude } 113 \mathrm{~m}\end{array}$ & 213 & 18 & 8.45 \\
\hline $\begin{array}{l}\text { S108 } \\
\text { (SUT0109108) }\end{array}$ & $\begin{array}{l}\text { Huay Nuang Stream, Suanphueng District, } \\
\text { Ratchaburi }\end{array}$ & $\begin{array}{l}\mathrm{N} 13^{\circ} 31^{\prime} 21.9^{\prime \prime} \mathrm{E} 99^{\circ} 17^{\prime} \\
36.5^{\prime \prime} \text { Altitude } 151 \mathrm{~m}\end{array}$ & 234 & 23 & 9.83 \\
\hline $\begin{array}{l}\text { S109 } \\
\text { (SUT0109109) }\end{array}$ & Bangborn Stream, Kraburi District, Ranong & $\begin{array}{l}\mathrm{N} 10^{\circ} 20^{\prime} 10.8^{\prime \prime} \text { E } 98^{\circ} 46^{\prime} \\
48.7^{\prime \prime} \text { Altitude } 18 \mathrm{~m}\end{array}$ & 34 & 2 & 5.88 \\
\hline
\end{tabular}




\begin{tabular}{|c|c|c|c|c|c|}
\hline $\begin{array}{c}\text { No. } \\
\text { (no. specimen) }\end{array}$ & Name & GPS & $\begin{array}{c}\text { No. of } \\
\text { collected } \\
\text { snails }\end{array}$ & $\begin{array}{l}\text { No. of } \\
\text { Infected } \\
\text { snails }\end{array}$ & $\begin{array}{c}\text { Infection } \\
\text { rates } \\
(\%)\end{array}$ \\
\hline $\begin{array}{l}\text { S110 } \\
\text { (SUT0109110) }\end{array}$ & $\begin{array}{l}\text { Na Ca Stream, Wild Life Sancuatuary, Muang } \\
\text { District, Ranong }\end{array}$ & $\begin{array}{l}\mathrm{N}^{\circ} 27^{\prime} 26.6^{\prime \prime} \mathrm{E} 98^{\circ} 30^{\prime} 36.9^{\prime \prime} \\
\text { Altitude } 3 \mathrm{~m}\end{array}$ & 56 & 1 & 1.79 \\
\hline $\begin{array}{l}\text { S111 } \\
\text { (SUT0109111) }\end{array}$ & $\begin{array}{l}\text { Wiphawadee Waterfall, Donsak District, Surat } \\
\text { Thani }\end{array}$ & $\begin{array}{l}\mathrm{N} 9^{\circ} 8^{\prime} 9.6^{\prime \prime} \mathrm{E} 99^{\circ} 40^{\prime} 31.2^{\prime \prime} \\
\text { Altitude } 10 \mathrm{~m}\end{array}$ & 70 & 2 & 2.86 \\
\hline $\begin{array}{l}\text { S112 } \\
\text { (SUT0109112) }\end{array}$ & Yan Canal, Wipawadee District, Surathani & $\begin{array}{l}\text { N 9०12'12.8" E 9857'20.3" } \\
\text { Altitude } 66 \text { m }\end{array}$ & 292 & 21 & 7.19 \\
\hline $\begin{array}{l}\text { S113 } \\
\text { (SUT0109113) }\end{array}$ & Ton Sai Waterfall, Tha Lang District, Phuket & $\begin{array}{l}\text { N } 8^{\circ} 1^{\prime} 32.4^{\prime \prime} \text { E } 98^{\circ} 21^{\prime} 58.8^{\prime \prime} \\
\text { Altitude } 45 \text { m }\end{array}$ & 222 & 27 & 12.16 \\
\hline $\begin{array}{l}\text { S114 } \\
\text { (SUT0109114) }\end{array}$ & Bang Pae waterfall, Tha Lang District, Phuket & $\begin{array}{l}\text { N 8 2' } 20.5^{\prime \prime} \text { E } 98^{\circ} 23^{\prime} 49.3^{\prime \prime} \\
\text { Altitude } 50 \mathrm{~m}\end{array}$ & 76 & 13 & 17.11 \\
\hline $\begin{array}{l}\text { S115 } \\
\text { (SUT0109115) }\end{array}$ & Kathu Waterfall, Kathu District, Phuket & $\begin{array}{l}\text { N 755' 49.4" E 98 19'34" } \\
\text { Altitude } 43 \text { m }\end{array}$ & 385 & 5 & 1.30 \\
\hline $\begin{array}{l}\text { S116 } \\
\text { (SUT0109116) }\end{array}$ & $\begin{array}{l}\text { Raman Waterfall, Ta Kua Tung District, Phang } \\
\text { Nga }\end{array}$ & $\begin{array}{l}\text { N 8 27' } 8.5^{\prime \prime} \text { E } 98^{\circ} 28^{\prime} 0.9^{\prime \prime} \\
\text { Altitude } 33 \text { m }\end{array}$ & 3 & 0 & 0 \\
\hline $\begin{array}{l}\text { S117 } \\
\text { (SUT0309117) }\end{array}$ & Sa Morakot Stream, Klong Tom District, Krabi & $\begin{array}{l}\mathrm{N} 7^{\circ} 55^{\prime} 14.9^{\prime \prime} \text { E } 99^{\circ} 15^{\prime} 47.1^{\prime \prime} \\
\text { Altitude } 24 \mathrm{~m}\end{array}$ & 356 & 4 & 1.12 \\
\hline $\begin{array}{l}\text { S118 } \\
\text { (SUT0109118) }\end{array}$ & Panan Waterfall, Kuan galung District, Satoon & $\begin{array}{l}\mathrm{N} \mathrm{6}^{\circ} 51^{\prime} 22.8^{\prime \prime} \text { E } 100^{\circ} 9^{\prime} 48.6^{\prime \prime} \\
\text { Altitude } 47 \mathrm{~m}\end{array}$ & 170 & 6 & 3.53 \\
\hline $\begin{array}{l}\text { S119 } \\
\text { (SUT0109119) }\end{array}$ & Tha Phae Dam, Kuan Done District, Satoon & $\begin{array}{l}\mathrm{N} 6^{\circ} 49^{\prime} 26^{\prime \prime} \mathrm{E} 100^{\circ} 2^{\prime 2} 2.3^{\prime \prime} \\
\text { Altitude } 41 \mathrm{~m}\end{array}$ & 760 & 8 & 1.05 \\
\hline $\begin{array}{l}\text { S120 } \\
\text { (SUT0109120) }\end{array}$ & Klong Muang, Kuan Niang District, Songkhla & $\begin{array}{l}\mathrm{N} 7^{\circ} 12^{\prime} 24.5^{\prime \prime} \mathrm{E} 100^{\circ} 22^{\prime} 43.1^{\prime \prime} \\
\text { Altitude } 13 \mathrm{~m}\end{array}$ & 82 & 9 & 10.98 \\
\hline
\end{tabular}

(N 19²5'31.7’', E 9759'24.9”, Altitude 343 m); N6 = Huay Sua Thao Stream, Muang District, Mae Hong Son Province (SUT0109006) (N 19¹5’32.0”, E 9754'43.7', Altitude $237 \mathrm{~m}$ ); N7 = Klong Nam Lai Waterfall, Klong Lan District, Kam Phaeng Phet Province (SUT0109007) (N 16¹1'32.7'”, E 99¹5'51.0”, Altitude $241 \mathrm{~m}$ ); N8 = Tad Duen Waterfall, Sri Satchanalai District, Sukhothai Province (SUT0109008) (N 17³3'23.2”'E 99²9'76.8”, Altitude 414 m); N9 = Sri Satchanalai Stream, Sri Satchanalai National Park, Sukhothai Province (SUT0109009) (N 17³3'5.9', E 99²9'24.8', Altitude $182 \mathrm{~m}$ ); N10 = Mae Pool Waterfall, Lub Lae District, Ut-taradit Province (SUT0109010) (N 1743’45.0”, E 9958'50.6”, Altitude $164 \mathrm{~m}) ; \mathrm{N} 11$ = Chueng Thong Waterfall, Muang District, Prae Province (SUT0109011) (N 1801'54.2”, E $100^{\circ} 15^{\prime} 52.8^{\prime \prime}$, Altitude $298 \mathrm{~m}$ ); N12 = Huay Sa Nien Stream, Muang District, Nan Province (SUT0109012) (N 18 51'1.3”, E 100³9'16.2”, Altitude 280 m); N13 = Huay Ton Phueng Waterfall, Doi Pu Nang National Park, Chiang Muon District, Phyao Province (SUT0109013) (N 1855'5.3”, E 100¹2'15.7’', Altitude 379); N14 = Tansawan Waterfall, Doi Pu Nang National Park, Chiang Muon District, Phyao Province (SUT0109014) (N 18 51'22.7', E 100¹1'9.6”, Altitude 420 m); N15 = Mae Mine Stream, Mae Ta District, Lampang Province (SUT0109015) (N 1807'1.8”, E 99³7’35.1”, Altitude $269 \mathrm{~m})$.

The Northeast: NE $16=$ Huay Lum Po Dang Stream, Thepsathit District, Chaiyapoom Province (SUT0109016) (N 15³3'42.8”, E 101²4'56.9”, Altitude $471 \mathrm{~m})$; NE 17 = Sai Thong Waterfall, Sai Thong Nation- al Park, Nong Bua Ra Hoew District, Chaiyapoom Province (SUT0109017) (N 1552’34.7’, E 101³0’34.7’, Altitude $397 \mathrm{~m}$ ); NE18 = Tad Tone Waterfall, Muang District, Chaiyappom Porvince (SUT0109018) (N 15'58'42.5”, E 10202'24.9”, Altitude 384 m); NE19 = Kongkaew Waterfall, Khao Yai National Park, Pak Chong District, Nakhon Ratchasima Province (SUT0109019) (N 14²6'14.8', E 101²2'37.6”, Altitude $713 \mathrm{~m}$ ); NE20 = Lam Takhong Stream, Khao Yai National Park, Pak Chong District, Nakhon Ratchasima Province (SUT0109020) (N 14²5'19.6”, E 101²3'26.3”, Altitude $700 \mathrm{~m})$; NE21 = Ban Cha Rut Reservoir, Bua Ched District, Surin Province (SUT0109021) (N 14²5'50.4”, E 103'57'47.7”, Altitude $201 \mathrm{~m}$ ); NE22 = Nong Bua Rai Reservoir, Khao Panomroong, Pra Kone Chai District, Burirum Province (SUT0109022) (N 14³2'51.2”, E 102॰58'9.4", Altitude 202 m); NE23 = Pla Ba Waterfall, Pu Rua District, Loei Province (SUT0109023) (N 17²3'24.3”, E 101²2'27.4”, Altitude 640 m); NE24 = Than Thong Waterfall, Sung Kom District, Nong Khai Province (SUT0109024) (N 1801'34.7”, E 102²2'8.7”, Altitude $195 \mathrm{~m})$; NE25 = Huay Hor Water Gate, Muang District, Nakhon Panom Province (SUT0109025) (N 17²1'8.4”, E 10447'2.1'”, Altitude $145 \mathrm{~m}$ ); NE26 = Tad Kham Waterfall, Pu Lung Ga National Park, Ban Pang District, Nakhon Panom province (SUT0109026) (N 1757'1.4”, E 10409'39.9”, Altitude 148 m); NE27 = Tad Po Waterfall, Pu Lung Ga National Park, Ban Pang District, Nakhon Panom Province (SUT0109027) (N 1759'0.9', E 10408'34.3”, Altitude 148 m); NE28 = Nong Han, Muang Distrcit, Sakol Nakhon Prov- 
ince (SUT0109028) (N 1709'50.1”, E 10409'43.7”, Altitude $161 \mathrm{~m}$ ); NE29 = Nam Poong Dam, Gud Bak Distrcit, Sakol Nakhon Province (SUT0109029) (N 1658'11.8”, E 10359'13.4”, Altitude 290 m); NE30 = Lam Pow Dam, Muang District, Sri Sa Ket Province (SUT0109030) (N 16³6'22.3”, E 103²6'27.5”, Altitude $165 \mathrm{~m}$ ); NE31 = Ban Nong Wang Wong Reservior, Sri Som Det District, Roi-Et Province (SUT0109031) (N 1556'53.5”, E 10331'27.8”, Altitude $178 \mathrm{~m}$ ); NE32 = Bung Toong Sang, Muang District, Khon Kaen Province (SUT0109032) (N 16²6'27.8”, E 102 51'28.1”, Altitude 154 m); NE33 = Bung Kaen Nakhon, Muang District, Khon Kaen Province (SUT0109033) (N 16²4'46.6”, E 102'50'21.9”, Altitude 143 m); NE34 = Nong Sa Ard Bamroong Reservoir, Kosum Pisai District, Mahasarkham Province (SUT0109034) (N 16 18'0.5”, E 102 54'38.5", Altitude $169 \mathrm{~m}$ ); NE35 = Tad Tone Waterfall, Nong Soong District, Mukdaharn Province (SUT0109035) (N 16²9’34.9”, E 104¹9'1.1”, Altitude $219 \mathrm{~m}$ )

The East: E36 = Khao Khaew National Park, Sriracha District, Chonburi Province (SUT0109036) (N $13^{\circ} 12^{\prime} 45.0^{\prime \prime}, \mathrm{E} 101^{\circ} 03^{\prime} 50.2^{\prime \prime}$, Altitude $128 \mathrm{~m}$ ); E37 $=$ Ban Nong Pla Lai, Bang lamung District, Chonburi Province (SUT0109037) (N 1257'54.3”, E $100^{\circ} 56^{\prime} 47.8^{\prime \prime}$, Altitude $17 \mathrm{~m}$ ); E38 = Rayong River, Muang District, Rayong Province (SUT0109038) (N 12³9'52.6”, E 101¹4'48.5”, Altitude 6 m); E39 $=$ Hin Khao Canal, Muang District, Rayong Province (SUT0109039) (N 12³6'31.7’, E 101²3'22.4”, Altitude $1 \mathrm{~m}$ ); E40 = Pung rad Canal, Klang District, Rayong Province (SUT0109040) (N 1242'49.5”, E 101'46'23.4", Altitude $15 \mathrm{~m}$ ); E41 = Chantaburi River, Muang District, Chantaburi Province (SUT0109041) (N 12³6'13.8”, E 10207'11.6”, Altitude 8 m); E42 = Nam Tok Plew Stream, Plew District, Chantaburi Province (SUT0109042) (N 12³1'14.3”, E 102 10'35.4”, Altitude $39 \mathrm{~m}$ ); E43 = Pa Tong Canal, Soi Dao District, Chantaburi Province (SUT0109043) (N 1307'5.9”, E $102^{\circ} 13^{\prime} 13.6$ ', Altitude $231 \mathrm{~m}$ ); E44 = Klong Kaew Waterfall, Bo Rai District, Trad Province (SUT0109044) (N 12³7’3.0”, E 102`34'52.0”, Altitude $81 \mathrm{~m})$; E45 = Sra Kaew, Muang District, Srakaew Province (SUT0109045) (N 1349'7.0", E 10203'37.9”, Altitude 43 m); E46 = Eto Waterfall, Muang District, Prachinburi Province (SUT0109046) (N 1408'58.9", E 101²4'45.4", Altitude $39 \mathrm{~m}$ ).

The Central: $\mathrm{C} 47=$ Dusit Zoo Pond, Dusit, Bangkok (SUT0109047) (N 1346’17.4”, E 100³1'14.8”, Altitude $2 \mathrm{~m}$ ); $\mathrm{C} 48$ = Drainage at Kasetsart University, Bang Khen Campus, Bangkok (SUT0109048) (N 1350'40.7”, E 100³4'33.5”, Altitude $5 \mathrm{~m}$ ); C49 = Pond at Kasetsart University, Bang Khen Campus, Bangkok (SUT0109049) (N 1350’22.6”, E 100³4'43.4”, Altitude $1 \mathrm{~m}$ ); C50 = Hin Dad Waterfall, Thong Pa Poom District, Kanchanaburi Province (SUT0109050) (N 14³7'29.8”, E 9843'40.2”, 186 m); C51 = Pha Tad Waterfall, Sri Sa wat District, Kanchanaburi Province

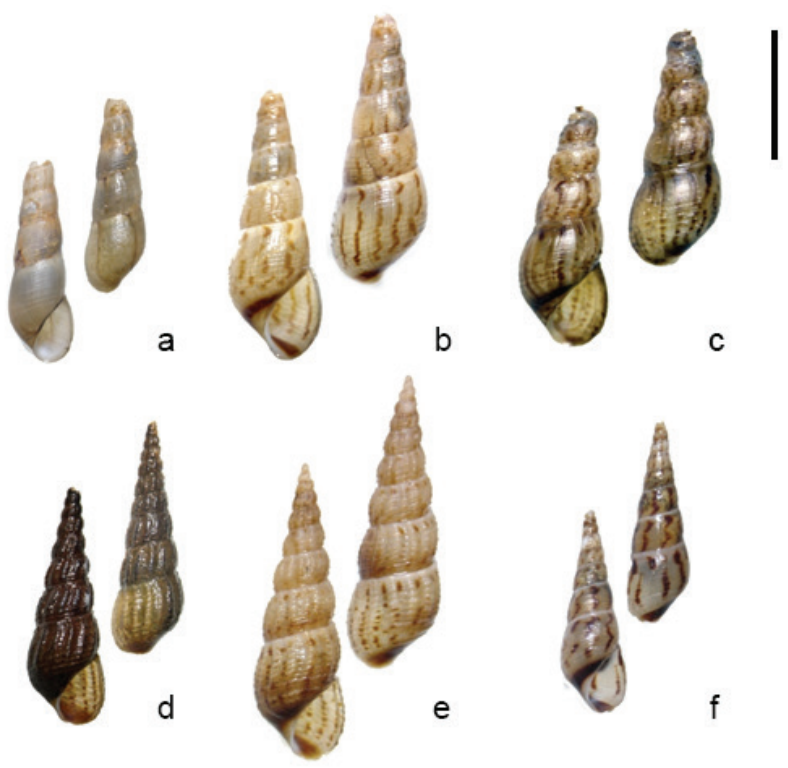

Figure 2. Shell Morphology of Melanoides tuberculata in Thailand a. Sri Satchanalai Stream, Sri Satchanalai National Park, Sri Satchanalai District, Sukhothai Province (SUT0109009)

b. Lam Takhong Stream, Khao Yai National Park, Pak Chong District, Nakhon, Ratchasima Province (SUT0109020)

c. Rice Field at Nong Kra Done, Muang District, Nakhon Pathom Province (SUT0109062)

d. Pond in Silpakorn University, Muang District, Nakhon Pathom Province (SUT0109063)

e. Bang Ta-nai Canal, Pak Kred District, Nontaburi Province (SUT0109065)

f. Huay Nuang Stream, Suanphueng District, Ratchaburi Province (SUT0109108)

Scale bar $=1.0 \mathrm{~mm}$.

(SUT0109051) (N 14³8'54.9”, E 9846’41.6”, Altitude $196 \mathrm{~m}$ ); C52 = Sai Yok Noi Waterfall, Sai Yok District, Kanchanaburi Province (SUT0109051) (N 14¹4'27.6”, E 9903'55.9”, Altitude 166 m); C53 = Sai Yok Yai Waterfall, Sai Yok District, Kanchanaburi Province (SUT0109053) (N 14²6’03.0”, E 98 51'14.7’, Altitude $140 \mathrm{~m}$ ); C54 = Wans Soong Canal, Bang Kla District, Chachuengsao Province (SUT0109054) (N 13³9'46.2”, E 101¹0'48.2”, Altitude 18 m); C55 = Sua Noi Canal, Bnag pa Kong District, Chachuengsao Province (SUT0109055) (N 133'31.0”, E 10057'13.8”, Altitude $2 \mathrm{~m}$ ); C56 = Bung Sam Pao, Muang District, Chainat Province (SUT0109056) (N 15¹6'5.9”, E $100^{\circ} 05^{\prime} 11.1$ ', Altitude $41 \mathrm{~m}$ ); C57 = Bird Park Pond, Muang District, Chainat Province (SUT0109057) (N $15^{\circ} 12^{\prime} 26.5^{\prime \prime}, \mathrm{E} 100^{\circ} 09^{\prime} 21.9^{\prime \prime}$, Altitude $\left.31 \mathrm{~m}\right)$; C58 = Fish Pond at Bird Park, Muang District, Chainat Province (SUT0109058) (N 15²12'18.8”, E 10009'20.0”, Altitude 39 m); C59 = Khun Daan Prakarnchon Dam, Muang District, Nakhonnayok Province (SUT0109059) (N 1418'36.5”, E 101¹9'14.3”, Altitude 25m); C60 = Ban mai Phai Chedi, Kampangsaen District, Nakhonpathom Province (SUT0109060) (N 1402'10.5”, E $100^{\circ} 03$ '27.3”, Altitude $10 \mathrm{~m}$ ); C61 = Rice paddy, Ban- 
glen District, Nakhonpathom Province (SUT0109061) (N 1401'57.1”, E 100¹0'24.0”, Altitude 5m); C62 $=$ Rice Field at Nong Kra Done, Muang District, nakhon Pathom Province (SUT0109062) (N 1352'41.6", E 99 55'50.0”, Altitude $14 \mathrm{~m}$ ); C63 = Pond at Silpakorn University, Muang District, Nakhonpathom Province (SUT0109063) (N 1348'84.2”, E 10002'64.5”, Altitude $11 \mathrm{~m}$ ); C64 = Bung Bo Ra Ped Lake, Muang District, Nakhon Sawan province (SUT0109064) (N 1542'3.06”, E 100¹0'28.1', Altitude $17 \mathrm{~m}$ ); C65 = Bang Ta nai Canal, Pak Kred District, Nontaburi Province (SUT0109065) (N 1357'10.1”, E 100²9’05.4”, Altitude $15 \mathrm{~m}$ ); C66 = Ratniyom Canal, Sai Noi District, Nontaburi Province (SUT0109066) (N 1404'17.8", E 100¹9'23.7', Altitude 9 m); C67 = Pra Udom Canal, Lad Loom Kaew District, Pathumtani Province (SUT0109067) (N 1401'31.0”, E 100²2’01.1”, Altitude $14 \mathrm{~m}$ ); C68 = Na Mai Canal, Lad Loom Kaew District, Pathumtani Province (SUT0109068) (N 1403'32.7', E 100²6'54.6”, Altitude 22 m); C69 = Wat Ko Phai, Bang Ban District, Ayutthaya Province (SUT0109069) (N 14²4'40.9”, E 100²6'44.6”, Altitude $9 \mathrm{~m}$ ); C70 = Pond at Ban Ta Woong, Ta Woong District, Lopburi Province (SUT0109070) (N 1450’24.4”, E 100²8'21.4”, Altitude $13 \mathrm{~m}$ ); C71 = Suan Ma Dua Waterfall, Pattana Nikom District, Lopburi Province (SUT0109071) (N 1455'06.0”, E 101¹3'09.2”, Altitude $125 \mathrm{~m}$ ); C72 = Pasak Chonlasit Reservior, Pattana Nikom District, Lopburi Province (SUT0109072) (N 1456’22.2”, E 10104'47.4”, Altitude 44 m); C73 = Tam Ru Canal, Muang District, Samut Prakan Province (SUT0109073) (N 13³0'54.7’, E 10041'12.0”, Altitude $1 \mathrm{~m}$ ); C74 = Prachachomchuen Canal, Ampawa District, Sanut Songkram Province (SUT0109074) (N 1325'06.7', E 9957'17.8', Altitude $4 \mathrm{~m}$ ); C75 = Don Ko Canal, Ban Phaew District, Samut Sakhon Province (SUT0109075) (N 13³8'08.0', E 10005'03.0”, Altitude $7 \mathrm{~m}) ; \mathrm{C} 76=$ Ta Pa Canal, Ban Phaew District, Samut Sakhon Province (SUT0109076) (N 13³8'07.3”, E 10006'20.1', Altitude $30 \mathrm{~m}$ ); C77 = Muak Lek Waterfall, Muak Lek District, Saraburi Province (SUT0109077) (N 1443'13.2”, E 101¹1'19.4”, Altitude $156 \mathrm{~m}$ ); C78 = Dong Phya Yen Waterfall, Muak Lek District, Saraburi Province (SUT0109078) (N 1444'0.6”, E 101'11'44.6", Altitude 162 m); C79 $=$ Site 1 at Ched Kot Waterfall, Kaeng Koi District, Saraburi Province (SUT0109079) (N 14²8'48.5”, E $101^{\circ} 10^{\prime} 22.3$ ', Altitude $\left.185 \mathrm{~m}\right)$; C80 = Site2 at Ched Kot Waterfall, Kaeng Koi District, Saraburi Province (SUT0109080) (N 14²8’34.6”, E 101¹0'16.4”, Altitude $157 \mathrm{~m}) ; \mathrm{C} 81=$ Reservoir at Sam Lan Waterfall, Muang District, Saraburi Province (SUT0109081) (N 14²5'52.2”, E 10057'49.6”, Altitude $88 \mathrm{~m}$ ); C82 = Muang Moo Pond, Muang District, Singhaburi Province (SUT0109082) (N 1452'09.1', E 100²4'59.1”, Altitude $16 \mathrm{~m}) ; \mathrm{C} 83$ = Ban Bang Mae Mai Pond, Bang Pla Ma District, Suphanburi Province (SUT0109083) (N 14²0'32.2”, E 10009'04.9”, Altitude 8 m); C84 = Wat bang Yai Pond, Bang Pla Ma District, Suphanburi Province (SUT0109084) (N 14¹8'41.2”, E 10009'03.7”, Altitude $5 \mathrm{~m}$ ); C85 = Bung Cha Wak, Derm Bang Nang Buad District, Supahanburi Province (SUT0109085) (N 1454'04.4”, E 10003'48.0”, Altitude 26 m); C86 = Huay Po Canal, Muang District, Angthong Province (SUT0109086) (N 14³6'08.3”, E 100²4'12.9”, Altitude $14 \mathrm{~m}$ ).

The South: S87 = Water Gate km. 19+500, ChaAm District, Petchaburi Province (SUT0109087) (N 1251'15.1', E 99॰59'48.5”, Altitude $17 \mathrm{~m})$; S88 = Emergency Water Gate, Cha-Am District, Petchaburi Province (SUT0109088) (N 1257.4’26.0”, E $100^{\circ} 02^{\prime}$ 07.5", Altitude $\left.14 \mathrm{~m}\right)$; S89 = Petchaburi Dam, Tayang District, Petchaburi Province (SUT0109089) (N 1254'58.6”, E 9951'34.4”, Altitude 20 m); S90 $=$ Pond at Silpakorn University Petchaburi Campus, Cha-Am district, Petchaburi Province (SUT0109090) (N 11²6'04.6”, E 99²6'56.9”, Altitude 90 m); S91 = Huai Yang stream, Huai Yang District, Prachuabkirikhun Province (SUT0109091) (N 11³6'47.0”, E 9940'08.4", Altitude $18 \mathrm{~m}$ ); S92 = Thap Sakae Stream, Tuap Sakae District, Prachuabkirikhun Province (SUT0109092) (N 11²9’40.1”, E 99³6’20.3”, Altitude $13 \mathrm{~m}$ ); S93 = Kha On Waterfall, Bangsapan District, Prachuabkirikhun Province (SUT0109093) (N 11²6’04.6”E 99²6'56.9”, Altitude 90 m ); S94 = Sai Khu Waterfall, Bangsapan District, Prachuabkirikhun Province (SUT0109094) (N 11¹4’21.8”, E 99²1'36.1", Altitude $83 \mathrm{~m}$ ); S95 = Kapoh Waterfall, Ta Sae District, Chumporn Province (SUT0109095) (N 1044'28.7', E 99¹2'53.9”, Altitude 69 m); S96 $=\mathrm{Ra}$ Canal, Lungsuan District, Chumporn Province (SUT0109096) (N 0959'04.3”, E 9900'59.8”, Altitude $44 \mathrm{~m})$; S97 = Si Kheed Waterfall, Sichon District, Nakhon Sri Thammarat Province (SUT0109097) (N 0900'40.8”, E 9946’30.1”, Altitude 45 m); S98 = Krung Ching Waterfall, Noppitum District, Nakhon Sri Thammarat Province (SUT0109098) (N 0843'14.0”, E 9940'15.2”, Altitude 45 m); S99 = Yod Leung Stream, Noppitum District, Nakhon Sri Thammarat Province (SUT0109099) (N 08³8'10.5”, E 9945'11.6”, Altitude $68 \mathrm{~m}) ; \mathrm{S} 100=$ Palian River, Yan Ta Khao District, Trang Province (SUT0109100) (N 07²2'11.5”, E 9940'51.6", Altitude $12 \mathrm{~m}$ ); S101 = Palian Dam, Palien District, Trang Province (SUT0109101) (N 07¹9'13.2”, E 9948'28.8”, Altitude $43 \mathrm{~m}$ ); S102 = Tone Tok Waterfall, Palien District, Trang Province (SUT0109102) (N 07¹6’44.2”E 9953'10.6”, Altitude $41 \mathrm{~m}) ; \mathrm{S} 103$ = Tone Plew Waterfall, Nayong District, Trang Province (SUT0109103) (N 07³2’48.2”'E 9947'17.0", Altitude $63 \mathrm{~m}$ ); S104 = Ang Thong Waterfall, Sikao District, Trang Province (SUT0109104) (N 07³3'01.2”, E 99²4'56.9”, Altitude 37 m); S105 = Falan Waterfall, Srinakarin District, Pattaloong Province (SUT0109105) (N 07³6’25.9”, E 9954’39.0”, Altitude $80 \mathrm{~m}$ ); S106 = Ban Au Rua Stream, Muang District, Ratchaburi Province (SUT0109106) (N 
13³1'21.9”, E 9950'57.3”, Altitude $152 \mathrm{~m}$ ); S107 = Huay Haeng Stream, Suanphueng District, Ratchaburi Province (SUT0109107) (N 13³1'03.9”, E 99²0'29.2”, Altitude $113 \mathrm{~m}$ ); S108 = Huay Nuang Stream, Suanphueng District, Ratchaburi Province (SUT0109108) (N 13³1'21.9”, E 99¹7’36.5”, Altitude $151 \mathrm{~m})$; S109 = Bangborn Stream, Kraburi District, Ranong Province (SUT0109109) (N 10²0'10.8”, E 9846'48.7', Altitude $18 \mathrm{~m}$ ); S110 = Na Ca Stream, Wild Life Sancuatuary, Muang District, Ranong Province (SUT0109110) (N 9²7'26.6”E 98³0’36.9”, Altitude $3 \mathrm{~m}$ ); S111 = Wiphawadee Waterfall, Donsak District, Surat Thani Province (SUT0109111) (N 98'9.6", E 9940'31.2", Altitude 10 m); S112 = Yan Canal, Wipawadee District, Surathani Province (SUT0109112) (N 9¹2'12.8”, E 9857'20.3”Altitude $66 \mathrm{~m})$; S113 = Ton Sai Waterfall, Tha Lang District, Phuket Province (SUT0109113) (N 8¹’32.4”, E 98²1'58.8’, Altitude $45 \mathrm{~m})$; S114 = Bang Pae Waterfall, Tha Lang District, Phuket Province (SUT0109114) (N 8²'20.5”, E 98²3'49.3', Altitude $50 \mathrm{~m}$ ); S115 = Kathu Waterfall, Kathu District, Phuket Province (SUT0109115) (N 755'49.4”, E 98¹9'34”, Altitude 43 m); S116 = Raman Waterfall, Ta Kua Tung District, Phang Nga
Province (SUT0109116) (N 8²7'8.5”, E 98²8'0.9”, Altitude $33 \mathrm{~m}$ ) ; S117 = Sa Morakot Stream, Klong Tom District, Krabi Province (SUT0309117) (N 755'14.9”, E 99¹5'47.1”, Altitude 24 m); S118 = Panan Waterfall, Kuan galung District, Satoon Province (SUT0109118) (N 651'22.8”, E 1009'48.6”, Altitude $47 \mathrm{~m}$ ); S119= Tha Phae Dam, Kuan Done District, Satoon Province (SUT0109119) (N 649'26”, E 100²’2.3”, Altitude $41 \mathrm{~m}) ; \mathrm{S} 120=$ Klong Muang, Kuan Niang District, Songkhla Province (SUT0109120) (N 7¹2’24.5”, E $100^{\circ} 22^{\prime} 43.1$ ', Altitude $13 \mathrm{~m}$ ).

\section{Habitat at the study sites in Thailand}

The study sites were usually found to be covered with big and medium trees that allow the sunlight to pass through to the stream. The average light intensity was $>10,000$ lux at noon. The current was swift in the rainy season, and water temperature was $21-28^{\circ} \mathrm{C}$. There were small to medium sized rocks all over the streams. The collected snails were found on the rocks, rough sand, and on aquatic plants. The physico-chemical quality of the environment and the water changed with the seasons and affected the study areas during the dry and flood season.

Table 2. Distribution of cercariae obtained from Melanoides tuberculata (32,026 snails) in Thailand. Abbreviations: N - North; NE - Northeast; E - East; C - Central; S - South.

\begin{tabular}{|c|c|c|c|c|c|c|c|}
\hline \multirow[t]{2}{*}{ Cercaria species } & \multicolumn{5}{|c|}{ No. infected snails distribution } & \multirow[t]{2}{*}{ Total } & \multirow{2}{*}{$\begin{array}{l}\text { Infection } \\
\text { rates (\%) }\end{array}$} \\
\hline & $\mathbf{N}=15$ & NE $=20$ & $E=11$ & $C=39$ & $S=35$ & & \\
\hline \multicolumn{8}{|c|}{ Type 1. Parapleurophocercous cercariae: } \\
\hline 1. Haplorchis pumilio & 23 & 0 & 58 & 265 & 25 & 371 & 1.16 \\
\hline 2. Haplorchis taichui & 1 & 2 & 0 & 0 & 89 & 92 & 0.29 \\
\hline 3. Stictodora tridactyla & 0 & 582 & 75 & 210 & 1,315 & 2,182 & 6.81 \\
\hline \multicolumn{8}{|l|}{ Type 2. Pleurophocercous cercariae: } \\
\hline 4. Centrocestus formosanus & 55 & 0 & 3 & 10 & 6 & 74 & 0.23 \\
\hline \multicolumn{8}{|l|}{ Type 3. Xiphidiocercariae: } \\
\hline 5. Acanthatrium hitaense & 9 & 14 & 54 & 1 & 10 & 88 & 0.27 \\
\hline 6. Loxogenoides bicolor & 29 & 802 & 485 & 573 & 484 & 2,373 & 7.41 \\
\hline 7. Haematoloechus similis & 53 & 314 & 92 & 1 & 8 & 468 & 1.46 \\
\hline \multicolumn{8}{|l|}{ Type 4. Megalurous cercariae: } \\
\hline 8. Cloacitrema philippinum & 1 & 0 & 11 & 0 & 0 & 12 & 0.04 \\
\hline 9. Philophthalmus sp. & 0 & 0 & 0 & 52 & 5 & 57 & 0.18 \\
\hline \multicolumn{8}{|l|}{ Type 5. Furcocercous cercariae: } \\
\hline 10. Cardicola alseae & 0 & 2 & 0 & 2 & 22 & 33 & 0.1 \\
\hline 11. Alaria mustelae & 0 & 1 & 2 & 22 & 22 & 47 & 0.15 \\
\hline 12. Transversotrema laruei & 8 & 0 & 21 & 2 & 58 & 89 & 0.28 \\
\hline 13. Apatemon gracilis & 0 & 20 & 0 & 4 & 31 & 55 & 0.17 \\
\hline 14. Mesostephanus appendiculatus & 0 & 3 & 0 & 0 & 0 & 3 & 0.009 \\
\hline \multicolumn{8}{|l|}{ Type 6. Echinostome cercariae: } \\
\hline 15. Echinochasmus pelecani & 0 & 0 & 19 & 0 & 0 & 19 & 0.06 \\
\hline \multicolumn{8}{|l|}{ Type 7. Amphistome cercariae: } \\
\hline 16. Gastrothylax crumenifer & 0 & 0 & 4 & 0 & 4 & 8 & 0.02 \\
\hline \multicolumn{8}{|l|}{ Type 8. Renicolid cercariae: } \\
\hline 17. Cercaria caribbea LXVIII & 0 & 0 & 33 & 12 & 0 & 45 & 0.14 \\
\hline \multicolumn{8}{|c|}{ Type 9. Cotylomicrocercous cercariae: } \\
\hline 18. Podocotyle (Podocotyle) lepomis & 0 & 0 & 0 & 3 & 0 & 3 & 0.009 \\
\hline No. infected snails & 179 & 1,740 & 857 & 1,164 & 2,079 & 6,019 & 18.79 \\
\hline No. of cercaria species & 8 & 9 & 12 & 13 & 13 & 18 & \\
\hline
\end{tabular}




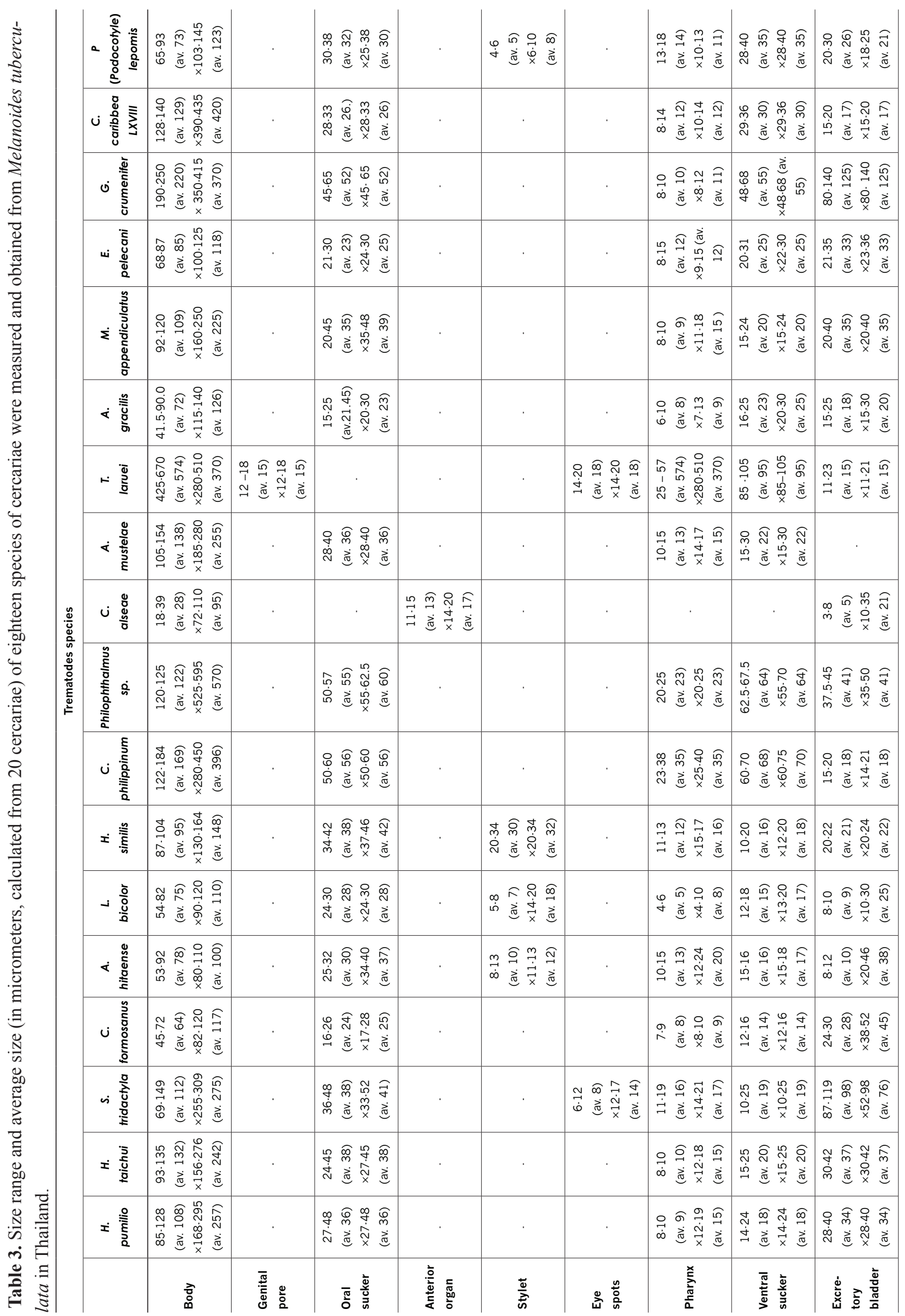




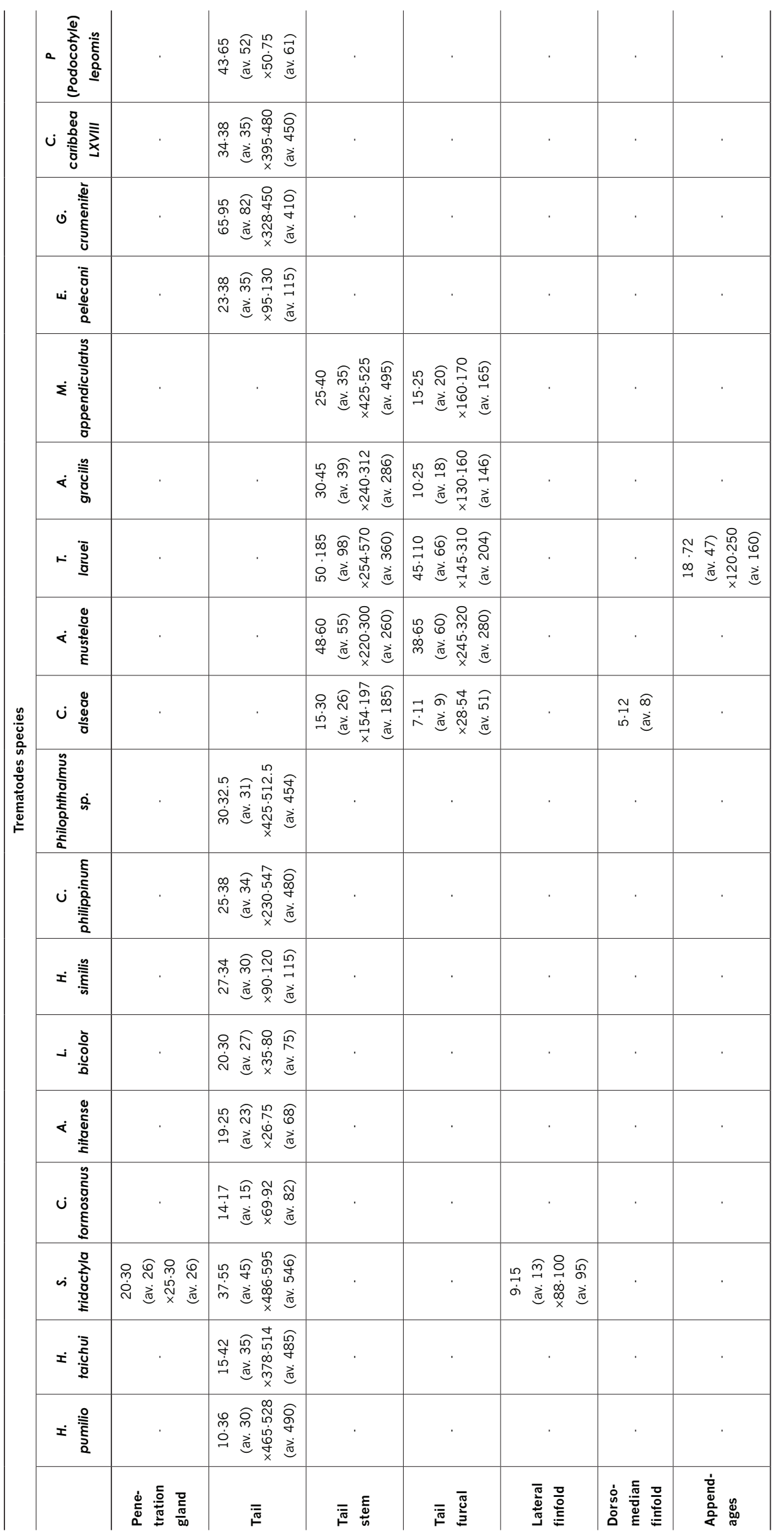




\section{Parasitic infections}

A total of 32,026 Melanoides tuberculata were collected and examined for trematode infections (Fig. 2). The cercarial infections were examined using shedding and crushing methods. The infection rate was $18.79 \%$ $(6,019 / 32,026)$. Nine types and eighteen species of cercariae were categorized. They were (1) Parapleurophocercous cercariae: Haplorchis pumilio, Haplorchis taichui and Stictodora tridactyla; (2) Pleurophocercous cercariae: Centrocestus formosanus; (3) Xiphidiocercariae: Acanthatrium hitaense, Loxogenoides bicolor and Haematoloechus similis; (4) Megalurous cercariae: Cloacitrema philippinum and Philophthalmus sp.; (5) Furcocercous cercariae: Cardicola alseae, Alaria mustelae, Transversotrema laruei, Apatemon gracilis and Mesostephanus appendiculatus; (6) Echinostome cercariae: Echinochasmus pelecani; (7) Amphistome cercariae: Gastrothylax crumenifer; (8) Renicolid cercariae: Cercaria caribbea LXVIII; and, (9) Cotylomicrocercous cercariae: Podocotyle (Podocotyle) lepomis (Table 2).

Characteristics of cercariae were described from living cercariae, fixed cercariae and cercarial images from scanning microscope. Sizes of cercariae were measured for identification of cercarial species (Table 3). The behavior of cercariae was studied and reported for the physiological data of trematodes.

\section{Type 1. Parapleurophocercous cercariae}

\section{Haplorchis pumilio Looss, 1899 (Yamaguti, 1975)}

Haplorchis pumilio (Fig. 3) were found from $371 \mathrm{M}$. tuberculata. The infection rate was $1.16 \%(371 / 32,026)$ (Table 2). The body shape is oval, and its surface is covered with fine spines and sensory hairs. The pigment eyespots and pharynx are present. There are seven pairs of penetration glands, which are arranged in two longitudinal series with a ventral sucker and genital primordia. Their ducts are arranged in two bundles. Four of them were open through the dorsal wall, and four through the ventral wall of the oral sucker in two oblique symmetrical rows. The mouth aperture has transverse rows of spines. The ventral sucker and genital primordia are prevesicular. The excretory bladder has a rounded shape and is composed of fine pigments. No flame cells were found in the tail stem. The tail is long, attached to the dorsal end of the body, with lateral finfolds nearby and a dorso-ventral finfold for the greater distal portion. Cercariae were produced within rediae.

Size range and average size (in micrometers, calculated from 20 cercariae):

Body:

85-128 $\mu \mathrm{m}($ av. $108 \mu \mathrm{m}) \times 168-295$ $\mu \mathrm{m}($ av. $257 \mu \mathrm{m})$

Oral sucker: $27-48 \mu \mathrm{m}($ av. $36 \mu \mathrm{m}) \times 27-48 \mu \mathrm{m}$ (av. $36 \mu \mathrm{m}$ )
Ventral sucker:

14-24 $\mu \mathrm{m}($ av. $18 \mu \mathrm{m}) \times 14-24 \mu \mathrm{m}$ (av. $18 \mu \mathrm{m}$ )

Pharynx: 8-10 $\mu \mathrm{m}($ av. $9 \mu \mathrm{m}) \times 12-19 \mu \mathrm{m}($ av. $15 \mu \mathrm{m})$

Excretory bladder: $28-40 \mu \mathrm{m}($ av. $34 \mu \mathrm{m}) \times 28-40 \mu \mathrm{m}$ (av. $34 \mu \mathrm{m}$ )

Tail: 10-36 $\mu \mathrm{m}($ av. $30 \mu \mathrm{m}) \times 465-528$ $\mu \mathrm{m}($ av. $490 \mu \mathrm{m})$

Movement behavior: The cercaria moved by rolling up and springing the body back to move forward in a screwing motion for 2-4 seconds and then rested for $15-20 \mathrm{sec}-$ onds on the surface of water. It survived up to 2-3 hours in the water after emergence.
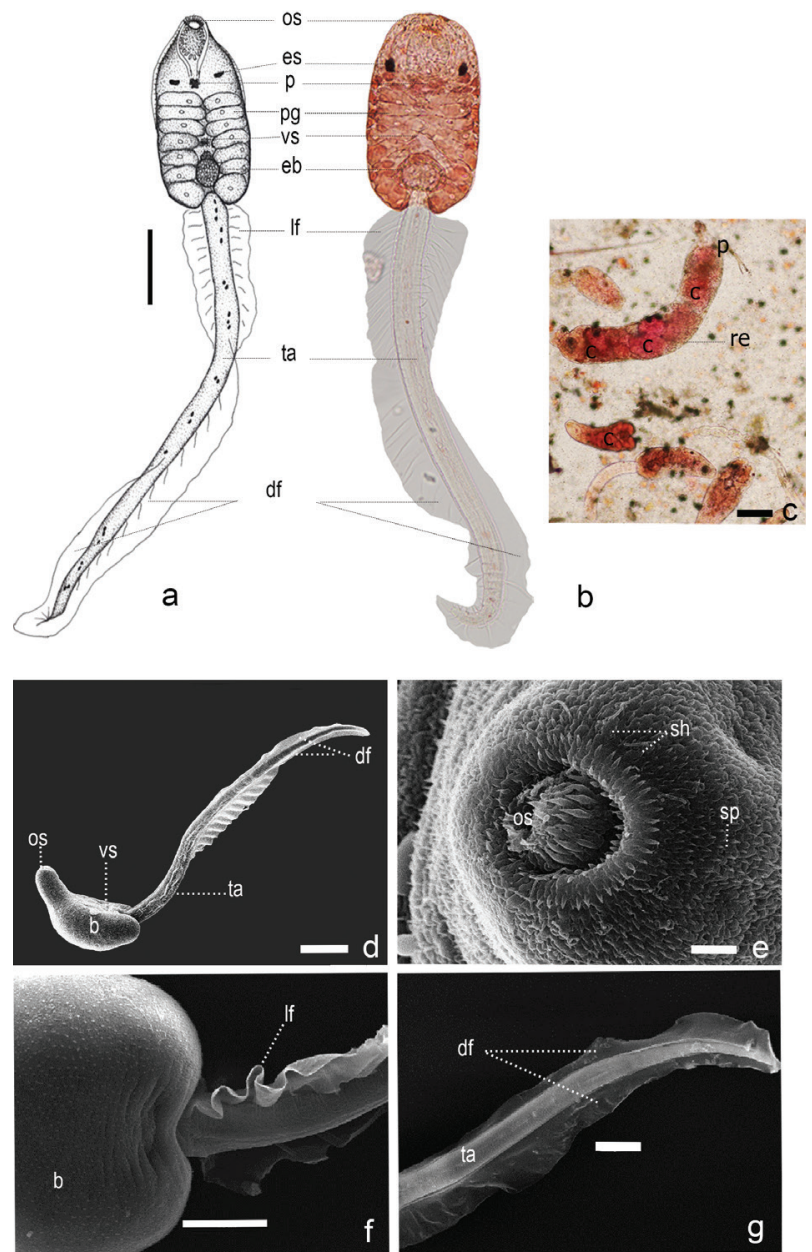

Figure 3. Image of Haplorchis pumilio;

a. Drawing of cercaria structure

b. Cercaria stained with $0.5 \%$ neutral red

c. Redia stained with $0.5 \%$ neutral red

d.- g. Images of Scanning Electronmicroscope

Abbreviations: os - oral sucker, es - eye spot, p - pharynx, pg - penetration gland, vs - ventral sucker, eb - excretory bladder, lf - lateral finfold, ta - tail, df - dorsal finfold, re - redia, c - cercaria, $\mathrm{b}$ - body, sp - spine, sh - sensory hair (scale a, $\mathrm{b}=100 \mu \mathrm{m}$, $\mathrm{c}=10 \mu \mathrm{m})$. 


\section{Haplorchis taichui Nishigori, 1924 (Yamaguti, 1975)}

Haplorchis taichui (Fig. 4) were found from 92 M. tuberculata, the infection rate was $0.29 \%(92 / 32,026)$ (Table 2). Cercarial body is oval in shape, colored with orange yellow, and entirely covered with minute spines and sensory hairs. The oral sucker is situated ventrally in the head region. There are transverse rows of spines at the mouth aperture. The pigment eyespots and a pharynx are present. Seven pairs of penetration glands extend from the pharynx to the end of the body. There are two longitudinal rows with a ventral sucker and genital primordia; their ducts open on the anterior end of the body. The excretory bladder has a round shape and was composed of fine pigments. A long tail is attached to the dorsal end of
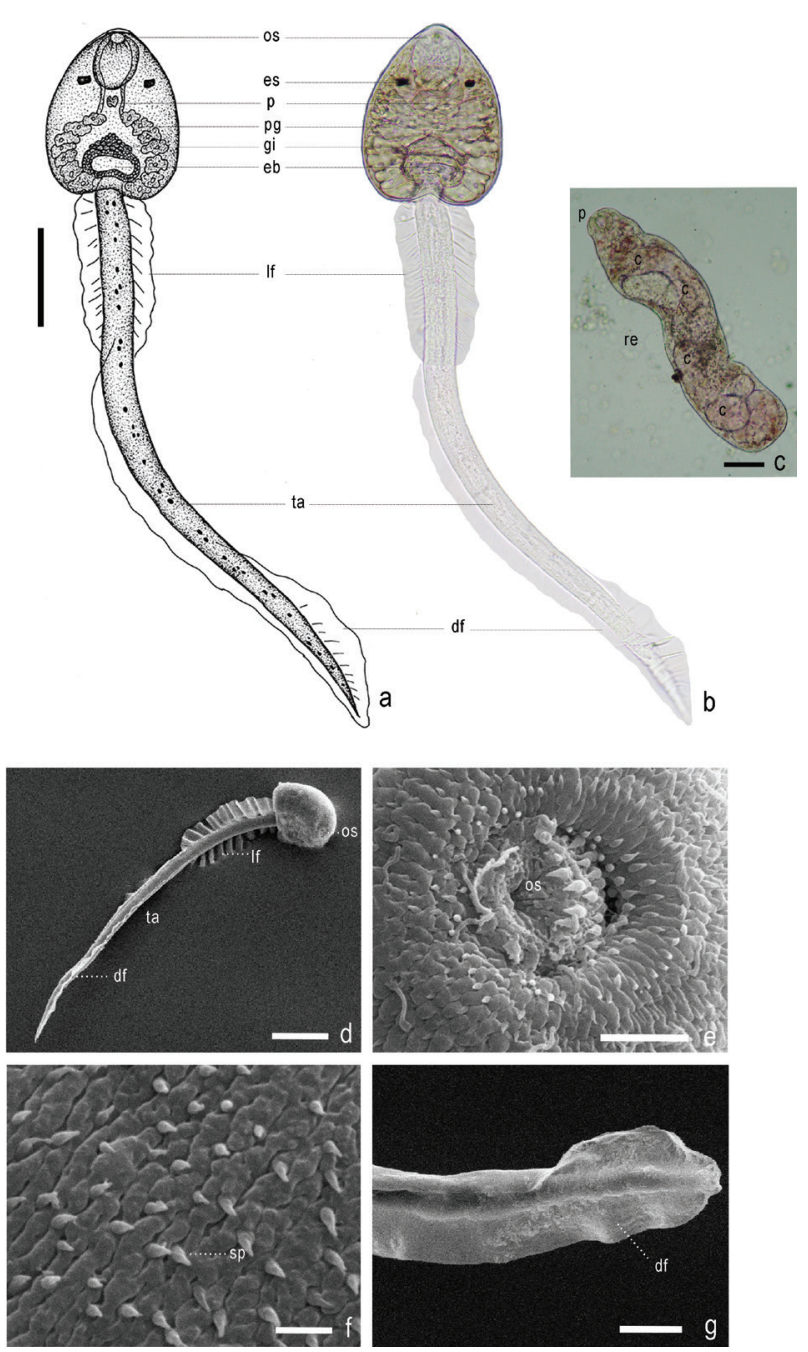

Figure 4. Image of Haplorchis taichui;

a. Drawing of cercaria structure

b. Cercaria without staining

c. Redia without staining

d.- g. Images of Scanning Electronmicroscope

Abbreviations: os - oral sucker, es - eye spot, p - pharynx, pg penetration gland, gi - genital primordial, eb - excretory bladder, lf - lateral finfold, ta - tail, df - dorsal finfold, re - redia, c - cercaria, sp - spine (scale a, $\mathrm{b}=100 \mu \mathrm{m}, \mathrm{c}=20 \mu \mathrm{m}$ ). the body, with lateral finfolds nearby and a dorso-ventral finfold for the greater distal portion. No flame cells are found in the tail stem. Cercariae were produced within the rediae.

Size range and average size (in micrometers, calculated from 20 cercariae):

Body:

93-135 $\mu \mathrm{m}($ av. $132 \mu \mathrm{m}) \times 156-276$

Oral sucker: $\quad 24-45 \mu \mathrm{m}($ av. $38 \mu \mathrm{m}) \times 27-45 \mu \mathrm{m}$ $\mu \mathrm{m}$ (av. $242 \mu \mathrm{m})$ (av. $38 \mu \mathrm{m}$ )

Ventral sucker: $\quad 15-25 \mu \mathrm{m}$ (av. $20 \mu \mathrm{m}) \times 15-25 \mu \mathrm{m}$ (av. $20 \mu \mathrm{m}$ )

Pharynx: $\quad 8-10 \mu \mathrm{m}($ av. $10 \mu \mathrm{m}) \times 12-18 \mu \mathrm{m}$ (av. $15 \mu \mathrm{m})$

Excretory bladder: $\quad 30-42 \mu \mathrm{m}$ (av. $37 \mu \mathrm{m}) \times 30-42 \mu \mathrm{m}$ (av. $37 \mu \mathrm{m}$ )

Tail:

$15-42 \mu \mathrm{m}($ av. $35 \mu \mathrm{m}) \times 378-514$ $\mu \mathrm{m}$ (av. $485 \mu \mathrm{m})$

Movement behavior: Cercariae were escaped from the rediae. In the water, they floated on the surface or in the middle. The body part sank lower than the tail. The movement rolled around fast on the water, about 8 to 12 seconds, then rested for 5-10 seconds. It survived up to 2-3 hours in the water after emergence.

\section{Stictodora tridactyla Martin \& Kuntz, 1955 (Yama- guti, 1975)}

Stictodora tridactyla (Fig. 5) were found from 2,182 M. tuberculata. The infection rate was $6.81 \%(2,182 / 32,026)$ (Table 2). The body is oval in shape and yellowish brown in color. There are 3 rows of oral spines $(4-6,12-14$, 22-24), and 7 pairs of penetration glands in 4 groups of $3: 4: 4: 3$. The penetration ducts are open near the oral sucker. The eight ducts are arranged in two bundles, four open through the dorsal wall, and four through the ventral wall. The ventral sucker is small. There is one pair of eye spots with coarse granules, with a small globular pharynx between the eye spots. The excretory bladder in the flattened V-shaped is situated at the end of the body. The tail is longer than the body with a bilaterial finfold and a dorso-ventral finfold. Both the dorsal and ventral finfolds arose at a short distance from the anterior and the posterior end of the lateral finfold. There is no flame cell, but 3-5 groups of pigment, an opening duct of the excretory bladder at the tip of the tail. Cercariae were produced within the rediae.

Size range and average size (in micrometers, calculated from 20 cercariae):

Body:

69-149 $\mu \mathrm{m}($ av. $112 \mu \mathrm{m}) \times 255-309$ $\mu \mathrm{m}$ (av. $275 \mu \mathrm{m})$

Oral sucker: $\quad 36-48 \mu \mathrm{m}($ av. $38 \mu \mathrm{m}) \times 33-52 \mu \mathrm{m}$ (av. $41 \mu \mathrm{m}$ )

Eye spots: $\quad 6-12 \mu \mathrm{m}(\mathrm{av} .8 \mu \mathrm{m}) \times 12-17 \mu \mathrm{m}(\mathrm{av}$. $14 \mu \mathrm{m})$ 
Pharynx:

Ventral sucker:

$11-19 \mu \mathrm{m}($ av. $16 \mu \mathrm{m}) \times 14-21 \mu \mathrm{m}$ (av. $17 \mu \mathrm{m}$ )

$\begin{array}{ll} & \text { (av. } 19 \mu \mathrm{m}) \\ \text { Excretory bladder: } & 87-119 \mu \mathrm{m}(\text { av. } 98 \mu \mathrm{m}) \times 52-98 \mu \mathrm{m}\end{array}$ (av. $76 \mu \mathrm{m}$ )

Penetration gland: $\quad 20-30 \mu \mathrm{m}($ av. $26 \mu \mathrm{m}) \times 25-30 \mu \mathrm{m}$ (av. $26 \mu \mathrm{m}$ )

Tail:

37-55 $\mu \mathrm{m}($ av. $45 \mu \mathrm{m}) \times 486-595$ $\mu \mathrm{m}$ (av. $546 \mu \mathrm{m})$

Lateral finfold: $\quad 9-15 \mu \mathrm{m}($ av. $13 \mu \mathrm{m}) \times 88-100 \mu \mathrm{m}$ (av. 95)

Movement behavior: The cercaria floated on the surface or in the middle of the water.

The body sank lower than the tail. The body moves by turning over left and right. The rolling movement is fast on the water, about 5-7 seconds, and rests for about 25-27 seconds. The body sinks on the surface of the water and then moves upside down.
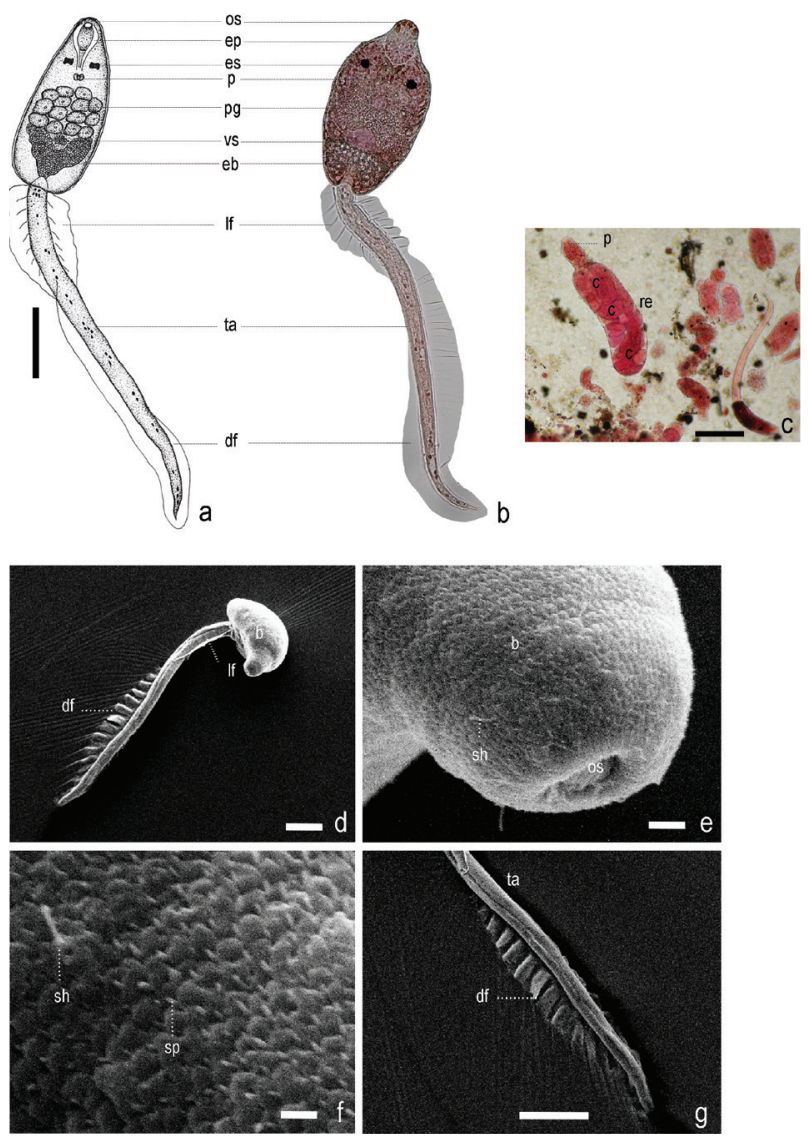

Figure 5. Image of Stictodora tridactyla;

a. Drawing of cercaria structure

b. Cercaria stained with $0.5 \%$ neutral red

c. Redia stained with $0.5 \%$ neutral red

d.- g. Images of Scanning Electronmicroscope

Abbreviations: os - oral sucker, ep - esophagus, es - eye spot, p - pharynx, pg - penetration gland, vs - ventral sucker, eb - excretory bladder, lf - lateral finfold, ta - tail, df - dorsal finfold, re - redia, c - cercaria, b - body, sp - spine, sh - sensory hair (scale $\mathrm{a}, \mathrm{b}=100 \mu \mathrm{m}, \mathrm{c}=10 \mu \mathrm{m})$.

\section{Type 2. Pleurophocercous cercariae}

\section{Centrocestus formosanus Nishigori, 1924 (Yamagu- ti, 1975)}

This parasite (Fig. 6) was found in $74 M$. tuberculata. The infection rate was $0.23 \%(74 / 32,026)$ (Table 2). Cercarial body is oval in shape. A pair of eyespots lay at the level of the pharynx. The oral sucker has two rows of oral spines similar to hooks of the tapeworm (rostellar hooks, four in the anterior and five in the posterior) on the dorsal wall of the mouth aperture. Short esophagus, the parenchymal body is spinulate, and yellowish brown in color. Acetabulum is found between the intestinal bifurcation and the excretory vesical. The bladder is a flattened V-shape. Seven pairs of penetration glands lay anterolateral to the acetabulum in front of an inverted V-shape. Cystogenous cells are distributed in the posterior part. The genital primordial part is somewhat elongated and triangular, between the acetabulum and the excretory vesicle. The tail is slender, with a very indistinct dorsal and ventral finfolds, both of which are more conspicuous in the distal half, with a tiny spike on the tip. Cercariae were produced within the rediae.

Size range and average size (in micrometers, calculated from 20 cercariae):

Body:

45-72 $\mu \mathrm{m}$ (av. $64 \mu \mathrm{m}) \times 82-120 \mu \mathrm{m}$ (av. $117 \mu \mathrm{m})$

Oral sucker: $16-26 \mu \mathrm{m}$ (av. $24 \mu \mathrm{m}) \times 17-28 \mu \mathrm{m}$ (av. $25 \mu \mathrm{m})$

Ventral sucker: $12-16 \mu \mathrm{m}($ av. $14 \mu \mathrm{m}) \times 12-16 \mu \mathrm{m}$ (av. $14 \mu \mathrm{m})$

Pharynx:

7-9 $\mu \mathrm{m}($ av. $8 \mu \mathrm{m}) \times 8-10 \mu \mathrm{m}($ av. 9 $\mu \mathrm{m})$

Excretory bladder: $\quad 24-30 \mu \mathrm{m}($ av. $28 \mu \mathrm{m}) \times 38-52 \mu \mathrm{m}$ (av. $45 \mu \mathrm{m})$

Tail:

14-17 $\mu \mathrm{m}($ av. $15 \mu \mathrm{m}) \times 69-92 \mu \mathrm{m}$ (av. $82 \mu \mathrm{m}$ )

Movement behavior: The cercaria moved by rolling up and springing the body back to move forward in a screwing motion for 8-10 seconds and then rested for about 4550 seconds. It survived up to 3-4 hours in the water after emergence.

\section{Type 3. Xiphidiocercariae}

\section{Acanthatrium hitaense Koga, 1953 (Yamaguti, 1975)}

This parasite (Fig. 7) was found in 88 M. tuberculata. The infection rate was $0.27 \%(88 / 32,026)$ (Table 2$)$. The parasite is virgulate xiphidiocercaria. The body is oval in shape and white in color. There are stylet and virgulate glands in the oral sucker, and 2 pairs of penetration glands in each side of the body. The pharynx is round and short, the ventral sucker is smaller than the oral sucker, and, the small excretory bladder is located at the end of the body. The tail 

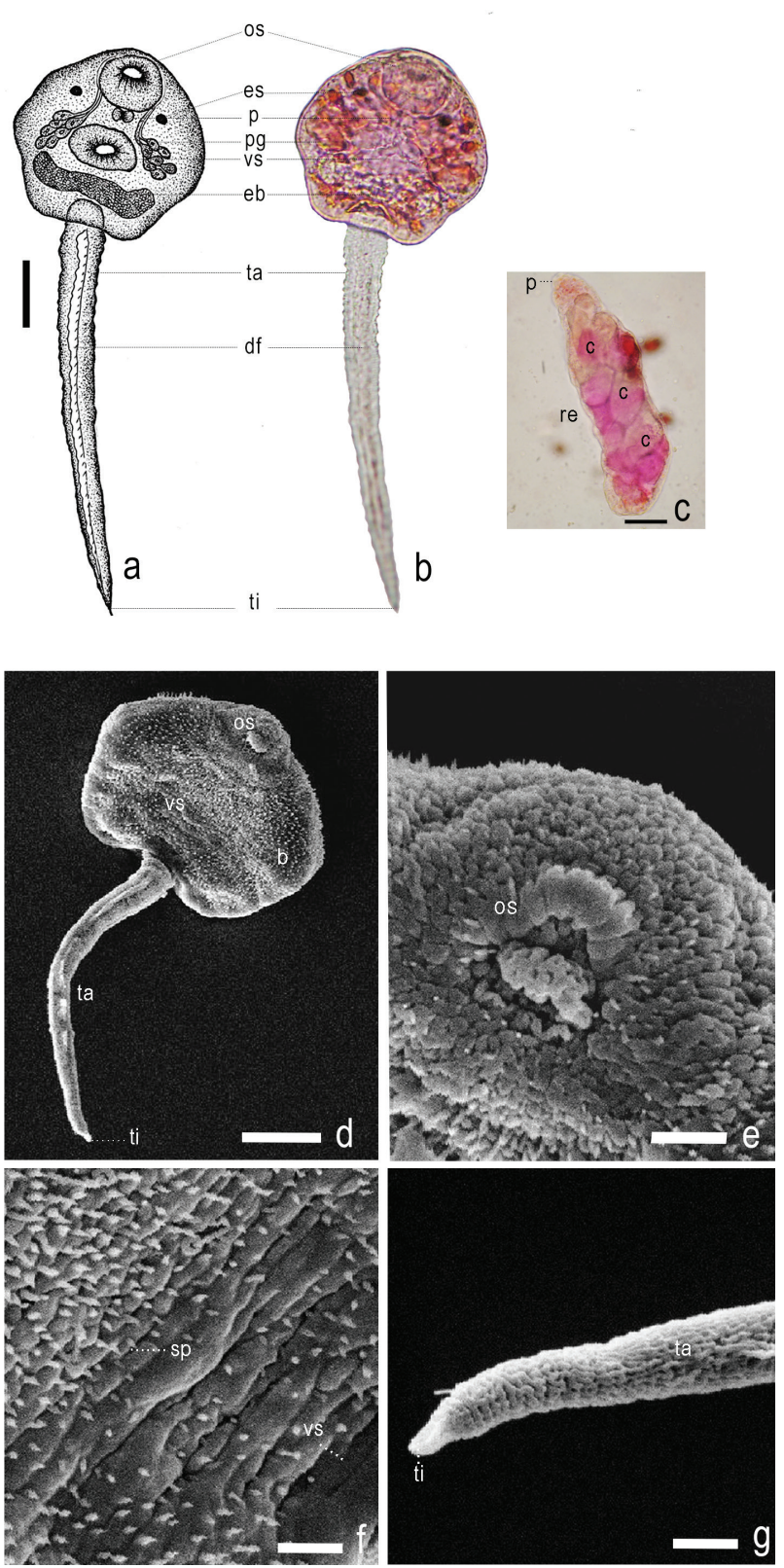

Figure 6. Image of Centrocestus formosanus;

a. Drawing of cercaria structure

b. Cercaria stained with $0.5 \%$ neutral red

c. Redia stained with $0.5 \%$ neutral red

d.- g. Images of Scanning Electronmicroscope

Abbreviations: os - oral sucker, es - eye spot, p - pharynx, pg penetration gland, vs - ventral sucker, eb - excretory bladder, ta - tail, df - dorsal finfold, ti - tip, re - redia, c - cercaria, b - body, sp - spine (scale $\mathrm{a}, \mathrm{b}=100 \mu \mathrm{m}, \mathrm{c}=10 \mu \mathrm{m})$.

is shorter than the body, inserted to the posterior end of the body. Cercariae were produced within the sporocyst.

Size range and average size (in micrometers, calculated from 20 cercariae):

Body:

53-92 $\mu \mathrm{m}$ (av. $78 \mu \mathrm{m}) \times 80-110 \mu \mathrm{m}$ (av. $100 \mu \mathrm{m}$ )
Oral sucker:

Stylet:

Ventral sucker:

Pharynx:

Excretory bladder:

Tail:

Movement behavior: The cercaria floated on the surface or in the middle of the water. They moved by rolling up and springing the body back to move forward in a screwing motion for 45-60 seconds, and then rested for 2-5 seconds at the water surface. Some cercariae were stuck on the surface of the container, and moved by the oral sucker and ventral sucker. It survived up to 2-4 hours in the water after emergence.
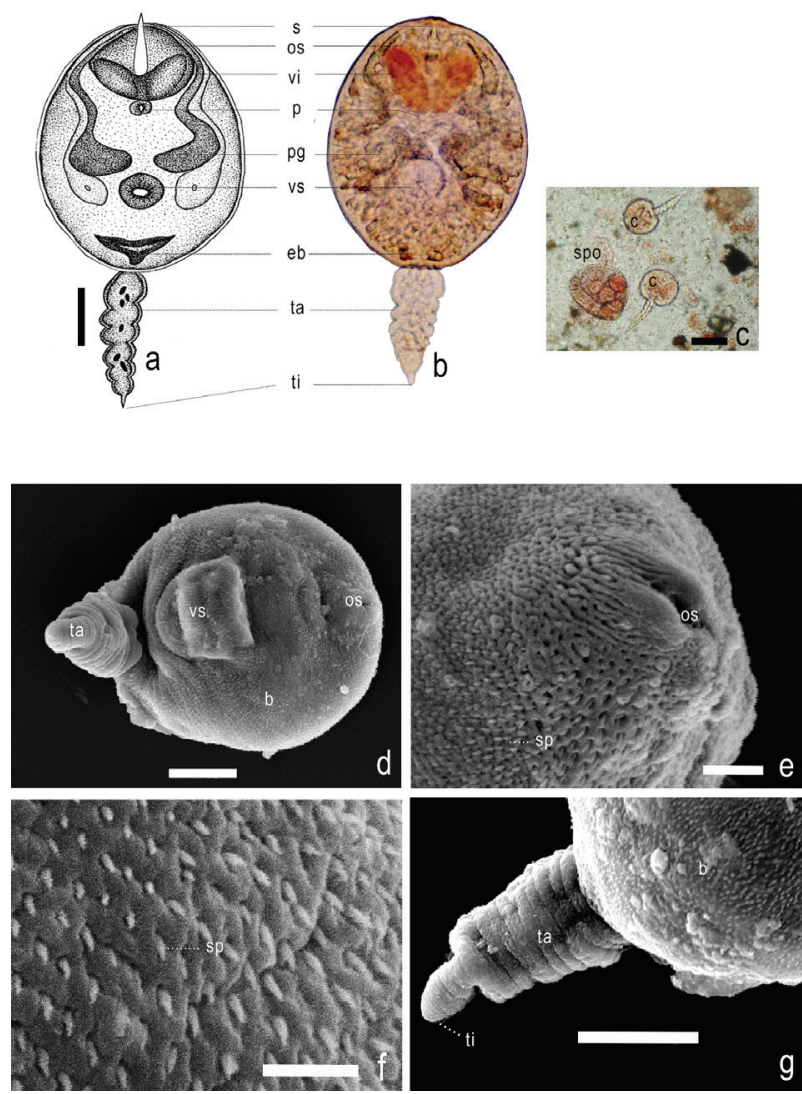

Figure 7. Image of Acanthatrium hitaense;

a. Drawing of cercaria structure

b. Cercaria stained with $0.5 \%$ neutral red

c. Sporocyst stained with $0.5 \%$ neutral red

d.- g. Images of Scanning Electronmicroscope

Abbreviations: s - stylet, os - oral sucker, vi - vigulate gland, p - pharynx, pg - penetration gland, vs - ventral sucker, eb excretory bladder, ta - tail, ti - tip, spo : sporocyst, c - cercaria, $\mathrm{b}$ - body, sp - spine (scale $\mathrm{a}, \mathrm{b}=25 \mu \mathrm{m}, \mathrm{c}=50 \mu \mathrm{m}$ ). 


\section{Loxogenoides bicolor Kaw, 1945 (Yamaguti, 1975)}

Loxogenoides bicolor (Fig. 8) was found from 2,373 $M$. tuberculata. The infection rate was $7.41 \%(2,373 / 32,026)$ (Table 2). The body of cercaria is spinose and oval in shape. Its entire body is dotted with granules. The ventral sucker is smaller than the oral sucker. A virgular organ is located in the region of the oral sucker. A stylet is present. Three pairs of penetration glands exist: two anterior pairs and a posterior pair. The penetration glands had granules and ducts. The ducts opened near the tip of the stylet. There is a C-shaped genital primordium and a U-shaped excretory bladder. The tail is spinose, with slightly longer spines at the tip. Cercariae were produced within the sporocyst.
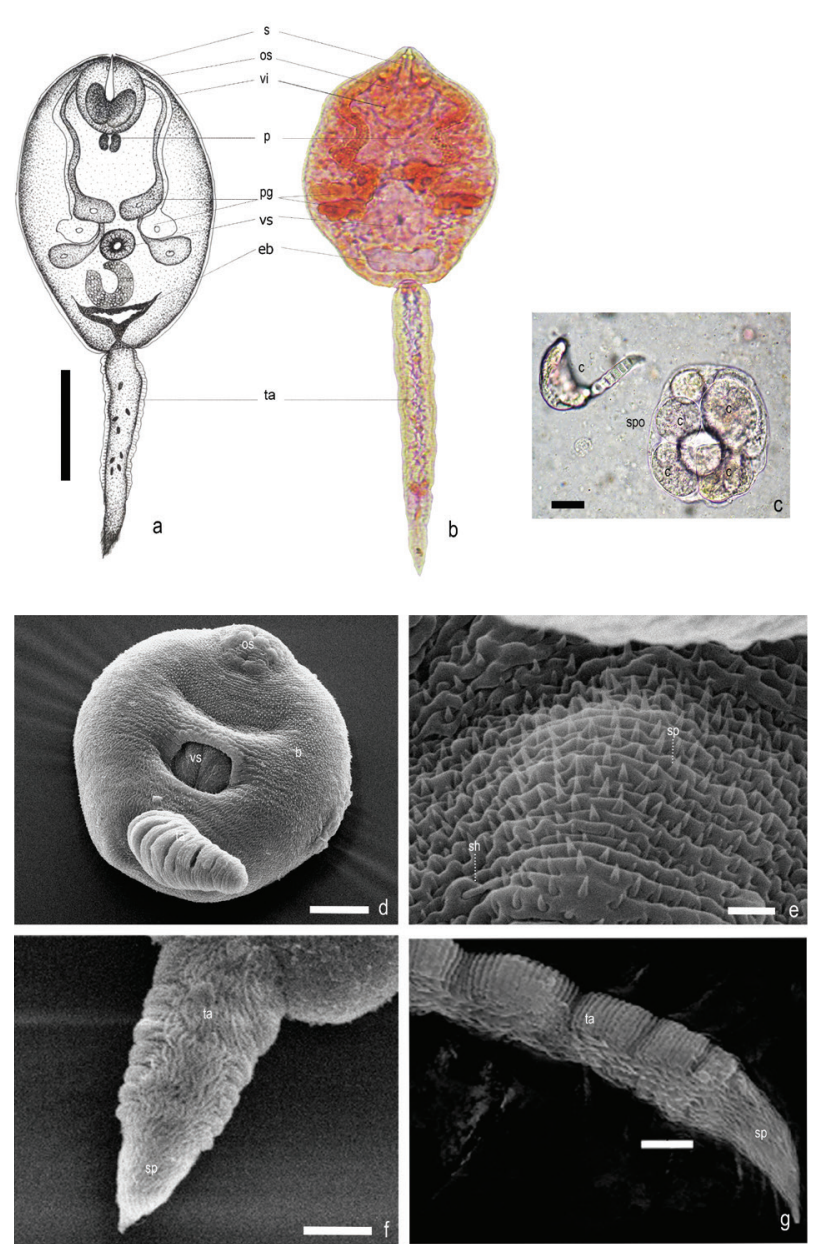

Figure 8. Image of Loxogenoides bicolor;

a. Drawing of cercaria structure

b. Cercaria stained with $0.5 \%$ neutral red

c. Sporocyst stained with $0.5 \%$ neutral red

d.- g. Images of Scanning Electronmicroscope

Abbreviations: s - stylet, os - oral sucker, vi - vigulate gland, p - pharynx, pg - penetration gland, vs - ventral sucker, eb - excretory bladder, ta - tail, spo : sporocyst, c - cercaria, b - body, $\mathrm{sp}$ - spine (scale $\mathrm{a}, \mathrm{b}=50 \mu \mathrm{m}, \mathrm{c}=20 \mu \mathrm{m}$ ).

Size range and average size (in micrometers, calculated from 20 cercariae):

Body:

Oral sucker:

Stylet:

Ventral sucker:

Pharynx:

Excretory bladder: $\quad 8-10 \mu \mathrm{m}($ av. $9 \mu \mathrm{m}) \times 10-30 \mu \mathrm{m}$ (av. $25 \mu \mathrm{m})$

Tail: (av. $75 \mu \mathrm{m}$ )

Movement behavior: The cercaria moved by folding its tail and rolling up the body and moved from left to right quickly. In resting position, they floated on the surface or in the middle of the water. The body sank lower than the tail. The cercaria moved about $60-75$ seconds, and rested for about 2-5 seconds. It survived up to 2-3 hours in the water after emergence.

\section{Haematoloechus similis Looss, 1899 (Yamaguti, 1975)}

Haematoloechus similis (Fig. 9) was found in $468 \mathrm{M}$. tuberculata. The infection rate was $1.46 \%(468 / 32,026)$ (Table 2). This parasite was classified into Xiphidiocercariae. The body is ovate, and the surface is covered with spines. Cytogenous cell is not observed. A stylet is 30-32 $\mu \mathrm{m}$ long, with no virgulate gland. Six pairs of penetration glands of irregular shape are present, extending from the middle of the body to near the posterior end of body, each with large nuclei and fine granules. Their ducts are bundled, one on each side, opening near the tip of the stylet. Prepharynx is short, and pharynx poorly differentiated. Esophagus, ceca and genitalia are not developed. The excretory vesicle is $\mathrm{Y}$ shape. The flame cell formula is $2[(3+3+3)+(3+3+3)]=36$. The tail is not finflod. Cercariae were produced within the sporocyst.

Size range and average size (in micrometers, calculated from 20 cercariae):

Body:

Oral sucker:

Stylet:

Ventral sucker:

Pharynx:

Excretory bladder:
87-104 $\mu \mathrm{m}($ av. $95 \mu \mathrm{m}) \times 130-164$ $\mu \mathrm{m}$ (av. $148 \mu \mathrm{m})$

$34-42 \mu \mathrm{m}($ av. $38 \mu \mathrm{m}) \times 37-46 \mu \mathrm{m}$ (av. $42 \mu \mathrm{m}$ )

20-34 $\mu \mathrm{m}$ (av. $30 \mu \mathrm{m}) \times 20-34 \mu \mathrm{m}$ (av. $32 \mu \mathrm{m}$ )

$10-20 \mu \mathrm{m}$ (av. $16 \mu \mathrm{m}) \times 12-20 \mu \mathrm{m}$ (av. $18 \mu \mathrm{m}$ )

$11-13 \mu \mathrm{m}$ (av. $12 \mu \mathrm{m}) \times 15-17 \mu \mathrm{m}$ (av. $16 \mu \mathrm{m}$ )

20-22 $\mu \mathrm{m}$ (av. $21 \mu \mathrm{m}) \times 20-24 \mu \mathrm{m}$ (av. $22 \mu \mathrm{m}$ ) 

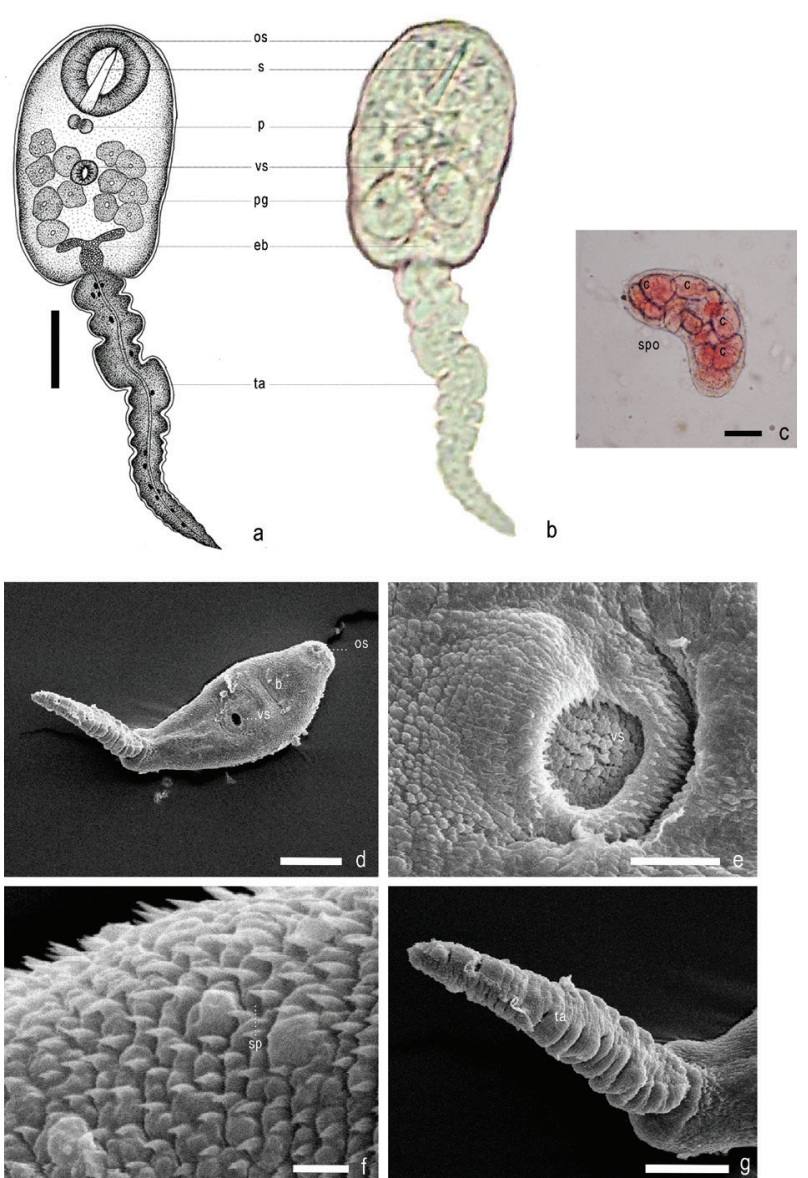

Figure 9. Image of Haematoloechus similis;

a. Drawing of cercaria structure

b. Cercaria without staining

c. Sporocyst stained with $0.5 \%$ neutral red

d.- g. Images of Scanning Electronmicroscope

Abbreviations: s - stylet, os - oral sucker, p - pharynx, pg - penetration gland, vs - ventral sucker, eb - excretory bladder, ta - tail, spo : sporocyst, c - cercaria, $\mathrm{b}$ - body, sp - spine (scale a, $\mathrm{b}=50$ $\mu \mathrm{m}, \mathrm{c}=50 \mu \mathrm{m})$.

Tail:

27-34 $\mu \mathrm{m}$ (av. $30 \mu \mathrm{m}) \times 90-120 \mu \mathrm{m}$ (av. $115 \mu \mathrm{m}$ )

Movement behavior: The cercaria floated on the surface or in the middle of the water. The body sank lower than the tail. It moved by folding its tail back to the body and turning its body to roll quickly from left to right, darting forward for about 15-20 seconds, and resting for about 10-15 seconds. It survived up to 1-2 hours in the water after emergence.

\section{Type 4. Megalurous cercariae}

\section{Cloacitrema philippinum Velasquez, 1969 (Yamagu- ti, 1975)}

Cloacitrema philippinum (Fig. 10) was found in 12 M. tuberculata. The infection rate was $0.04 \%(12 / 32,026)$ (Table 2). The body is elongate and muscular. There is no eye spot, and no spine on the body surface. There are long prepharynx and pharynx. The long ceca runs almost to the end of the body. There are numerous sensory papillae on the surface of body. The oral sucker has 12 opening ducts. The cystogenous cells were found all over the body. The thin wall of the excretory vesicle extends when moving, and the ventral sucker is bigger than the oral sucker. The long tail is inserted to the posterior end of the body. The clear vacuoles distributed along the tail, containing granules. There is an adhesive organ at the tip of the tail, with no lateral finfolds. Cercariae were produced within the rediae.

Size range and average size (in micrometers, calculated from 20 cercariae):

Body:

$122-184 \mu \mathrm{m}($ av. $169 \mu \mathrm{m}) \times 280-450$ $\mu \mathrm{m}($ av. $396 \mu \mathrm{m})$

Oral sucker: $\quad 50-60 \mu \mathrm{m}($ av. $56 \mu \mathrm{m}) \times 50-60 \mu \mathrm{m}$ (av. $56 \mu \mathrm{m})$
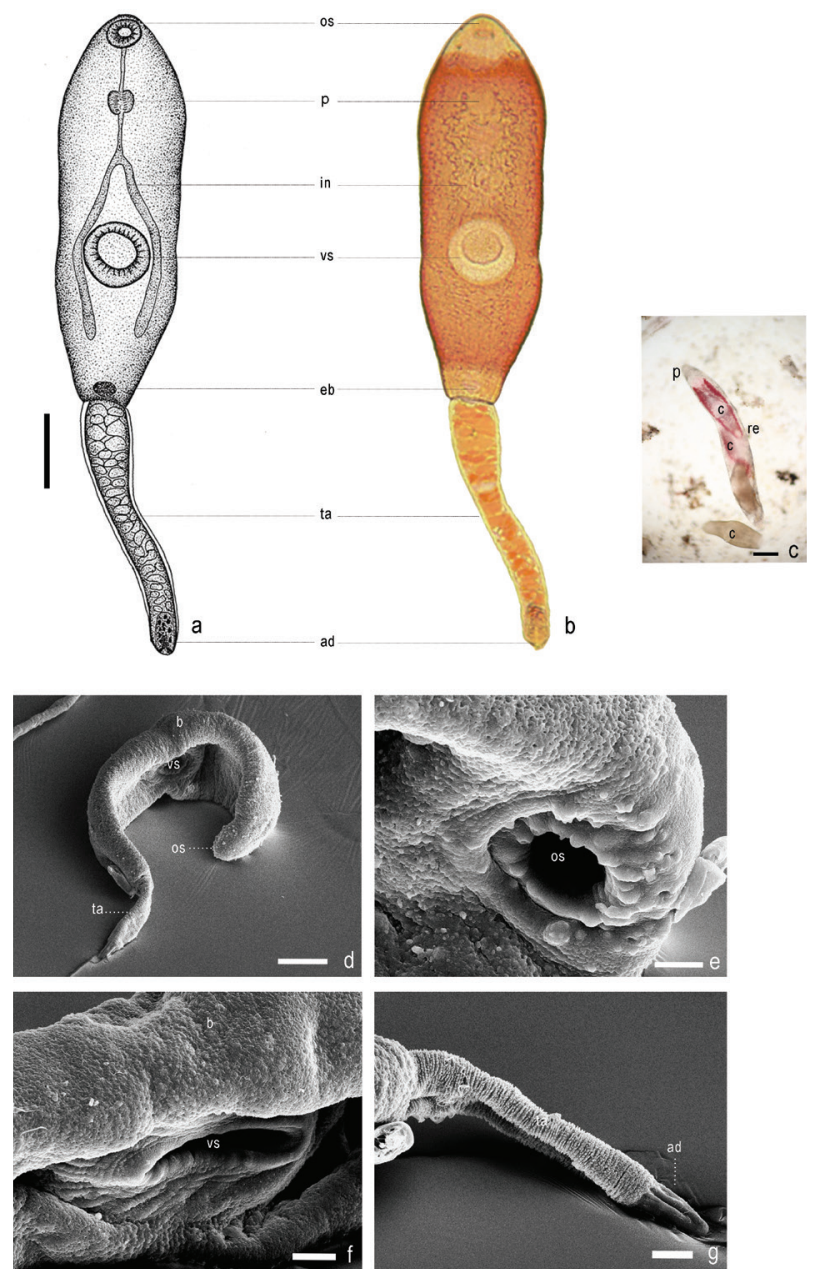

Figure 10. Image of Cloacitrema philippinum;

a. Drawing of cercaria structure

b. Cercaria stained with $0.5 \%$ neutral red

c. Redia stained with $0.5 \%$ neutral red

d.- g. Images of Scanning Electronmicroscope

Abbreviations: os - oral sucker, $\mathrm{p}$ - pharynx, in - intestine, vs ventral sucker, eb - excretory bladder, ta - tail, ad - adhesive gland, re - redia, c - cercaria, b - body(scale a, $\mathrm{b}=100 \mu \mathrm{m}, \mathrm{c}=50 \mu \mathrm{m})$. 
Ventral sucker:

$60-70 \mu \mathrm{m}($ av. $68 \mu \mathrm{m}) \times 60-75 \mu \mathrm{m}$ (av. $70 \mu \mathrm{m})$

Pharynx:

$23-38 \mu \mathrm{m}($ av. $35 \mu \mathrm{m}) \times 25-40 \mu \mathrm{m}$ (av. $35 \mu \mathrm{m}$ )

Excretory bladder: $15-20 \mu \mathrm{m}($ av. $18 \mu \mathrm{m}) \times 14-21 \mu \mathrm{m}$ (av. $18 \mu \mathrm{m})$

Tail:

25-38 $\mu \mathrm{m}$ (av. $34 \mu \mathrm{m}) \times 230-547$ $\mu \mathrm{m}(\mathrm{av} .480 \mu \mathrm{m})$

Movement behavior: The cercaria floated on the surface or in the middle of the water. It moved by stretching the body and rolling on the water surface around 20-28 seconds and resting for about 2-4 seconds. The adhesive gland anchored on the container surface. It survived up to 2-4 hours in the water after emergence.

\section{Philophthalmus sp. Looss, 1899 (Urabe, 2005)}

Philophthalmus sp. (Fig. 11) was found in $57 \mathrm{M}$. tuberculata. The infection rate was $0.18 \%(57 / 32,026)$ (Table 2$)$.
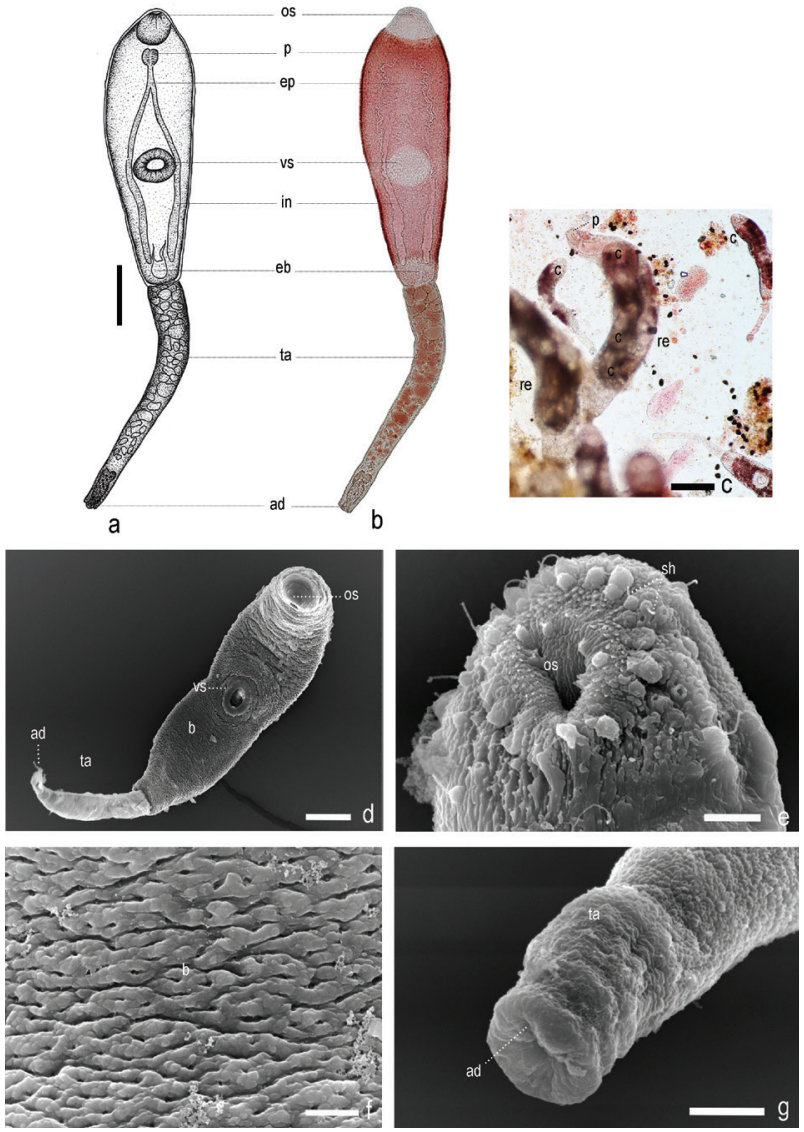

Figure 11. Image of Philopthalmus sp.;

a. Drawing of cercaria structure

b. Cercaria stained with $0.5 \%$ neutral red

c. Redia stained with $0.5 \%$ neutral red

d.- g. Images of Scanning Electronmicroscope

Abbreviations: os - oral sucker, p - pharynx, ep - esophagus, vs - ventral sucker, in - intestine, eb - excretory bladder, ta - tail, ad - adhesive gland, re - redia, c - cercaria, b - body (scale a, b $=100 \mu \mathrm{m}, \mathrm{c}=50 \mu \mathrm{m})$.
The body is elongated and white in color, with numerous minute spines on the half body at the posterior end. It has a muscular body, no eye spot, long prepharynx and pharynx, Y-shaped esophagus, and a large ventral sucker. The intestine runs almost to the end of the body. There are plenty of cyst glands along their bodies. The tail is the same length as the body but flexible, with various sizes of vacuole and granules along the tail. The adhesive gland cells were found. Cercariae were produced within the rediae.

Size range and average size (in micrometers, calculated from 20 cercariae):

Body:

120-125 $\mu \mathrm{m}($ av. $122 \mu \mathrm{m}) \times 525-$ $595 \mu \mathrm{m}($ av. $570 \mu \mathrm{m})$

Oral sucker: $50-57 \mu \mathrm{m}($ av. $55 \mu \mathrm{m}) \times 55-62.5 \mu \mathrm{m}$ (av. $60 \mu \mathrm{m}$ )

Ventral sucker: 62.5-67.5 $\mu \mathrm{m}($ av. $64 \mu \mathrm{m}) \times 55-70$ $\mu \mathrm{m}$ (av. $64 \mu \mathrm{m})$

Pharynx: $20-25 \mu \mathrm{m}$ (av. $23 \mu \mathrm{m}) \times 20-25 \mu \mathrm{m}$ (av. $23 \mu \mathrm{m}$ )

Excretory bladder: $\quad 37.5-45 \mu \mathrm{m}($ av. $41 \mu \mathrm{m}) \times 35-50 \mu \mathrm{m}$ (av. $41 \mu \mathrm{m}$ )

Tail: 30-32.5 $\mu \mathrm{m}$ (av. $31 \mu \mathrm{m}) \times 425-$ $512.5 \mu \mathrm{m}$ (av. $454 \mu \mathrm{m})$

Movement behavior: The cercaria floated on the surface or in the middle of the water. It moved by stretching and floating on the surface of the water, and moved forward. Adhesive gland are stuck on the container for cercarial moving. The cercariae became metacercarial cyst immediately in the container.

\section{Type 5. Furcocercous cercariae}

\section{Cardicola alseae Meade \& Pratt, 1965 (Yamaguti, 1975)}

Cardicola alseae (Fig. 12) were found from 47 M. tuberculata which is equivalent to an infection rate of $0.1 \%$ $(33 / 32,026)$ of the total number of the collected snails (Table 2). The small hook-liked body had an anterior organ, and was covered with minute spines. The longer spines were found in some parts of the body, the dorsal and ventral of the posterior end. The dorso-median finfold was observed in the middle part of the body. Many large granules were observed, with a penetration gland located at the middle part of the body. The excretory bladder was small. The tail is furcocercous; its furcae are shorter than the beginning of the tail, and sharp like animal crawls. Minute spines and sensory hairs were observed. A caudal body, longitudinal muscle, no flame cell, and furcal finfold were observed, with the opening duct of excretory bladder at the fork tail tip. Cercariae were produced within the sporocyst.

Size range and average size (in micrometers, calculated from 20 cercariae):

Body:

$18-39 \mu \mathrm{m}$ (av. $28 \mu \mathrm{m}) \times 72-110 \mu \mathrm{m}$ (av. $95 \mu \mathrm{m}$ ) 

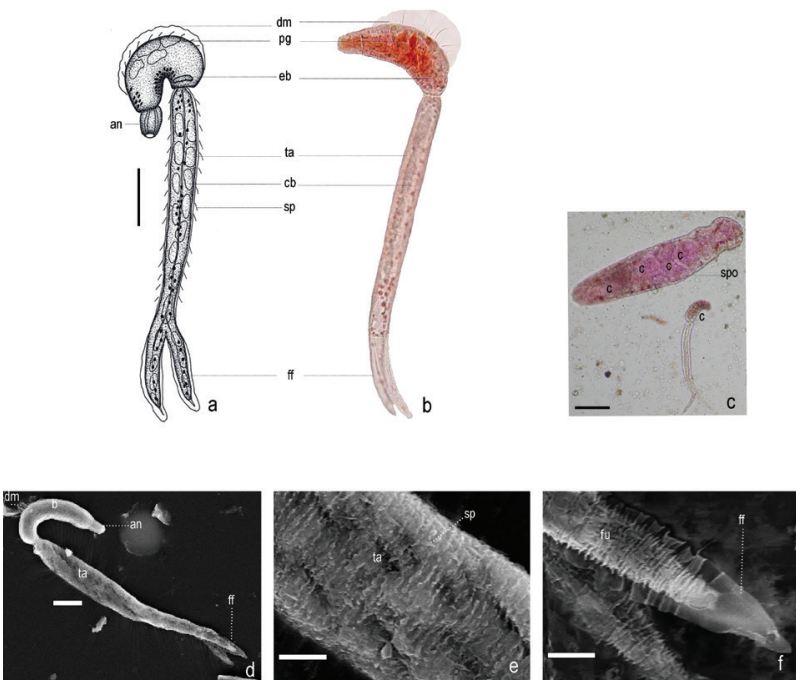

Figure 12. Image of Cardicola alseae;

a. Drawing of cercaria structure

b. Cercaria stained with Semichon's acetic carmine \& fast green

c. Sporocyst stained with $0.5 \%$ neutral red

d.- f. Images of Scanning Electronmicroscope

Abbreviations: dm - dorso-median finfold, pg - penetration gland, eb - excretory bladder, an - anterior organ, ta - tail, cb caudal body, sp - spine, fu - furca, spo : sporocyst, c - cercaria (scale $\mathrm{a}, \mathrm{b}=50 \mu \mathrm{m}, \mathrm{c}=20 \mu \mathrm{m})$.

Anterior organ:

11-15 $\mu \mathrm{m}($ av. $13 \mu \mathrm{m}) \times 14-20 \mu \mathrm{m}$ (av. $17 \mu \mathrm{m}$ )

Excretory bladder: $3-8 \mu \mathrm{m}($ av. $5 \mu \mathrm{m}) \times 10-35 \mu \mathrm{m}(\mathrm{av}$. $21 \mu \mathrm{m})$

Tail stem: $\quad 15-30 \mu \mathrm{m}($ av. $26 \mu \mathrm{m}) \times 154-197$ $\mu \mathrm{m}$ (av. $185 \mu \mathrm{m})$

Tail furcal: $\quad 7-11 \mu \mathrm{m}($ av. $9 \mu \mathrm{m}) \times 28-54 \mu \mathrm{m}(\mathrm{av}$. $51 \mu \mathrm{m})$

Dorso-median

finfold: $\quad 5-12 \mu \mathrm{m}($ av. $8 \mu \mathrm{m})$

Movement behavior: The cercaria floated on the surface of the water. The body and tail hanged rolling. It moved by folding its tail back to the body, moving forward around 6-10 seconds, and resting for 3-4 seconds. When at rest the body hangs upwards and rolls back slowly downwards with the tail stem and furcae moved upwards. It survived up to 2-3 hours in the water after emergence.

\section{Alaria mustelae Bosma, 1899 (Yamaguti, 1975)}

Alaria mustelae (Fig. 13) were found from 47 M. tuberculata which is equivalent to an infection rate of $0.15 \%$ $(47 / 32,026)$ of the total number of the collected snails (Table 2). Cercarial body has a long shape. Unpigmented eyespots lay on the midway between two suckers in lateral fields, prepharynx short, pharynx small and muscular, esophagus rather long, ceca extending a short distance
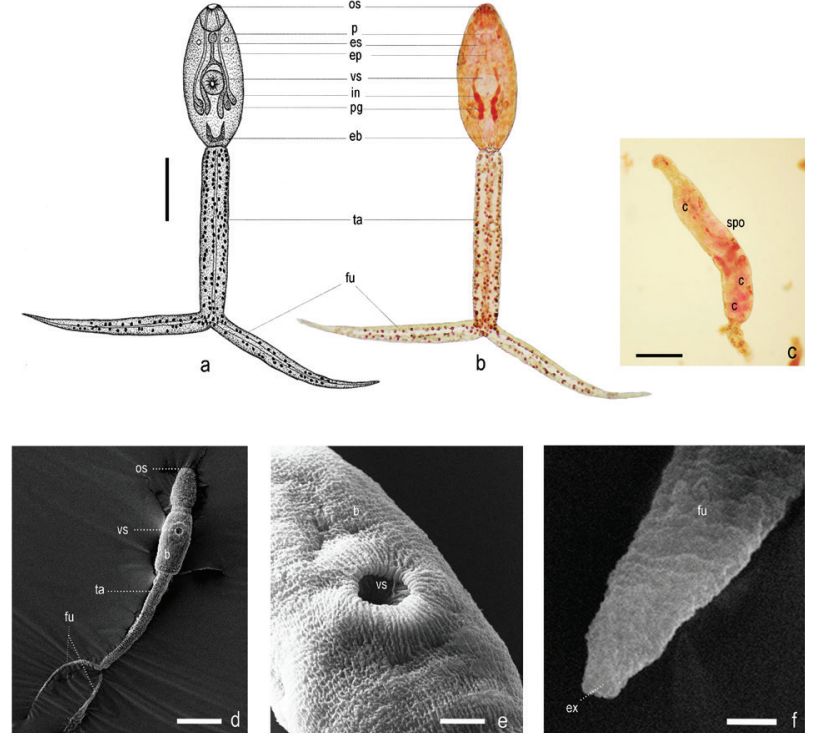

Figure 13. Image of Alaria mustelae;

a. Drawing of cercaria structure

b. Cercaria stained with $0.5 \%$ neutral red

c. Sporocyst stained with $0.5 \%$ neutral red

d.- f. Images of Scanning Electronmicroscope

Abbreviations: os - oral sucker, p - pharynx, es - eye spot, ep - esophagus, vs - ventral sucker, in - intestine, pg - penetration gland, eb - excretory bladder, ta - tail, fu - furca, spo : sporocyst, c- cercaria (scale $\mathrm{a}, \mathrm{b}=50 \mu \mathrm{m}, \mathrm{c}=10 \mu \mathrm{m}$ ).

posterior to acetabulum. The oral sucker is slightly larger than the postequatorial acetabulum. There are two pairs of penetration glands, filled with fine granules. The duct openings on each side of the mouth are in spineless circumoral area. The body is covered entirely with spines and two irregular spines around the aperture of the ventral sucker. Genital primordium is a small mass of cells anterior to the excretory vesicle. Cercaria developed within the sporocyst. The tail stem was without spines; the furcae was irregularly spinose (long hair like), with no caudal bodies. The excretory pore was found at the fork tail tip.

Size range and average size (in micrometers, calculated from 20 cercariae):

Body:

$105-154 \mu \mathrm{m}($ av. $138 \mu \mathrm{m}) \times 185-$ $280 \mu \mathrm{m}$ (av. $255 \mu \mathrm{m})$

Oral sucker: $28-40 \mu \mathrm{m}($ av. $36 \mu \mathrm{m}) \times 28-40 \mu \mathrm{m}$ (av. $36 \mu \mathrm{m}$ )

Ventral sucker: $\quad 15-30 \mu \mathrm{m}($ av. $22 \mu \mathrm{m}) \times 15-30 \mu \mathrm{m}$ (av. $22 \mu \mathrm{m}$ )

Pharynx: $\quad 10-15 \mu \mathrm{m}($ av. $13 \mu \mathrm{m}) \times 14-17 \mu \mathrm{m}$ (av. $15 \mu \mathrm{m})$

Tail stem: $\quad 48-60 \mu \mathrm{m}($ av. $55 \mu \mathrm{m}) \times 220-300$ $\mu \mathrm{m}$ (av. $260 \mu \mathrm{m})$

Tail furcae: $\quad 38-65 \mu \mathrm{m}(\mathrm{av} .60 \mu \mathrm{m}) \times 245-320$ $\mu \mathrm{m}$ (av. $280 \mu \mathrm{m})$

Movement behavior: The cercariae moved by rolling up and springing back the body to swiftly move forward 
in a quick semi-circular motion. It then rested by floating with its head on top for a long time about 20-30 seconds then moved quickly about 7-15 seconds and rested by floating again. It survived up to 2-3 hours in the water after emergence.

\section{Transversotrema laruei Velasguez, 1958 (Yamaguti, 1975)}

Transversotrema laruei (Fig. 14) were found from $89 \mathrm{M}$. tuberculata, which is equivalent to an infection rate of $0.28 \%(89 / 32,026)$ of the total number of the collected snails (Table 2). The body is in a bowl-liked shape and light brownish in color, with numerous spines like fish scales on the body surface. Many granules are observed. The genital pore of the seminal vesicle is at the anterior end of the body. There are very big round eye spots, and the ventral sucker is globular. There is a mouth on the ventral sucker. The esophagus is narrow and long, attached to the intestine. There are 1 pair of testes, with an ovary on the left side of the testes. A small excretory bladder is at the posterior end. The tail is longer than the body length with a fork tip, and one pair of appendage at the base of the tail. An adhesive pad was observed at the end of the tail. The furcal tails are spatulate. There are 4 flame cells, and an opening duct of the excretory bladder is on the furcal tail. Cercariae were produced within the rediae.

Size range and average size (in micrometers, calculated from 20 cercariae):

Body:

425-670 $\mu \mathrm{m}($ av. $574 \mu \mathrm{m}) \times 280-$ $510 \mu \mathrm{m}$ (av. $370 \mu \mathrm{m})$

Genital pore: $12-18 \mu \mathrm{m}$ (av. $15 \mu \mathrm{m}) \times 12-18 \mu \mathrm{m}$ (av. $15 \mu \mathrm{m})$

Pharynx: $25-57 \mu \mathrm{m}$ (av. $574 \mu \mathrm{m}) \times 280-510$ $\mu \mathrm{m}$ (av. $370 \mu \mathrm{m})$

Eye spot: $14-20 \mu \mathrm{m}($ av. $18 \mu \mathrm{m}) \times 14-20 \mu \mathrm{m}$ (av. $18 \mu \mathrm{m}$ )

Excretory bladder: $11-23 \mu \mathrm{m}($ av. $15 \mu \mathrm{m}) \times 11-21 \mu \mathrm{m}$ (av. $15 \mu \mathrm{m}$ )

Ventral sucker: $\quad 85-105 \mu \mathrm{m}($ av. $95 \mu \mathrm{m}) \times 85-105$ $\mu \mathrm{m}$ (av. $95 \mu \mathrm{m})$

Tail stem: $\quad 50-185 \mu \mathrm{m}($ av. $98 \mu \mathrm{m}) \times 254-570$ $\mu \mathrm{m}$ (av. $360 \mu \mathrm{m})$

Tail furcae: $\quad 45-110 \mu \mathrm{m}($ av. $66 \mu \mathrm{m}) \times 145-310$ $\mu \mathrm{m}$ (av. $204 \mu \mathrm{m})$

Appendages: $\quad 18-72 \mu \mathrm{m}($ av. $47 \mu \mathrm{m}) \times 120-250$ $\mu \mathrm{m}($ av. $160 \mu \mathrm{m})$

Movement behavior: The cercaria floated on the surface or in the middle of the water. The body sank lower than the tail. It moved very fast by turning from left to right, and moving forward in a screwing motion for about 5-8 seconds, and resting about 2-3 minutes by floating with its head and tail folded together. The furcae floated upwards, moving by wavering on the container surface.

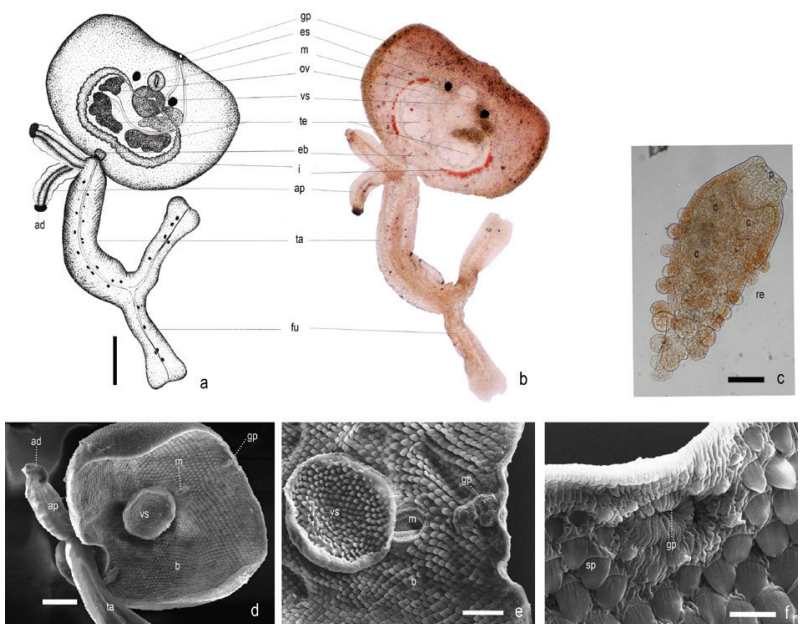

Figure 14. Image of Transversotrema laruei;

a. Drawing of cercaria structure

b. Cercaria stained with $0.5 \%$ neutral red

c. Redia stained with $0.5 \%$ neutral red

d.- f. Images of Scanning Electronmicroscope

Abbreviations: gp - genital pore, es - eye spot, $\mathrm{m}$ - mouth, ov - ovary, vs - ventral sucker, te - testes, eb - excretory bladder, $\mathrm{i}$ - intestine, ap - appendages, ta - tail, fu - furca, $\mathrm{p}$ - pharynx, re - redia, $\mathrm{c}$ - cercaria (scale $\mathrm{a}, \mathrm{b}=100 \mu \mathrm{m}, \mathrm{c}=20 \mu \mathrm{m}$ ).

\section{Apatemon gracilis Szidat, 1928 (Yamaguti, 1975)}

Apatemon gracilis (Fig. 15) were found from $55 \mathrm{M}$. tuberculata, which is equivalent to an infection rate of $0.17 \%(55 / 32,026)$ of the total number of the collected snails (Table 2). The cercarial body is oval in shape, and the prepharynx is practically absent. The anterior region has a cuticle spine. It has unpigmented eyespots. The tail stem contains about 16 caudal bodies. There are 4 pairs of penetration glands which lie between the acetabulum and genital primordia. The excretory bladder is very small. Cercariae were produced within the sporocyst.

Size range and average size (in micrometers, calculated from 20 cercariae):

Body:

41.5-90.0 $\mu \mathrm{m}($ av. $72 \mu \mathrm{m}) \times 115-$

$\begin{array}{ll}\text { Oral sucker: } & 15-25 \mu \mathrm{m} \text { (av. } 21.45 \\ & \mu \mathrm{m}(\text { av. } 23.25 \mu \mathrm{m})\end{array}$

$140 \mu \mathrm{m}($ av. $126 \mu \mathrm{m})$

Ventral sucker: $16-25 \mu \mathrm{m}($ av. $23 \mu \mathrm{m}) \times 20-30 \mu \mathrm{m}$ (av. $25 \mu \mathrm{m})$

Excretory bladder: $15-25 \mu \mathrm{m}($ av. $18 \mu \mathrm{m}) \times 15-30 \mu \mathrm{m}$ (av. $20 \mu \mathrm{m})$

Pharynx: 6-10 $\mathrm{m}$ (av. $8 \mu \mathrm{m}) \times 7-13 \mu \mathrm{m}$ (av. $9 \mu \mathrm{m})$

Tail stem: $\quad 30-45 \mu \mathrm{m}($ av. $39 \mu \mathrm{m}) \times 240-312 \mu \mathrm{m}$ (av. $286 \mu \mathrm{m})$

Tail furcae: $\quad 10-25 \mu \mathrm{m}(\mathrm{av} .18 \mu \mathrm{m}) \times 130-160 \mu \mathrm{m}$ (av. $146 \mu \mathrm{m}$ )

Movement behavior: The cercariae floated on the surface or in the water. The body sank lower than the spread- 


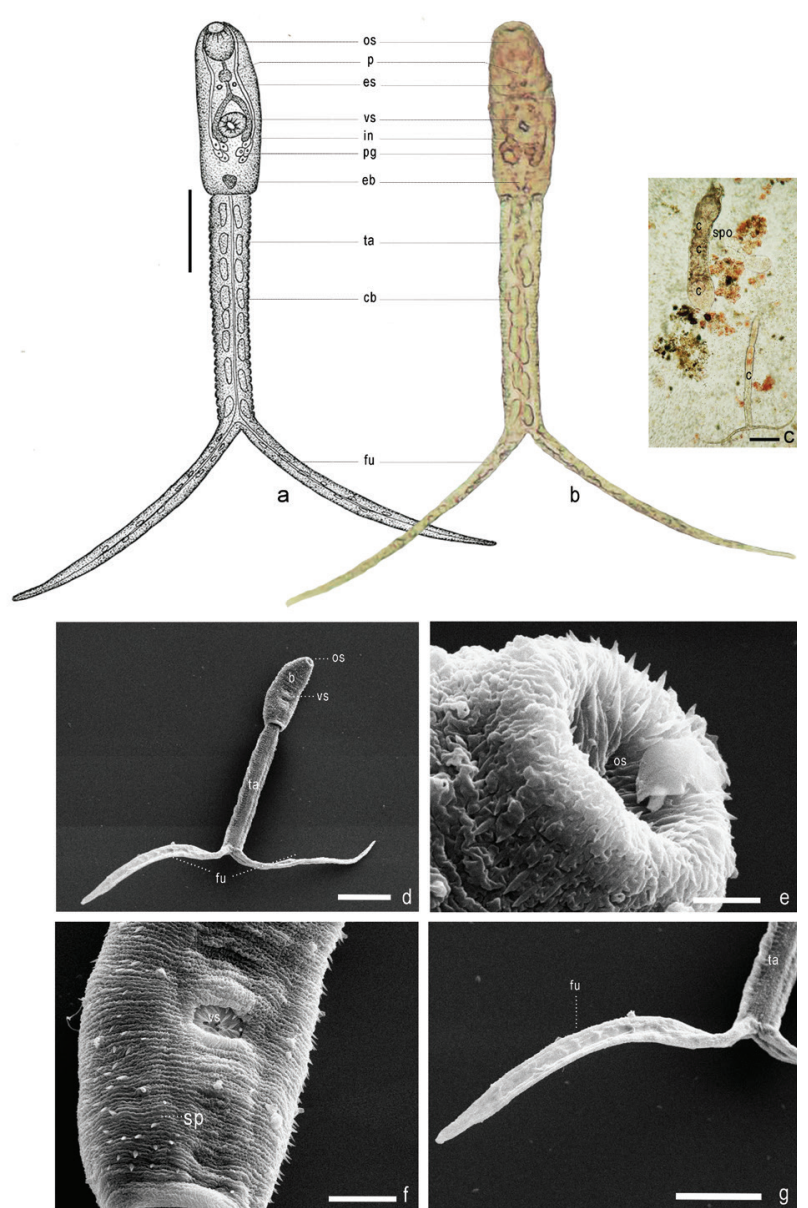

Figure 15. Image of Apatemon gracilis;

a. Drawing of cercaria structure

b. Cercaria stained with $0.5 \%$ neutral red

c. Sporocyst stained with $0.5 \%$ neutral red

d.- g. Images of Scanning Electronmicroscope

Abbreviations: os - oral sucker, p - pharynx, es - eye spot, vs - ventral sucker, in - intestine, pg - penetration gland, eb - excretory bladder, ta - tail, cb - caudal body, fu - furca, b - body, sp - spine, spo - sporocyst, c - cercaria (scale a, b $=100 \mu \mathrm{m}, \mathrm{c}$ $=20 \mu \mathrm{m})$.

ing fork tail. It moved by rolling up and springing back the body to swiftly move forward in a semi-circular motion. It then rested by floating with its head on top for about $12-15$ seconds, then moved quickly about 3-6 seconds and rested by floating again.

\section{Mesostephanus appendiculatus (Cicrea, 1916) Lutz, 1935 (Yamaguti, 1975)}

Mesostephanus appendiculatus (Fig. 16) were found from $3 \mathrm{M}$. tuberculata, which is equivalent to an infection rate of $0.009 \%(3 / 32,026)$ of the total number of the collected snails (Table 2). The cercarial body is oval in shape and spinose. There are many spines on the surface and the oral sucker. Coarse granules and cytogenous gland are scattered inside the body. The pharynx is small and round. The prepharynx and esophagous are almost as long as the pharynx.
A large intestine, composed of two cecae, is terminated near a small excretory bladder. A ventral sucker vestigial is found to be in small groups. The tail is forked and longer than the body and the tail surface is covered with many spines. The tail stem is longer than the furca. The tail tubule opens at the tip of each tail furca in which no flame cell is found. Cercariae were produced within the sporocyst.

Size range and average size (in micrometers, calculated from 20 cercariae):

Body:

92-120 $\mu \mathrm{m}($ av. $109 \mu \mathrm{m}) \times 160-250$ $\mu \mathrm{m}$ (av. $225 \mu \mathrm{m})$

Oral sucker: $\quad 20-45 \mu \mathrm{m}($ av. $35 \mu \mathrm{m}) \times 35-48 \mu \mathrm{m}$ (av. $39 \mu \mathrm{m})$

Ventral sucker: $\quad 15-24 \mu \mathrm{m}($ av. $20 \mu \mathrm{m}) \times 15-24 \mu \mathrm{m}$ (av. $20 \mu \mathrm{m}$ )

Excretory bladder: $20-40 \mu \mathrm{m}$ (av. $35 \mu \mathrm{m}) \times 20-40 \mu \mathrm{m}$ (av. $35 \mu \mathrm{m})$

Pharynx: $\quad 8-10 \mu \mathrm{m}($ av. $9 \mu \mathrm{m}) \times 11-18 \mu \mathrm{m}($ av . $15 \mu \mathrm{m})$
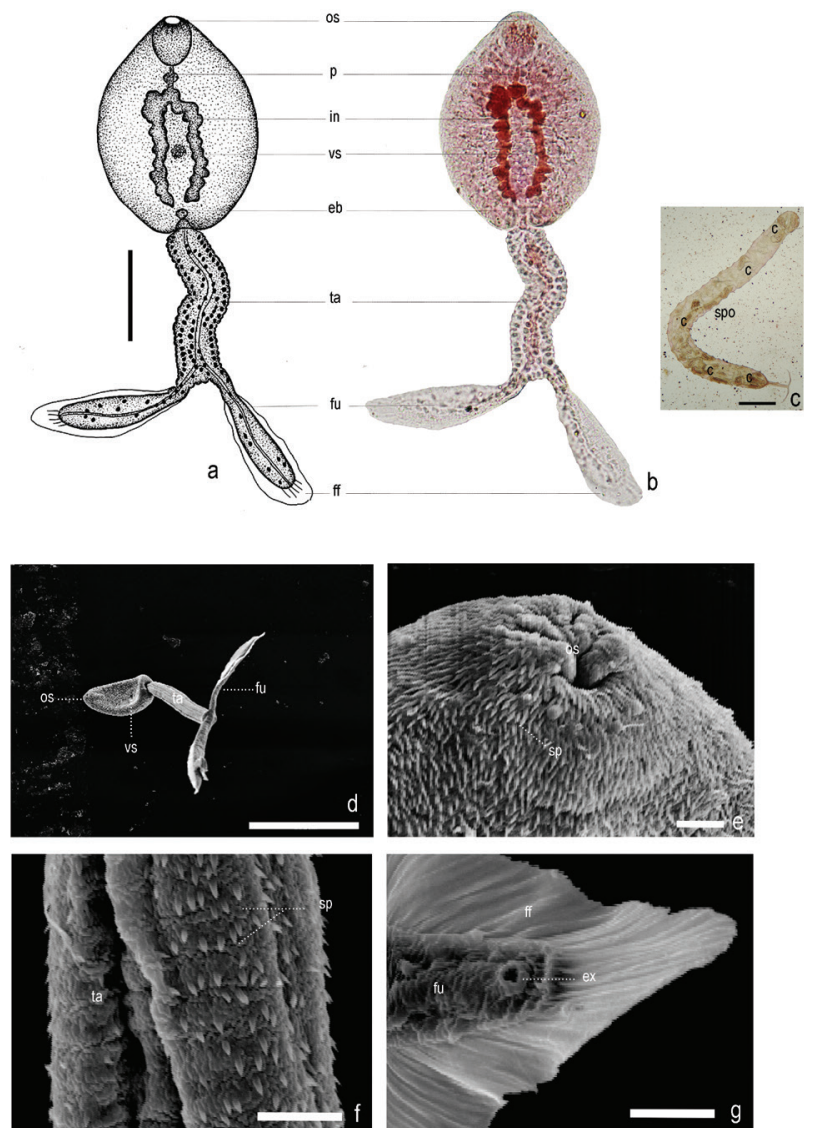

Figure 16. Image of Mesostephanus appendiculatus;

a. Drawing of cercaria structure

b. Cercaria stained with $0.5 \%$ neutral red

c. Sporocyst stained with $0.5 \%$ neutral red

d.- g. Images of Scanning Electronmicroscope

Abbreviations: os - oral sucker, $\mathrm{p}$ - pharynx, in - intestine, vs - ventral sucker, eb - excretory bladder, ta - tail, fu - furca, ff furcal finfold $\mathrm{b}$ - body, sp - spine, ex - excretory pore, spo - sporocyst, $\mathrm{c}$ - cercaria (scale a, $\mathrm{b}=100 \mu \mathrm{m}, \mathrm{c}=20 \mu \mathrm{m}$ ). 
Tail stem:

$25-40 \mu \mathrm{m}($ av. $35 \mu \mathrm{m}) \times 425-525 \mu \mathrm{m}$ (av. $495 \mu \mathrm{m})$

Tail furcae:

15-25 $\mu \mathrm{m}($ av. $20 \mu \mathrm{m}) \times 160-170$ $\mu \mathrm{m}($ av. $165 \mu \mathrm{m})$

Movement behavior: The cercaria floated on the surface or in the water. The body sank lower than the spreading fork tail. It moved by rolling up and springing back the body to swiftly move forward in a semi-circular motion for about 2-4 seconds. It then rested by floating with its head on top of the tail and slowly rotated its body to the bottom while lifting up its spreading fork tail. It rested for about 20-30 seconds and survived up to 2-3 hours in the water.

\section{Type 6. Echinostome cercariae}

15. Echinochasmus pelecani Johnston \& Simpson, 1944 (Yamaguti, 1975)

Echinochasmus pelecani (Fig. 17) were found from 19 $M$. tuberculata which is equivalent to an infection rate of $0.06 \%(19 / 32,026)$ of the total number of the collected snails (Table 2). Cercarial body is elongate, white in
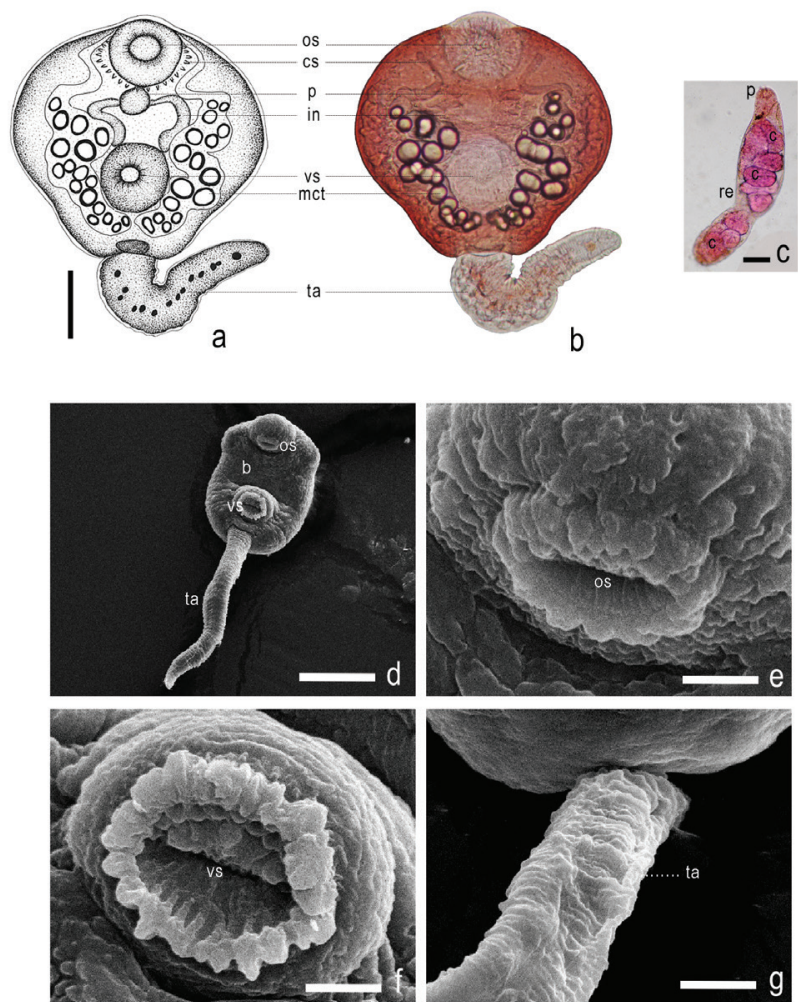

Figure 17. Image of Echinochasmus pelecani;
a. Drawing of cercaria structure
b. Cercaria stained with $0.5 \%$ neutral red
c. Redia stained with $0.5 \%$ neutral red
d.- g. Images of Scanning Electronmicroscope

Abbreviations: os - oral sucker, cs - collar spine, p - pharynx, in - intestine, vs - ventral sucker, mct - main collecting tube, ta - tail, re - redia, $\mathrm{c}$ - cercaria (scale a, $\mathrm{b}=50 \mu \mathrm{m}, \mathrm{c}=10 \mu \mathrm{m}$ ).

color, no eye spot, oral sucker with 3 opening of duct ( 1 median, 2 submediant), cystogenous cell containing rhabdites. Collar spine is not appearance. Esophagus was between pharynx and ventral sucker, ceca reaching to bladder, two main excretory tubes meet together before entering bladder, Genital primordia are two mass behind ventral sucker, no penetration gland, flame cell pattern not determine. Tail is the same length as body, flexible, vacuole appearance along the tail. Cercariae were produced within rediae.

Size range and average size (in micrometers, calculated from 20 cercariae):

Body:

Oral sucker:

Ventral sucker:

Pharynx:

Excretory bladder: $21-35 \mu \mathrm{m}($ av. $33 \mu \mathrm{m}) \times 23-36 \mu \mathrm{m}$ (av. $33 \mu \mathrm{m}$ )

Tail: 23-38 $\mu \mathrm{m}$ (av. $35 \mu \mathrm{m}) \times 95-130 \mu \mathrm{m}$ (av. $115 \mu \mathrm{m}$ )

Movement behavior: The cercaria floated on the surface or in the water. It moved very fast by rolling up and springing back about 5-10 seconds. It survived up to 3-4 hours in the water after emergence.

\section{Type 7. Amphistome cercariae}

\section{Gastrothylax crumenifer (Creplin, 1847) Otto, 1896 (Yamaguti, 1975)}

Gastrothylax crumenifer (Fig. 18) were found from 8 $M$. tuberculata which is equivalent to an infection rate of $0.02 \%(8 / 32,026)$ of the total number of the collected snails (Table 2). Cercariae were liberated from the rediae. The body shape is ovate and large. The eye spots have conical lens with yellow pigment through the body with a smooth surface. The ceca ended 0.14-0.17 mm away from the posterior end of the body, with symmetrical testes at the level of the ceca end. The oral sucker is equal to the ventral sucker. The tail inserted to the posterior end of the body. There are various sizes of vacuole through the tail.

Size range and average size (in micrometers, calculated from 20 cercariae):

Body:

Oral sucker:

Ventral sucker:

Pharynx:
$190-250 \mu \mathrm{m}($ av. $220 \mu \mathrm{m}) \times 350-$ $415 \mu \mathrm{m}($ av. $370 \mu \mathrm{m})$

$45-65 \mu \mathrm{m}($ av. $52 \mu \mathrm{m}) \times 45-65 \mu \mathrm{m}$ (av. $52 \mu \mathrm{m})$

$48-68 \mu \mathrm{m}($ av. $55 \mu \mathrm{m}) \times 48-68 \mu \mathrm{m}$ (av. $55 \mu \mathrm{m})$

8-10 $\mu \mathrm{m}$ (av. $10 \mu \mathrm{m}) \times 8-12 \mu \mathrm{m}$ (av. $11 \mu \mathrm{m})$ 

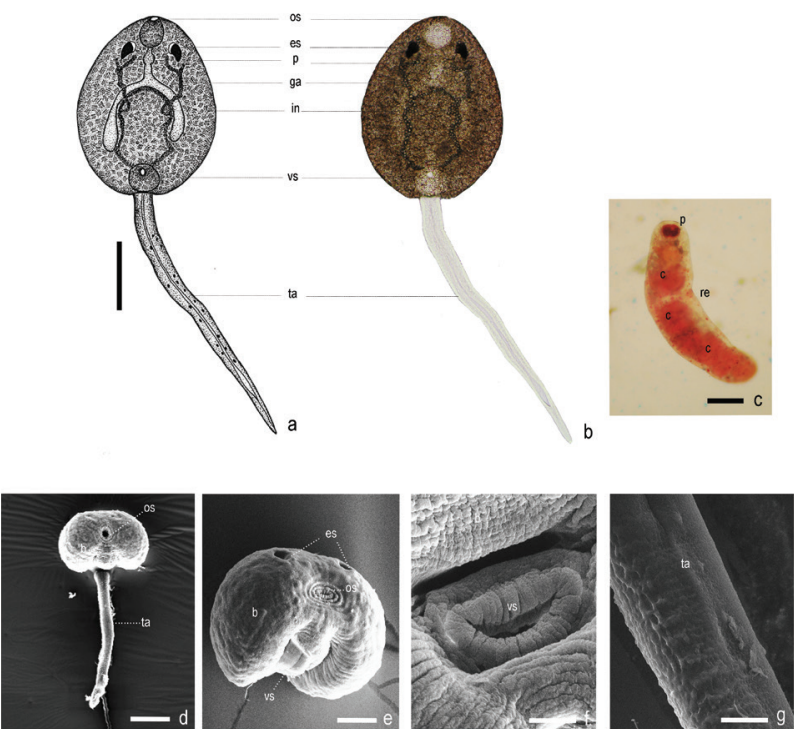

Figure 18. Image of Gastrothylax crumenifer;

a. Drawing of cercaria structure

b. Cercaria stained with $0.5 \%$ neutral red

c. Redia stained with $0.5 \%$ neutral red

d.- g. Images of Scanning Electronmicroscope

Abbreviations: os - oral sucker, p - pharynx, es - eye spot, vs - ventral sucker, ga - genital atrium, in - intestine, vs - ventral sucker, ta - tail, re - redia, c - cercaria (scale a, b $=100 \mu \mathrm{m}, \mathrm{c}$ $=10 \mu \mathrm{m})$.

Esophagous:

80-140 $\mu \mathrm{m}$ (av. $125 \mu \mathrm{m}) \times 80-140$ $\mu \mathrm{m}(\mathrm{av} .125 \mu \mathrm{m})$

Tail: 65-95 $\mu \mathrm{m}($ av. $82 \mu \mathrm{m}) \times 328-450$ $\mu \mathrm{m}$ (av. $410 \mu \mathrm{m})$

Movement behavior: The cercaria floated on the surface or in the water. It moved by wavering on the surface of the water for around 8-10 seconds, and then rolling up and springing back for about 5-10 seconds. It survived up to 3-4 hours in the water after emergence. The cercariae were photo-sensitive. They shrank rapidly in changing light conditions.

\section{Type 8. Renicolid cercariae}

\section{Cercaria caribbea LXVIII (Cable, 1963) (Yamagu- ti, 1975)}

Cercaria caribbea LXVIII (Fig. 19) were found from 45 M. tuberculata which is equivalent to an infection rate of $0.14 \%(45 / 32,026)$ of the total number of the collected snails (Table 2). Cercariae developed in the sporocyst. Its body is flat with yellow pigment and numerous minute spines on the surface of the body, with no eye spot, a small sucker of 28-36 $\mu \mathrm{m}$, a short prepharynx, a pharynx of $12-14 \mu \mathrm{m}$, plenty of small cephalic glands in the middle of the body, a short excretory vesicle split into two since the upper of the acetabulum. The tail is straight, and is longer than the length of the body, with no lateral finfold, and no flame cells.
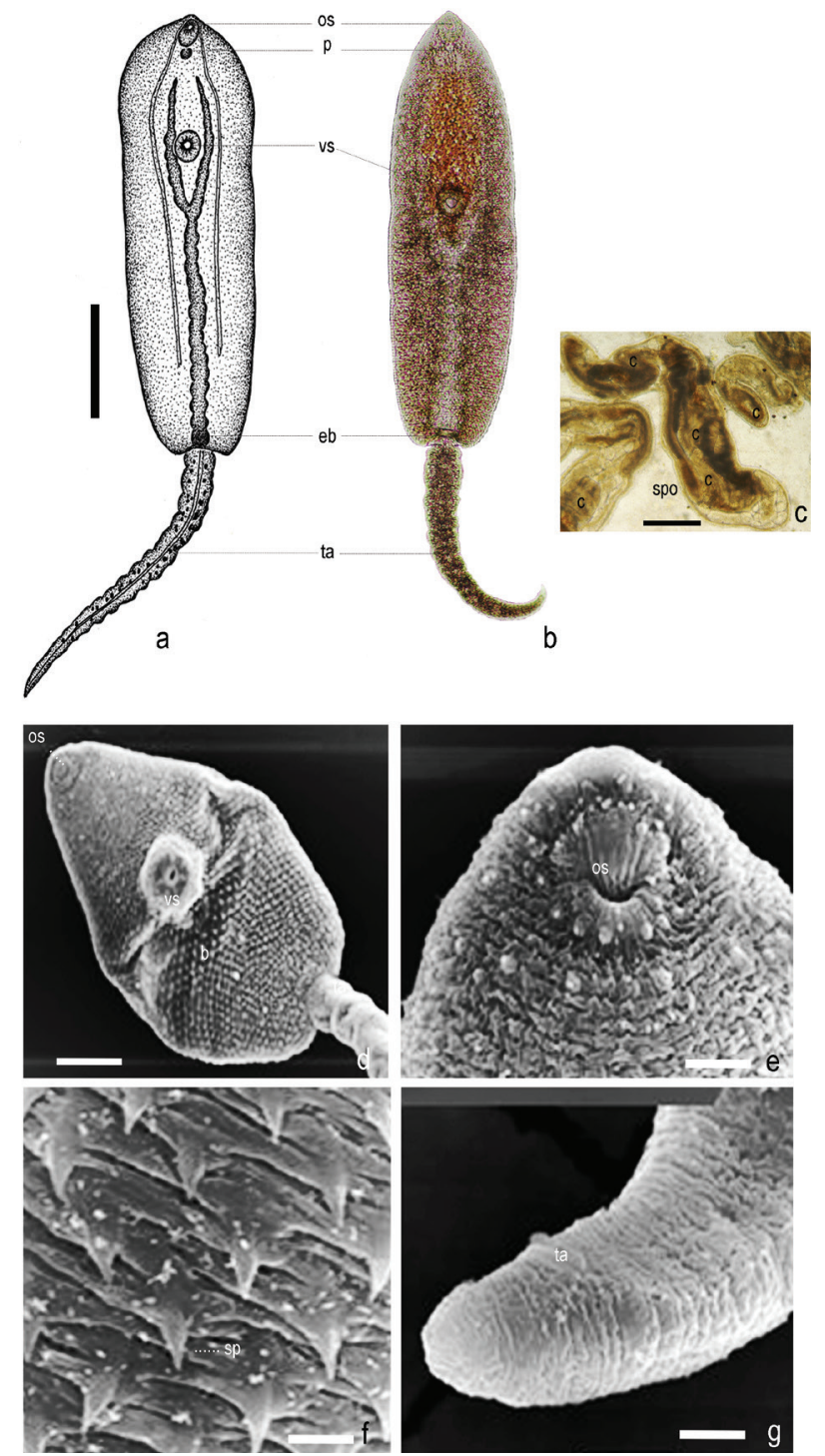

Figure 19. Image of Cercaria caribbea LXVIII;

a. Drawing of cercaria structure

b. Cercaria stained with $0.5 \%$ neutral red

c. Sporocyst stained with $0.5 \%$ neutral red

d.- g. Images of Scanning Electronmicroscope

Abbreviations: os - oral sucker, p - pharynx, vs - ventral sucker eb - excretory bladder, ta - tail, spo - sporocyst (scale a, $b=100$ $\mu \mathrm{m}, \mathrm{c}=10 \mu \mathrm{m})$.

Size range and average size (in micrometers, calculated from 20 cercariae):

Body:

$128-140 \mu \mathrm{m}($ av. $129 \mu \mathrm{m}) \times 390-$ $435 \mu \mathrm{m}$ (av. $420 \mu \mathrm{m})$

Oral sucker: $\quad 28-33 \mu \mathrm{m}($ av. $26 \mu \mathrm{m}) \times 28-33 \mu \mathrm{m}$ (av. $26 \mu \mathrm{m})$

Ventral sucker: $\quad 29-36 \mu \mathrm{m}($ av. $30 \mu \mathrm{m}) \times 29-36 \mu \mathrm{m}$ (av. $30 \mu \mathrm{m})$

Pharynx: $\quad 8-14 \mu \mathrm{m}($ av. $12 \mu \mathrm{m}) \times 10-14 \mu \mathrm{m}$ (av. $12 \mu \mathrm{m})$

Excretory bladder: $\quad 15-20 \mu \mathrm{m}($ av. $17 \mu \mathrm{m}) \times 15-20 \mu \mathrm{m}$ (av. $17 \mu \mathrm{m}$ ) 
Tail:

$$
\begin{aligned}
& \text { 34-38 } \mu \mathrm{m}(\text { av. } 35 \mu \mathrm{m}) \times 395-480 \\
& \mu \mathrm{m}(\text { av. } 450 \mu \mathrm{m})
\end{aligned}
$$

Movement behavior: The cercaria moved slowly on the bottom of the container, and swam continuously. It survived up to 2-3 hours in the water after emergence.

\section{Type 9. Cotylomicrocercous cercariae}

\section{Podocotyle (Podocotyle) lepomis Dobrovolny, 1939 (Yamaguti, 1975)}

Podocotyle lepomis (Fig. 20) were found from 3 M. tuberculata which is equivalent to an infection rate of $0.009 \%$ $(3 / 32,026)$ of the total number of the collected snails (Table 2). Cercariae developed in the sporocyst. The body is cylindrical in shape, clear white in color, with no sensory hair, spine, 6 papillae on the head, and rough granules present on the body. The stylet is present in oral sucker. There were 2 rows of sensory papillae around the oral sucker, with a long prepharynx. Pharynx is round. The ceca extended to the posterior end, with 5 pairs of penetration glands. 2 of them were not stained with $0.5 \%$ neutral red while 3 of them were stained with $0.5 \%$ neutral red. Excretory vesicle had a thick wall and open dorsally at the tail, the flame cell formula is $2[(2+2)+(2+2)]$. The tail is short, only half the length of the body, cup-shaped, with an adhesive gland present at the end of the tail for attaching.

Size range and average size (in micrometers, calculated from 20 cercariae):

Body: $\quad 65-93 \mu \mathrm{m}($ av. $73 \mu \mathrm{m}) \times 103-145$ $\mu \mathrm{m}($ av. $123 \mu \mathrm{m})$
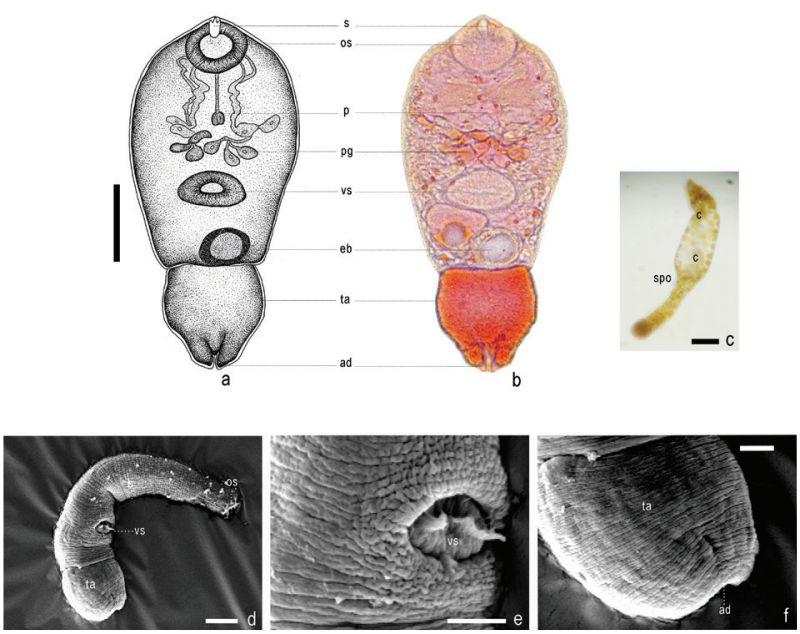

Figure 20. Image of Podocotyle (Podocotyle) lepomis;

a. Drawing of cercaria structure

b. Cercaria stained with $0.5 \%$ neutral red

c. Sporocyst stained with $0.5 \%$ neutral red

d.- f. Images of Scanning Electronmicroscope

Abbreviations: s - stylet, os - oral sucker, $\mathrm{p}$ - pharynx, penetration gland, vs - ventral sucker, eb - excretory bladder, ta - tail, ad - adhesive organ, spo - sporocyst, $\mathrm{c}$ - cercaria (scale a, b = $100 \mu \mathrm{m}, \mathrm{c}=10 \mu \mathrm{m})$.
Stylet:

Oral sucker:

Ventral sucker:

Pharynx:

Excretory bladder:

Tail:

4-6 $\mu \mathrm{m}($ av. $5 \mu \mathrm{m}) \times 6-10 \mu \mathrm{m}(\mathrm{av} .8$ $\mu \mathrm{m})$

$30-38 \mu \mathrm{m}($ av. $32 \mu \mathrm{m}) \times 25-38 \mu \mathrm{m}$ (av. $30 \mu \mathrm{m}$ )

$28-40 \mu \mathrm{m}($ av. $35 \mu \mathrm{m}) \times 28-40 \mu \mathrm{m}$ (av. $35 \mu \mathrm{m}$ )

13-18 $\mu \mathrm{m}$ (av. $14 \mu \mathrm{m}) \times 10-13 \mu \mathrm{m}$ (av. $11 \mu \mathrm{m}$ )

$20-30 \mu \mathrm{m}($ av. $26 \mu \mathrm{m}) \times 18-25 \mu \mathrm{m}$ (av. $21 \mu \mathrm{m}$ )

43-65 $\mu \mathrm{m}($ av. $52 \mu \mathrm{m}) \times 50-75 \mu \mathrm{m}$ (av. $61 \mu \mathrm{m}$ )

Movement behavior: The cercaria floated with the ventral upside. It moved by floating with its head and tail folded together, and then sprang up. Normally it creeps on the surface of the container using the oral and ventral sucker. The cercaria floated for about 15-20 seconds, and rested for about 10-15 seconds. It survived up to 2-3 hours in the water.

\section{Discussion}

Melanoides tuberculata Müller, 1774 is a benthic freshwater thiarid native to Africa and Asia. Its original native range seemed uncertain but nonetheless wide, including parts of Africa, the Mediterranean, Asia and the Pacific Islands (Pace 1973, Clench 1969). Glaubrecht (1996) hypothesized that its origin lies rather in Asia than Africa, given its natural occurrences and the history of Thiaridae; see also Glaubrecht et al. (2009) and Glaubrecht (2011). It had also become established in several other countries. (Facon et al. 2004, Derraik 2008). Aquarium plants exchange by humans caused the brake down of natural dispersal barriers to these snails (Kolar and Lodge 2001). Moreover, Melanoides had also adapted well to new environments. For example, on Martinique Island M. tuberculata was surveyed in 1979 and 2003. While there were only two morphs found in 1979, in 2003 there were nine morphs. Population dynamics and distribution of $M$. tuberculata were studied in many areas, where they dominated the streams, ponds, and lakes (e.g. Supian and Ikhwanuddin 2002, Eldblom and Kristensen 2003, Facon et al. 2003).

Melanoides tuberculata was found to be intermediate host for a number of trematode parasites (e.g. Pinto and De Melo 2011, Ukong et al. 2007). As a consequence, the introduction of $M$. tuberculata leads to new parasitic cycles in humans in the invaded area. However, there are also reports that show the efficacy of $M$. tuberculata as a biocontrol agent against the schistosome (blood fluke) vector snails Biomphalaria glabrata (Pointier and Jourdane 2000).

In Thailand, Brandt (1974) reported that thiarid snails were found in lakes, ponds, marshes, canals, streams, rivers, and other sources of river such as waterfalls. In the present work, we did a smaller scale of investigation than Brandt's. More than thirty thousand of M. tubercu- 
lata snails were collected from rice paddies, drainages, ponds, canals, water reservoirs, marshes, streams, waterfalls, and rivers in Thailand. The classification of $M$. tuberculata was performed as reported by Brandt (1974). It was quite clear to distinguish $M$. tuberculata from other thiarids, although among these snails there were quite different shell morphologies in terms of ribs, color, pigmentation and even size. Although, based on the shell, the destinction of discrete several morphs was possible (see e.g. Pointier 1989, 1993, Samadi et al. 1999), all of these morphs were still considered to be conspecific within $M$. tuberculata. It is hoped that molecular techniques will help us to eventually solve this question of intraspecific versus intraspecific variation.

In this study, the recovery of adult trematode stages are not completely recorded, but the morphological distinction of cercariae are quite clear from the unstained, stained and electronmicroscopic images, allowing to classify the eighteen species of cercariae from this thiarid snail species into nine types of cercarial morphology, as compiled in the Result section.

In the present study, we also found human trematodes, viz. Haplorchis taichui, Haplorchis pumilio, Centrocestus formosanus and Cercaria caribbea LXVIII. Especially the $H$. pumilio human minute intestinal fluke $(371 / 6,019$ $=6.16 \%$ ) showed a high level of prevalence in Thailand. As they complete their complex life cycle not only in humans but also in other vertebrates, it is difficult to control their infection. H. taichui is another important minute intestinal fluke. Three cases of humans were reported with mucosal ulceration, mucosal and submucosal haemorrhages, fusion and shortening villi, chronic inflammation and fibrosis of submucosa; in addition, there was a report of the pathology in the small intestine of patients caused by H. taichui (Sukontason et al. 2005). In earlier reports, Haplorchis spp. were found to be of high prevalence of infection in the north of Thailand (Chontananarth and Wongsawad 2010). In the present report, we now found Haplorchis infections in every region of Thailand.

The minute intestinal flukes were reported not only in Asia but also in South America. The life cycle of $H$. pumilio was studied from redia to adult under natural and experimental conditions in the digestive gland of Melanoides tuberculata, collected from Agasanta, Venezuela (Diaz et al. 2008). It seems that $M$. tuberculata was one of the important intermediate host snails of humans and animal trematodes in the world.

For animal parasites, the cercariae with the highest prevalence were L. bicolor $(2,373 / 6,019=39.43 \%)$, an amphibian trematode. However, the other animal parasites were also very important for public health. For example, the eye fluke Philophthalmus spp. are parasites of birds, using a snail intermediate host and birds as the definitive host. However, they also occurred in human and other animals. Human infection by these eye flukes occur via direct contact with the eye by cercariae in the water or by ingestion of cercariae in contaminated water (Alicata 1962, Waikagul et al. 2006, Derraik 2008).

One of heterophyid trematodes, Stictodora tridactyla was reported that they occasionally infested brackish water and marine snails, while metacercariae encyst in fish, with the definitive hosts being birds and mammals including humans (Chai et al. 1988, Abdul-Salam et al. 2000). S. tridactyla also infected M. tuberculata with other cercariae as well. The infection rate of $S$. tridactyla is $6.81 \%(2,182 / 6,019)$. In our previous study, we found $S$. tridactyla in M. tuberculata and even more in M. jugicostis (Ukong et al. 2007).

The highest infection rate of parasite is $7.41 \%$ $(2,373 / 6,019)$ with Loxogenoides bicolor being the most common parasite found in the present study. This parasite is one of the Xiphidiocercariae, being produced by trematodes from the superfamily Plagiorchioidea (Schell 1962, Malek and Cheng 1974). They were found in other thiarid snails, such as Thiara (i.e. Plotia) scraba at Erawan Waterfall, Kanchanaburi, Thailand (Ukong et al. 2007).

Interestingly, it can cause double infection or even triple infection in $M$. tuberculata together with other trematodes. We found a total of 326 double infections and 13 triple infections in M. tuberculata (Table 4). S. tridactyla and L. bicolor were found to be common in double infections, while S. tridactyla, L. bicolor and C. alseae were commonly found triple infections. C. alseae is a blood-dwelling trematode.

Table 4. Double trematode infections and triple trematode infections of collected Melanoides tuberculata.

\begin{tabular}{l|l|c}
\hline Infections & \multicolumn{1}{c}{ Type } & No. of infected snails \\
\hline \multirow{5}{*}{ Double } & Loxogenoides bicolor + Stictodora tridactyla & 135 \\
\cline { 2 - 3 } & Cardicola alseae + Stictodora tridactyla & 162 \\
\cline { 2 - 3 } & Apatemon gracilis + Stictodora tridactyla & 3 \\
\cline { 2 - 3 } & Loxogenoides bicolor + Cercaria caribbea LXVIII & 3 \\
\cline { 2 - 3 } & Loxogenoides bicolor + Philophthalmus sp. & 7 \\
\cline { 2 - 3 } & Loxogenoides bicolor + Haplorchis pumilio & 10 \\
\cline { 2 - 3 } & Alaria mustelae + Haplorchis pumilio & 1 \\
\cline { 2 - 3 } & Haplorchis taichui + Alaria mustelae & 1 \\
\cline { 2 - 3 } & Haplorchis taichui + Loxogenoides bicolor & 2 \\
\cline { 2 - 3 } & Haplorchis taichui + Acanthatrium hitaense & 1 \\
\hline Triple & Loxogenoides bicolor + Stictodora tridactyla + Podocotyle lepomis & 12 \\
\cline { 2 - 3 } & Loxogenoides bicolor + Stictodora tridactyla + Cardicola alseae & 339 \\
\hline
\end{tabular}


This parasite is furcocercous cercariae. It is produced by trematodes from the family Sanguinicolidae. Found in freshwater fishes they were reported to have sporocysts that developed in the visceral mass of the snail Oxytrema silicula from Alsae River, Oregon, USA (Meade 1967). In Thailand, $C$. alseae was also found in the thiarid snail Tarebia granifera at Erawan Waterfall (Ukong et al. 2007). The others furcocercous cercariae Alaria mustelae, Transversotrema laruei, Apatemon gracilis, and Mesostephanus appendiculatus, can also be found in $M$. tuberculata. These parasites, such as Transversotrema laruei and Apatemon gracilis, were found with metacercariae in brackish and freshwater fishes. The adult stages of these flukes inhibit the small intestine of their bird hosts (Smith and Hickman 1983).

Although the counts per unit of time method (Olivier and Schneiderman 1956) used to measure the density of the snail population in the marked areas does not represent the total population, our observations were performed all year round. That way we were able to document seasonal variation in the intensity of parasitism in M. tuberculata. Thus, we consider our data of cercariae infection rates from this research to be reliable. Since most of the above cited intestinal flukes affect humans and animals alike (Derraik 2008), in conclusion we can state that the thiarid snail Melanoides tuberculata can doubtlessly be considered as of considerable medical significance in Thailand and elsewhere.

\section{Acknowledgements}

This research was supported by the Research and Development Institute, Silpakorn University, Thailand. We also thank Department of Biology, and Faculty of Science, Silpakorn University. We would like to especially thank Assist. Prof. Dr. Chalermsri Chantasingh and PD. Dr. Matthias Glaubrecht for valuable suggestions and editing the manuscript.

\section{References}

Abdul-Salam J, Sreelatha BS, Ashkanani H (2000) Surface ultrastructure of Stictodora tridactyla (Trematoda: Heterophyidae) from $\mathrm{Ku}$ wait Bay. Parasitology International 49: 1-7.

Abdul-Salam J, Sreelatha BS, Ashkanani H (2004) The eye fluke Philophthalmus hegeneri (Digenea: Philophthalmidae) in Kuwait Bay. Kuwait Journal of Science \& Engineering 31: 119-133.

Alicata JE (1962) Life cycle and developmental stages of Philophthalmus gralli in the intermediate and final hosts. Journal of Parasitology 48: 47-54.

Brandt AM (1974) The non-marine aquatic mallusca of Thailand. Archiv für Molluskenkunde 105 (1/4): 1-423.

Chai JY, Hong SJ, Lee SH, Seo BS (1988) Stictodora sp. (Trematoda: Heterophyidae) recoverred from a mam in Korea. The Korean Journal of Parasitology 26: 127-132.
Chai JY, Murrell KD, Lymbery AJ (2005) Fish-borne parasitic zoonoses: status and issues. International Journal of Parasitology 35: 1233-1254.

Chontananarth C, Wongsawad C (2010) Prevalence of Haplorchis taichui in field collected snails: A molecular approach. The Korean Journal of Parasitology 48 (4): 343-346.

Chuboon S, Wongsawad C, Ruamsuk A, Nithikathkul C (2005) Survival of Haplorchis taichui metacercariae in Lab-pla, Thai traditional food preparation. The Southeast Asian Journal of Tropical Medicine and Public Health 36 (suppl 4): 110-111.

Clench WJ (1969) Melanoides tuberculata (Müller) in Florida. Nautilus 83: 72 .

Davis GM (1971) Systematic studies of Brotia costula episcopalis, first intermediate host of Paragonimus westermani in Malaysia. Proceedings of the Academy of Natural Sciences of Philadelphia 123: 53-66.

Dechruksa W, Krailas D, Ukong S, Inkapatanakul W, Koonchornboon T (2007) Trematode infections of the freshwater snail family Thiaridae in the Khek river, Thailand. The Southeast Asian Journal of Tropical Medicine and Public Health 38 (6): 1016-1028.

Dechruksa W, Krailas D, Glaubrecht M (2013) Evaluating the status and identity of “Melania” jugicostis Hanley \& Theobald, 1876-an enigmatic thiarid gastropod in Thailand (Caenogastropoda, Cerithioidea). Zoosystematics and Evolution 89 (2): 293-309.

Derraik JGB (2008) The potential significance to human health associated with the establishment of the snail Melanoides tuberculata in New Zealand. The New Zealand Medical Journal 121: 25-32.

Diaz MT, Hernandez LE, Bashirullah AK (2008) Studies on the life cycle of Haplorchis pumilio (Looss, 1896) (Trematoda: Heterophidae) in Venezuela. Revista Científica FCV-LUZ 18(1): 35-42.

Eldblom C, Kristensen TK (2003) A revision of the genus Melanoides (Gastropoda: Thiaridae) in Lake Malawi. African Zoology 38(2): 357-369.

Facon B, Machline E, Pointier JP, David P (2004) Variation in desiccation tolerance in freshwater snails and its consequences for invasion ability. Biological Invasions 6: 283-293.

Facon B, Pointier JP, Glaubrecht M, Poux C, Jarne P, David P (2003) A molecular phylogeography approach to biological invasion of the new world by parthenogenetic thiarid snails. Molecular Ecology 12: 3027-3039.

Glaubrecht M (1996) Evolutionsökologie und Systematik am Beispiel von Süß- und Brackwasserschnecken (Mollusca: Caenogastropoda: Cerithioidea): Ontogenese-Strategien, paläontologische Befunde und Historische Zoogeographie. Backhuys Publishers. Leiden. [in German with extended English abstract]

Glaubrecht M (1999) Systematics and the evolution of viviparity in tropical freshwater gastropods (Cerithioidea: Thiaridae sensu lato) - an overview. Courier Forschungs-Institut Senckenberg 215: 91-96.

Glaubrecht M (2000) A look back in time: Toward an historical biogeography as synthesis of systematic and geologic patterns outlined with limnic gastropods. Zoology 102: 127-147.

Glaubrecht M (2011) Towards solving Darwin's "mystery": Speciation and radiation in lacustrine and riverine freshwater gastropods. The American Malacological Society 29: 187-216.

Glaubrecht M, Brinkmann N, Pöppe J (2009) Diversity and disparity 'down under': Systematics, biogeography and reproductive modes of the 'marsupial' freshwater Thiaridae (Caenogastropoda, Cerithioidea) in Australia. Zoosystematics and Evolution 85(2): 199-275. 
Hotez PJ, Molyneux DH, Fenwick A, Kumaresan J, Ehrlich Sachs S, Sachs JD, Savioli L (2007) Control of neglected tropical diseases. New England Journal of Medicine 357: 1018-1027.

Hotez PJ, Fenwick A, Savioli L, Molyneux DH (2009) Rescuing the "bottom billion" through neglected tropical disease control. The Lancet 373: 1570-1574.

Ito J (1980) Studies on cercariae in Japan. Shizuoka University. Oya, Surugaku, Shizuoka.

Kolar CS, Lodge DM (2001) Progress in invasion biology: predicting invaders. Trends in Ecology and Evolution 19: 199-204.

Krailas D, Dechruksa W, Ukong S, Janecharut T (2003) Cercarial infection in Paludomus petrosus, Freshwater snail in Pa La-U Waterfall. The Southeast Asian Journal of Tropical Medicine and Public Health 34: 286-290.

Krailas D, Chotesaengsri S, Pattaradussadee N, Notesiri N, Dechruksa W (2008) Bucephalid (Gasterostome) cercariae obtained from freshwater clams in Thailand. The Journal of Tropical Medicine and Parasitology 31: 70-76.

Lydeard C, Holznagel WE, Glaubrecht M, Ponder WF (2002) Molecular phylogeny of a circum-global, diverse gastropod superfamily (Cerithioidea: Mollusca: Caenogastropoda): pushing the deepest phylogenetic limits of mitochondrial LSU rDNA sequences. Molecular Phylogenetics and Evolution 22 (3): 399-406.

Madsen H, Frandsen F (1989) The spread of fresh water snails including those of medical and veterinary importance. Acta Tropica 46: 139-149.

Malek EA, Cheng TC (1974) Medical and Economic malacology. Academic Press, New York

Meade TG (1967) Life history studies on Cardicola klamathensis (Wales, 1958)

Meade, Pratt (1965) (Trematoda: Sanguinicolidae). Proceedings of the Helminthological Society of Washington 34 (2): 210-212.

Nasir P (1974) British freshwater cercariae. The British Museum, London.

Nithiuthai S, Suwansaksri J,Wiwanitkit V, Chaengphukeaw P (2002) A survey of metacercariae in cyprinoid fish in Nakhon Ratchasima, northeast Thailand. The Southeast Asian Journal of Tropical Medicine and Public Health 33 (3): 103-105.

Olivier LC, Schneiderman M (1956) Method for estimating the density of aquatic snail population. Experimental Parasitology 5: 109-117.

Pace GL (1973) Freshwater snails of Taiwan. Malacological Review 1(suppl): 24-31.

Pinto HA, De Melo AL (2011) A checklist of trematodes (Platyhelminthes) transmitted by Melanoides tuberculata (Mollusca: Thiaridae). Zootaxa 2799: 15-28.

Pointier JP (1989) Conchological studies of Thiara (Melanoides) tuberculata (Mollusca: Gastropoda: Thiaridae) in the French West Indies. Walkerana 3: 203-209.

Pointier JP (1993) The introduction of Melanoides tuberculata (Mollusca: Thiaridae) to the island of Saint Lucia (West Indies) and its role in the decline of Biomphalaria glabrata, the snail intermediate hosts of Schistosoma mansoni. Acta Tropica 54: 13-18.

Pointier JP, Jourdane J (2000) Biological control of the snail hosts of Schistosomiasis in areas of low transmission: the example of the Caribbean area. Acta Tropica 77: 53-60.

Pungpak S, Radomyos P, Radomyos B, Schelp FP, Jongsuksuntigul P, Bunnag D (1998) Treatment of Opisthorchis viverrini and intestinal fluke infections with praziquentel. The Southeast Asian Journal of Tropical Medicine and Public Health 29: 246-9.

Radomyos B, Wongsaroj T, Wilairatana P, Radomyos P, Praevanich R, Meesomboon V, Jongsuksuntikul P (1998) Opisthorchiasis and intestinal fluke infections in northern Thailand. The Southeast Asian Journal of Tropical Medicine and Public Health 29: 123-127.

Samadi S, Mavárez J, Pointier JP, Delay B, Jarne P (1999) Microsatellite and morphological analysis of population structure in the parthenogenetic freshwater snail Melanoides tuberculata: insights into creation of clonal variability. Molecular Ecology 8: 1141-1153.

Schell SC (1962) Parasitology laboratory manual. Wiley. New York.

Schell SC (1970) How to know the Trematode. W. C. Brown Publishers, Iowa.

Smith SJ, Hickman JL (1983) Two strigeoid trematodes, Apatemon (Apatemon) gracilis (Rudolphi, 1819) and Diploetomum (Dolichorchis) galaxiae n. sp., which encyst in the freshwater fish Galaxias auratus Johnston in Lake Crescent, Tasmania. Papers and Proceedings of the Royal Society of Tasmania 117: 21-39.

Sri-aroon P, Lohachit C, Harada M (2005) Brackish-water Mollusks of Surat Thani Province, Southern Thailand. The Southeast Asian Journal of Tropical Medicine and Public Health 36 (suppl 4): 180-188.

Sripa B, Kaewkes S, Intapan PM, Maleewong W, Brindley PJ (2010) Food-borne trematodiases in Southeast Asia: epidemiology, pathology, clinical manifestation and control. Advances in Parasitology 72: 305-50.

Srisawangwong T, Sithithaworn P, Tesana S (1997b) Metacercariae isolated from cyprinoid fishes in Khon Kaen district by digestion technic. The Southeast Asian Journal of Tropical Medicine and Public Health 28 (suppl 1): 224-226.

Strong EE, Gargominy O, Ponder WF, Bouchet P (2008) Global diversity of gastropods (Gastropoda; Mollusca) in freshwater. Hydrobiologia 595: 149-166.

Strong EE, Colgan DJ, Healy JM, Lydeard C, Ponder WF, Glaubrecht M (2011) Phylogeny of the gastropod superfamily Cerithioidea using morphology and molecules. Zoological Journal of the Linnean Society 162: 43-89.

Sukontason K, Piangjai S, Muangyimpong Y, Sukontason K, Methanitikorn R, Chaithong U (1999) Prevalence of trematode metacercariae in cyprinoid fish of Ban Pao district, Chiang Mai Province, northern Thailand. The Southeast Asian Journal of Tropical Medicine and Public Health 30: 365-70.

Sukontason K, Unpunyo P, Sukontason KL, Piangjai S (2005) Evidence of Haplorchis taichui infection as pathogenic parasite: Three case reports. Case report. Scandinavian Journal of Infection Diseases 37: 388-390.

Supian Z, Ikhwanuddin AM (2002) Population dynamics of freshwater mollusks (Gastropod: Melanoides tuberculata) in Crocker Range Park, Sabah. Asean Review of Biodiversity and Environmental Conservation. http://www.Arbec.com.my/pdf/art13julysep02.pdf

Tubangui MA, Cabrera BD, Yogore MG (1950) Studies on the human lung fluk (Paragonimus) in the Philippines. A preliminary report. Acta Medica Philippina 6: 371.

Tang AA (1940) A comparative study of two types of Paragonimus occurring in Fukien, south China. Clinical Medicine Journal (Suppl 3): 267.

Temcharoen P (1992a) Malacological survey in the Bhumibol Reservoir, the first dam in Thailand. The Southeast Asian Journal of Tropical Medicine and Public Health 23: 103-110. 
Temcharoen P (1992b) Malacological survey in the Sirikit Reservoir, the largest Earth filled dam in Thailand. The Southeast Asian Journal of Tropical Medicine and Public Health 23: 332-335.

Tesana S (2002) Diversity of mollusks in the Lam Ta Khong Reservoir, Nakhon Ratchasima, Thailand. The Southeast Asian Journal of Tropical Medicine and Public Health 33: 733-738.

Ukong S, Krailas D, Dangprasert T, Channgarm P (2007) Studies on the morphology of cercariae obtained from freshwater snails at Erawan Waterfall, Erawan National Park, Thailand. The Southeast Asian Journal of Tropical Medicine and Public Health 38 (2): 302-312.

Upatham ES, Sornmai S, Thirachantra S, Sitaputra P (1980) Field studies on the bionomics of alpha and gamma races of Tricula aperta in the Mekong River at Khemmarat, Ubol Ratchathani Province, Thailand. In: Bruce JI, Sornmani S, Asch HL, Crawford KA (Eds) The Mekong Schistosome. Malacological Review (suppl 2): 239-261.

Upatham ES, Koura M, Ahmed MD, Awad AH (1981) Studies on the transmission of Schistosoma haematobium and the bionomics of $\mathrm{Bu}$ linus (Ph.) abyssinicus in the Somali Democratic Republic. Annals of Tropical Medicine and Parasitology 75: 63-69.

Upatham ES, Kruatrachue M, Chitramwong Y, Jantataemae S (1995) Malacology. Suksopa Publishing, Bangkok.
Waikagul J, Dekumyoy P, Yoonuan T, Praevanit R (2006) The American Society of Tropical Medicine and Hygiene 74: 848-849.

Watthanakulpanich D, Waikagul J, Maipanich W, Nuamtanong S, Sanguankiat S, Pubampen S, Praevanit R, Mongkhonmu S, Nawa Y (2010) Haplorchis taichui as a possible etiologic agent of irritable bowel syndrome-like symptoms. The Korean Journal of Parasitology 48 (3): 225-229.

Wegeberg AM, Montaudouin XD, Jensen KT (1999) Effect of intermediate host size (Cerastoderma edule) on infectivity of cercariae of three Himasthla species (Echinostomatidae, Trematoda). Journal of Experimental Marine Biology and Ecology 238: 259-269.

Wongratanacheewin S, Pumidonming W, Sermswan RW, Maleewong W (2001) Development of a PCR-based method for the detection of Opisthorchis viverrini in experimentally infected hamsters. Parasitology 122: 175-180.

Urabe M (2005) Cercariae of a species of Philophthalmus detected in a freshwater snail, Semisulcospira libertina, in Japan. Parasitology International 54: 55-7.

Yamaguti S (1975) A synoptical review of life histories of digenetic Trematodes of vertebrates. Keigaku Publishing, Kyoto. 